\title{
DETERMINATION OF MOLECULAR STRUCTURE IN SOLUTION USING VIBRATIONAL CIRCULAR DICHROISM SPECTROSCOPY: THE SUPRAMOLECULAR TETRAMER OF S-2,2'-DIMETHYL-BIPHENYL-6,6'-DICARBOXYLIC ACID
}

\author{
M. Urbanová, V. Setnička, F.J. Devlin and P. J. Stephens*
}

Supporting Information

S1 Contents

S2-S3 Table S1: Harmonic vibrational frequencies and dipole strengths of 2 and $(2)_{2}$.

S4 Table S2: B3LYP Structural parameters of 2 and $(\mathbf{2})_{2}$.

S5-S13 Table S3: B3LYP/6-31G* harmonic vibrational frequencies, dipole strengths and rotational strengths of the six conformations of $(\mathrm{S}-\mathbf{1})_{4}$.

S14 Table S4: B3LYP/6-31G* structural parameters of conformations aaaa, aaab and bbbb of $(\mathrm{S}-1)_{4}$.

S15 Fig. S1: Basis set dependence of the calculated IR spectrum of $(2)_{2}$.

S16 Fig. S2: B3LYP/6-31G* IR spectra of the six conformations of $(\mathrm{S}-1)_{4}$ and the experimental IR spectrum.

S17 Fig. S3: Comparison of B3LYP/6-31G* frequencies and dipole strengths of conformation aaab of $(\mathrm{S}-1)_{4}$ and the experimental IR spectrum.

S18 Fig. S4: B3LYP/6-31G* IR spectra of the six conformations of $(\mathrm{S}-\mathbf{1})_{4}$ and the experimental IR spectrum.

S19 Fig. S5: Comparison of B3LYP/6-31G* frequencies and dipole strengths of conformation aaab of $(\mathrm{S}-1)_{4}$ and the experimental IR spectrum.

S20 Fig. S6: B3LYP/6-31G* VCD spectra of the six conformations of $(\mathrm{S}-1)_{4}$ and the experimental VCD spectrum.

S21 Fig. S7: Comparison of B3LYP/6-31G* frequencies and rotational strengths of conformation aaab of (S-1) 4 and the experimental VCD spectrum.

S22 Fig. S8: B3LYP/6-31G* VCD spectra of the six conformations of $(\mathrm{S}-\mathbf{1})_{4}$ and the experimental VCD spectrum.

S23 Fig. S9: Comparison of B3LYP/6-31G* frequencies and rotational strengths of conformation aaab of $(\mathrm{S}-\mathbf{1})_{4}$ and the experimental VCD spectrum.

S24 Fig. S10: The B3LYP/6-31G* structure of the aaab conformer of $(\mathrm{S}-\mathbf{1})_{4}$ and the Xray structure of $(\mathrm{R}-\mathbf{1})_{4}$.

S25-S39 Cartesian coordinates of the six conformations of $(\mathrm{S}-\mathbf{1})_{4}$.

S40 Methods.

S41 References. 
Table S1: Harmonic vibrational frequencies and dipole strengths of $\mathbf{2}$ and $(\mathbf{2})_{2}{ }^{\mathrm{a}}$.

2

\begin{tabular}{|c|c|c|c|c|c|c|c|c|c|}
\hline \multicolumn{3}{|c|}{ B3LYP/6-31G* } & \multicolumn{3}{|c|}{ B3LYP/6-31G* } & \multicolumn{2}{|c|}{ B3LYP/6-311G*** } & \multicolumn{2}{|c|}{ B3LYP/6-311++G** } \\
\hline mode & $v$ & $\mathrm{D}$ & mode & $v$ & $\mathrm{D}$ & $v$ & $\mathrm{D}$ & v & $\mathrm{D}$ \\
\hline 39 & 3700 & 77.1 & 84 & 3235 & 0 & 3211 & 0 & 3210 & 0 \\
\hline 38 & 3234 & 3.9 & 83 & 3235 & 5.3 & 3211 & 4.3 & 3210 & 3.6 \\
\hline 37 & 3228 & 6.3 & 82 & 3229 & 9.3 & 3205 & 47.8 & 3204 & 136.9 \\
\hline 36 & 3210 & 27.0 & 81 & 3229 & 0 & 3205 & 0 & 3204 & 0 \\
\hline 35 & 3200 & 19.5 & 80 & 3210 & 7.9 & 3191 & 3377.5 & 3195 & 6032.2 \\
\hline 34 & 3188 & 0.6 & 79 & 3210 & 0 & 3189 & 0 & 3188 & 0 \\
\hline 33 & 1821 & 653.0 & 78 & 3200 & 39.5 & 3186 & 4013.0 & 3188 & 1055.3 \\
\hline 32 & 1663 & 42.1 & 77 & 3200 & 0 & 3179 & 36.0 & 3179 & 0 \\
\hline 31 & 1642 & 11.2 & 76 & 3188 & 1591.1 & 3179 & 0 & 3179 & 28.8 \\
\hline 30 & 1543 & 4.0 & 75 & 3188 & 0 & 3166 & 0 & 3166 & 0 \\
\hline 29 & 1498 & 43.8 & 74 & 3185 & 5450.4 & 3166 & 12.4 & 3166 & 5.4 \\
\hline 28 & 1394 & 341.0 & 73 & 3088 & 0 & 3087 & 0 & 3101 & 0 \\
\hline 27 & 1368 & 13.2 & 72 & 1766 & 1918.5 & 1746 & 2080.6 & 1731 & 2326.6 \\
\hline 26 & 1353 & 11.9 & 71 & 1721 & 0 & 1697 & 0 & 1685 & 0 \\
\hline 25 & 1224 & 421.2 & 70 & 1664 & 0 & 1646 & 0 & 1642 & 0 \\
\hline 24 & 1202 & 448.1 & 69 & 1664 & 129.3 & 1646 & 129.7 & 1642 & 145.2 \\
\hline 23 & 1195 & 2.8 & 68 & 1643 & 0 & 1625 & 0 & 1621 & 0 \\
\hline 22 & 1128 & 163.2 & 67 & 1642 & 93.9 & 1624 & 113.4 & 1620 & 130.1 \\
\hline 21 & 1104 & 225.2 & 66 & 1545 & 0 & 1526 & 0 & 1524 & 36.2 \\
\hline 20 & 1054 & 42.1 & 65 & 1545 & 47.4 & 1526 & 36.8 & 1523 & 0 \\
\hline 19 & 1020 & 3.5 & 64 & 1512 & 0 & 1500 & 0 & 1486 & 0 \\
\hline 18 & 1010 & 0.4 & 63 & 1499 & 177.1 & 1483 & 195.4 & 1480 & 155.7 \\
\hline 17 & 985 & 0.5 & 62 & 1495 & 0 & 1481 & 0 & 1474 & 0 \\
\hline 16 & 958 & 5.5 & 61 & 1474 & 703.4 & 1466 & 461.8 & 1453 & 521.7 \\
\hline 15 & 869 & 0.3 & 60 & 1371 & 66.9 & 1350 & 36.5 & 1350 & 34.2 \\
\hline 14 & 818 & 0.2 & 59 & 1370 & 0 & 1350 & 0 & 1350 & 0 \\
\hline 13 & 778 & 51.4 & 58 & 1359 & 716.0 & 1349 & 1254.8 & 1346 & 853.4 \\
\hline 12 & 731 & 706.3 & 57 & 1358 & 0 & 1346 & 0 & 1344 & 0 \\
\hline 11 & 705 & 9.3 & 56 & 1331 & 1670.9 & 1323 & 1512.9 & 1317 & 2013.4 \\
\hline 10 & 637 & 325.2 & 55 & 1326 & 0 & 1317 & 0 & 1309 & 0 \\
\hline 9 & 632 & 4.9 & 54 & 1211 & 0 & 1199 & 0 & 1199 & 0 \\
\hline 8 & 614 & 417.4 & 53 & 1210 & 183.9 & 1198 & 189.9 & 1198 & 212.1 \\
\hline 7 & 497 & 46.2 & 52 & 1195 & 0 & 1184 & 0 & 1184 & 0 \\
\hline 6 & 437 & 59.9 & 51 & 1195 & 2.2 & 1184 & 3.1 & 1184 & 4.5 \\
\hline 5 & 416 & 4.1 & 50 & 1162 & 0 & 1153 & 0 & 1150 & 0 \\
\hline 4 & 384 & 56.8 & 49 & 1158 & 34.7 & 1150 & 41.0 & 1147 & 65.7 \\
\hline 3 & 216 & 25.2 & 48 & 1111 & 59.3 & 1101 & 80.6 & 1101 & 87.9 \\
\hline 2 & 160 & 11.2 & 47 & 1111 & 0 & 1101 & 0 & 1101 & 0 \\
\hline \multirow[t]{2}{*}{1} & 69 & 52.0 & 46 & 1055 & 0 & 1047 & 0 & 1046 & 0 \\
\hline & & & 45 & 1055 & 60.0 & 1047 & 85.3 & 1046 & 98.5 \\
\hline
\end{tabular}




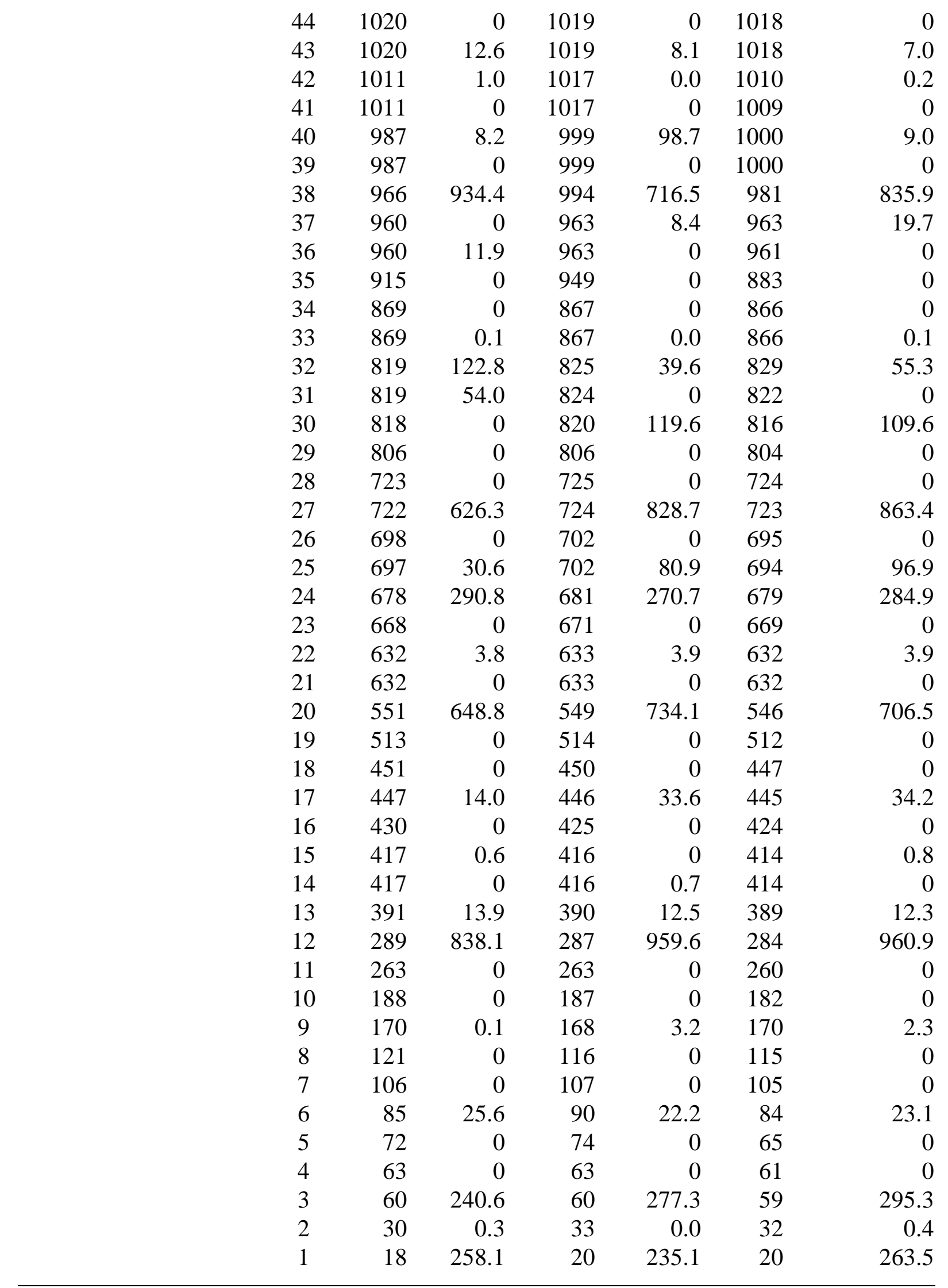

${ }^{\mathrm{a}} \mathrm{v}$ in $\mathrm{cm}^{-1}, \mathrm{D}$ in $10^{-40} \mathrm{esu}^{2} \mathrm{~cm}^{2}$. 
Table S2: B3LYP Structural parameters of 2 and $(\mathbf{2})_{2}{ }^{\mathrm{a}}$.

Bond Lengths

\begin{tabular}{|c|c|c|c|c|}
\hline & \multirow{2}{*}{$\begin{array}{c}2 \\
6-31 \mathrm{G}^{*}\end{array}$} & \multicolumn{3}{|c|}{$(\mathbf{2})_{2}$} \\
\hline & & $6-31 \mathrm{G}^{*}$ & $6-311 \mathrm{G}^{* *}$ & $6-311++\mathrm{G}^{* *}$ \\
\hline $\mathrm{C} 1 \mathrm{O} 2$ & 1.215 & 1.235 & 1.229 & 1.230 \\
\hline $\mathrm{O} 2 \mathrm{H} 3$ & & 1.673 & 1.653 & 1.662 \\
\hline $\mathrm{O} 2 \ldots \mathrm{O} 4$ & & 2.677 & 2.653 & 2.662 \\
\hline H3O4 & 0.975 & 1.005 & 1.000 & 1.000 \\
\hline O4C5 & 1.359 & 1.324 & 1.320 & 1.323 \\
\hline \multirow[t]{4}{*}{ C1C9 } & 1.487 & 1.486 & 1.487 & 1.486 \\
\hline & \multicolumn{4}{|c|}{ Bond Angles } \\
\hline & 2 & & $(\mathbf{2})_{2}$ & \\
\hline & $6-31 G^{*}$ & $6-31 G^{*}$ & $6-311 \mathrm{G}^{* *}$ & $6-311++\mathrm{G}^{* *}$ \\
\hline $\mathrm{C} 1 \mathrm{O} 2 \mathrm{H} 3$ & & 125.0 & 126.2 & 126.8 \\
\hline $\mathrm{O} 2 \mathrm{H} 3 \mathrm{O} 4$ & & 178.6 & 180.0 & 179.6 \\
\hline H3O4C5 & 105.5 & 109.9 & 110.2 & 110.3 \\
\hline O4C5O6 & 121.8 & 123.6 & 123.5 & 123.3 \\
\hline
\end{tabular}

${ }^{a}$ Both molecules are planar. (2) has $\mathrm{C}_{2 \mathrm{~h}}$ symmetry. Bond lengths in $\AA$, bond angles in degrees. Atom numbering for $\mathbf{2}$ is the same as for $(\mathbf{2})_{2}$. 
Table S3: B3LYP/6-31G* harmonic vibrational frequencies, dipole strengths and rotational strengths of the six conformations of $(\mathrm{S}-\mathbf{1})_{4}{ }^{\mathrm{a}}$.

\begin{tabular}{|c|c|c|c|c|c|c|c|c|c|c|c|c|c|c|c|c|c|}
\hline \multirow[b]{2}{*}{$v$} & \multirow{2}{*}{$\frac{\text { aaaa }}{\mathrm{D}}$} & \multirow[b]{2}{*}{$\mathrm{R}$} & \multirow[b]{2}{*}{$v$} & \multirow{2}{*}{$\frac{\mathbf{a a a b}}{\mathrm{D}}$} & \multirow[b]{2}{*}{$\mathrm{R}$} & & \multirow{2}{*}{$\frac{\mathbf{a} \mathbf{a b b}}{\mathrm{D}}$} & \multirow[b]{2}{*}{$\mathrm{R}$} & \multirow[b]{2}{*}{$v$} & \multirow{2}{*}{$\frac{\mathbf{a b a b}}{\mathrm{D}}$} & \multirow[b]{2}{*}{$\mathrm{R}$} & & \multirow{2}{*}{$\frac{\mathbf{a b b b}}{\mathrm{D}}$} & \multirow[b]{2}{*}{$\mathrm{R}$} & \multirow[b]{2}{*}{$v$} & \multirow{2}{*}{$\frac{\text { bbbb }}{\mathrm{D}}$} & \multirow[b]{2}{*}{$\mathrm{R}$} \\
\hline & & & & & & & & & & & & & & & & & \\
\hline 3245 & 2 & -8 & 3245 & 4 & -2 & 3245 & 2 & 0 & 3244 & 0 & -3 & 3244 & 0 & -2 & 3238 & 0 & 0 \\
\hline 3245 & 2 & -8 & 3245 & 0 & 3 & 3245 & 3 & -4 & 3244 & 0 & 0 & 3244 & 7 & 3 & 3238 & 1 & 1 \\
\hline 3245 & 0 & 0 & 3245 & 1 & -14 & 3245 & 7 & 5 & 3244 & 14 & 4 & 3239 & 10 & -1 & 3238 & 1 & 1 \\
\hline 3245 & 0 & 0 & 3245 & 10 & 11 & 3245 & 4 & 2 & 3244 & 0 & -1 & 3239 & 0 & 6 & 3238 & 0 & 0 \\
\hline 3245 & 0 & 0 & 3245 & 9 & 1 & 3238 & 2 & 1 & 3239 & 5 & -27 & 3239 & 0 & -2 & 3238 & 0 & 0 \\
\hline 3245 & 0 & -10 & 3245 & 0 & 2 & 3238 & 5 & -1 & 3239 & 17 & 25 & 3239 & 6 & 6 & 3238 & U & 5 \\
\hline 3245 & 0 & -10 & 3239 & 2 & -13 & 3238 & 6 & 2 & 3239 & 0 & 0 & 3238 & 10 & -3 & 3238 & 0 & 5 \\
\hline 3245 & 30 & 35 & 3239 & 9 & 16 & 3238 & 7 & 3 & 3239 & 1 & 14 & 3238 & 0 & 6 & 3238 & & 2 \\
\hline 3214 & 0 & 0 & 3214 & 1705 & -2029 & 3213 & 3585 & -4682 & 3208 & 52 & 1954 & 3209 & 4104 & -2401 & 3204 & & 0 \\
\hline 3206 & 4257 & -4468 & 3208 & 6700 & -7795 & 3205 & 1105 & -426 & 3208 & 7081 & -6902 & 3205 & 46 & 69 & 3204 & 19 & 10 \\
\hline 3206 & 4257 & -4468 & 3205 & 257 & -369 & 3204 & 31 & -51 & 3204 & 17 & -57 & 3204 & 7 & -53 & 3204 & & 10 \\
\hline 3204 & 0 & 0 & 3204 & 18 & -66 & 3204 & 2 & 10 & 3204 & 0 & 0 & 3204 & 5 & -15 & 3204 & 0 & 0 \\
\hline 3204 & . 139 & -310 & 3204 & 107 & 216 & 3204 & 82 & -55 & 3204 & 169 & 24 & 3204 & 107 & -52 & 3204 & 177 & -26 \\
\hline 3204 & 0 & 38 & 3204 & 60 & -11 & 3204 & 78 & 154 & 3204 & 4 & 112 & 3204 & 2 & 62 & 3204 & 0 & 17 \\
\hline 3204 & 0 & 38 & 3204 & 9 & -158 & 3204 & 6 & -10 & 3204 & 7 & -155 & 3204 & 79 & -102 & 3204 & 0 & 17 \\
\hline 3204 & 0 & 0 & 3204 & 0 & -19 & 3204 & 4 & & 3204 & 0 & 0 & & 2 & & 3204 & & 0 \\
\hline 3203 & 0 & 0 & 3203 & 285 & -473 & 3203 & 669 & -1087 & 3202 & 5256 & -6658 & 3202 & 1820 & -1233 & 3181 & 0 & 0 \\
\hline 3200 & 7913 & 10800 & 3202 & 5321 & -7778 & 3198 & 8571 & -2200 & 3201 & 104 & 2571 & 3182 & 17 & 15 & 3181 & 5 & 11 \\
\hline 3200 & 7913 & & 3193 & & 6797 & 3181 & 68 & -32 & 3182 & 29 & 21 & & & 13 & 81 & & 11 \\
\hline 3181 & 1081 & 20180 & 3182 & 34 & -34 & 3181 & 3 & 52 & 3182 & 0 & 0 & 3182 & 7 & 8 & 3181 & 0 & 0 \\
\hline 3181 & 0 & 0 & 3182 & 12 & 154 & 3181 & 60 & -32 & 3182 & 73 & -62 & 3182 & & & 181 & & -45 \\
\hline 3181 & 107 & -179 & 3181 & 15 & -11 & 3181 & 7 & 67 & 3182 & 4 & 106 & 3182 & 48 & 80 & 3181 & 72 & -45 \\
\hline 3181 & 107 & -179 & 3181 & 60 & -62 & 3181 & 16 & -26 & 3181 & 0 & 0 & 3182 & 24 & -22 & 3181 & 0 & 0 \\
\hline 3181 & 0 & 0 & 3181 & 38 & -88 & 3181 & 25 & -29 & 3181 & 40 & -46 & 3181 & 20 & -25 & 181 & 9 & 173 \\
\hline 3180 & 0 & 0 & 3181 & 24 & -56 & 3180 & 25 & -38 & 3181 & 3 & 38 & 3181 & 3 & 2 & 3177 & 0 & 0 \\
\hline 3180 & 0 & 6 & 3181 & 1 & -8 & 3180 & 8 & -6 & 3181 & 16 & -59 & 3175 & 6053 & -7318 & 3166 & & \\
\hline 3180 & 0 & 6 & 3181 & 1 & 13 & 3171 & 8224 & 10622 & 3166 & 12582 & 21715 & 43164 & 127 & 17129 & 93166 & 129 & 16112 \\
\hline 3180 & 674 & 12460 & 3158 & 5133 & 13424 & +3153 & 4484 & 20966 & 3154 & 1599 & 28335 & 53148 & 2213 & 30280 & ) 3144 & 1771 & 34395 \\
\hline 3130 & 0 & 0 & 3130 & 25 & -133 & 3130 & 33 & -20 & 3130 & 0 & 0 & 3130 & 17 & 156 & 3130 & 0 & 0 \\
\hline 3130 & 32 & -312 & 3130 & 41 & 167 & 3130 & 36 & -47 & 3130 & 55 & -258 & 3130 & 44 & -241 & 3129 & 55 & -269 \\
\hline 3130 & 32 & -312 & 3130 & 0 & -20 & 3130 & 27 & -41 & 3130 & 44 & 406 & 3130 & 41 & -96 & 3129 & 55 & -269 \\
\hline 3130 & 0 & 0 & 3130 & 59 & -143 & 3129 & 28 & 76 & 3130 & 25 & -99 & 3130 & 43 & 173 & 3129 & 0 & 0 \\
\hline 3130 & 96 & 118 & 3130 & 30 & -7 & 3129 & 37 & -8 & 3128 & 0 & 0 & 3130 & 21 & 253 & 3129 & 0 & 0 \\
\hline 3130 & 29 & 273 & 3130 & 24 & 141 & 3129 & 32 & 26 & 3128 & 47 & -275 & 3130 & 32 & -250 & 3129 & 41 & 427 \\
\hline 3130 & 29 & 273 & 3129 & 5 & 114 & 3129 & 24 & 1 & 3128 & 57 & 13 & 3129 & 25 & -137 & 3129 & 41 & 427 \\
\hline 3130 & 0 & 0 & 3129 & 50 & -89 & 3129 & 29 & 24 & 3128 & 10 & 217 & 3129 & 31 & 100 & 3129 & 48 & -455 \\
\hline 3111 & 0 & 0 & 3114 & 3 & -223 & 3115 & 10 & -81 & 3114 & 0 & 0 & 3117 & 10 & -422 & 3111 & 0 & 0 \\
\hline 3111 & 4 & -344 & 3113 & 23 & -2 & 3115 & 22 & -408 & 3114 & 15 & -742 & 3110 & 36 & -47 & 3111 & 17 & -64 \\
\hline 3111 & 4 & -344 & 3113 & 9 & -449 & 3111 & 15 & 3 & 3110 & 0 & 0 & 3110 & 3 & 64 & 3111 & 17 & -64 \\
\hline 3111 & 0 & 0 & 3110 & 11 & -46 & 3110 & 6 & -9 & 3110 & 1 & 29 & 3110 & 1 & 7 & 3110 & 4 & 197 \\
\hline 3110 & 2 & 112 & 3110 & 6 & 9 & 3110 & 17 & 8 & 3110 & 61 & -59 & 3110 & 37 & 12 & 3110 & 0 & 0 \\
\hline
\end{tabular}


$\begin{array}{llllllll}3110 & 26 & -317 & 3110 & 0 & -1 & 3110 & 16\end{array}$ $\begin{array}{llllllll}3110 & 26 & -317 & 3110 & 27 & -2 & 3110 & 29\end{array}$ $\begin{array}{llllllll}3110 & 0 & 0 & 3110 & 21 & -22 & 3110 & 25\end{array}$ $\begin{array}{llllllll}3110 & 0 & 0 & 3110 & 18 & 57 & 3110 & 22\end{array}$ $\begin{array}{llllllll}3110 & 43 & 204 & 3110 & 40 & 42 & 3109 & 20\end{array}$ $\begin{array}{llllllll}3110 & 43 & 204 & 3110 & 27 & 27 & 3070 & 5\end{array}$ $\begin{array}{llllllll}3110 & 0 & 0 & 3069 & 2 & -198 & 3069 & 36\end{array}$ $\begin{array}{llllllll}3054 & 0 & 0 & 3054 & 52 & 131 & 3054 & 45\end{array}$ $\begin{array}{llllllll}3054 & 59 & 337 & 3054 & 14 & -2 & 3054 & 35\end{array}$ $\begin{array}{lllll}3054 & 59 & 337 & 3054 & 30\end{array}$ $\begin{array}{llllll}3054 & 23 & -379 & 3054 & 45\end{array}$ $\begin{array}{lllll}3053 & 0 & 0 & 3053 & 17\end{array}$ $\begin{array}{lll}172 & 3054 & 24\end{array}$ $\begin{array}{lll}-80 & 3054 & 37\end{array}$

$17 \quad 3053 \quad 16$ $\begin{array}{llllllll}3053 & 67 & -183 & 3053 & 26 & -167 & 3053 & 60\end{array}$ $\begin{array}{llllllll}3053 & 67 & -183 & 3053 & 60 & -132 & 3053 & 33\end{array}$ $\begin{array}{llllllll}3053 & 0 & 0 & 3053 & 37 & -6 & 3053 & 39\end{array}$ $\begin{array}{lllllllll}1771 & 4541 & 4921 & 1768 & 3875 & 2001 & 1767 & 3044 & -259\end{array}$ $\begin{array}{llllllllll}1761 & 398 & -2722 & 1761 & 383 & -2705 & 1758 & 5 & -242 & 176\end{array}$ $\begin{array}{lllllllllllllllllll}1761 & 398 & -2722 & 1756 & 85 & -253 & 1753 & 2089 & 975 & 1752 & 3935 & 3657 & 1753 & 3883 & 3882 & 1753 & 3801 & 4092\end{array}$ $\begin{array}{llllllllllllllllll}1754 & 0 & 0 & 1750 & 1917 & 745 & 1749 & 2004 & -601 & 1749 & 77 & -1568 & 1748 & 1086 & -3910 & 1747 & 1268 & -8744\end{array}$ 17220

$1719 \quad 4 \quad 71$

17194

$1718 \quad 0$

$0 \quad 1722 \quad 0$

$\begin{array}{lll}71 & 1720 & 3\end{array}$

$1650 \quad 0$

$1650 \quad 6$

$1650 \quad 6$

$1650 \quad 0$

$1648 \quad 0$

$\begin{array}{lll}71 & 1718 & 2\end{array}$

$\begin{array}{lll}9 & 1722 & 3\end{array}$

$\begin{array}{lll}17 & 1719 & 1\end{array}$

$1648 \quad 12$

$\begin{array}{lllll}1648 & 12 & 29 & 1648 & 73\end{array}$

$\begin{array}{lllll}1648 & 13 & -56 & 1648 & 29\end{array}$

$1631 \quad 0$

$1630 \quad 61$

163061

$1630 \quad 0$

16290

$\begin{array}{llll}0 & 1631 & 7\end{array}$

$\begin{array}{lll}-134 & 1630 & 23\end{array}$

$\begin{array}{lll}26 & 1706 \quad 3\end{array}$

$15 \quad 1705 \quad 0$

$\begin{array}{lll}2 & 1650 & 9\end{array}$

$\begin{array}{lll}6 & 1720\end{array}$

$\begin{array}{lll}34 & 1720 & 0\end{array}$

$\begin{array}{lll}86 & 1705 & 0\end{array}$

$27 \quad 1704 \quad 0$

$\begin{array}{ccc}8 & 1650 & 0\end{array}$

$\begin{array}{lll}2 & 1650 & 8\end{array}$

$\begin{array}{lll}2 & 1650 & 2\end{array}$

$\begin{array}{ccc}1 & 1650 & 13\end{array}$

$\begin{array}{llll}-8 & 1650 & 46 & -18\end{array}$

1650

$\begin{array}{llll}-1 & 1649 & 126 & -7\end{array}$

$\begin{array}{llll}4 & 1648 & 72\end{array}$

$\begin{array}{lll}33 & 1648 & 75\end{array}$

$\begin{array}{lll}62 & 1648 \quad 96\end{array}$

$\begin{array}{lll}-91 & 1648 & 5\end{array}$

$\begin{array}{lll}-22 & 1631 \\ -53 & 3630\end{array}$

$\begin{array}{lll}-53 & 1630 & 36\end{array}$

$\begin{array}{lll}-154 & 1630 & 83\end{array}$

$\begin{array}{ccc}-134 & 1630 & 99 \\ 0 & 1630 & 29\end{array}$

$\begin{array}{lll}21 & 1629 & 6\end{array}$

$\begin{array}{llllllll}1629 & 150 & -151 & 1628 & 108 & 171 & 1628 & 38\end{array}$

$\begin{array}{llllllll}1629 & 150 & -151 & 1628 & 34 & 105 & 1628 & 18\end{array}$

$\begin{array}{lllll}1628 & 109 & 501 & 1627 & 15\end{array}$

$\begin{array}{lllll}1533 & 0 & 0 & 1532 & 5\end{array}$

$\begin{array}{lllll}1533 & 11 & -30 & 1532 & 8\end{array}$

$\begin{array}{lllll}1533 & 11 & -30 & 1532 & 7\end{array}$

15330

$1530 \quad 0$

$\begin{array}{lll}0 & 1532 & 7\end{array}$

$1530 \quad 0$

$\begin{array}{lll}0 & 1529 & 0\end{array}$

$\begin{array}{lll}-30 & 1628 & 4\end{array}$

$\begin{array}{lll}-18 & 1533 & 5\end{array}$

$\begin{array}{lll}-20 & 1533 & 4\end{array}$

$\begin{array}{lll}30 & 1533 & 13\end{array}$

$\begin{array}{ll}-21 & 1532\end{array}$

$\begin{array}{llll}8 & 1530 & 11\end{array}$

71

$\begin{array}{lll}1648 & 0\end{array}$

$\begin{array}{lll}58 & 1648 & 10\end{array}$

$-24$

$1648 \quad 177$

$\begin{array}{llll}-25 & 1648 & 3\end{array}$

$\begin{array}{ll}-15 & 1630\end{array}$

$\begin{array}{ll}-30 & 1630\end{array}$

$\begin{array}{lll}-96 & 1629 & 191\end{array}$

$\begin{array}{lll}-13 & 1629 & 2\end{array}$

$\begin{array}{ll}65 & 1627\end{array}$

$\begin{array}{lll}70 & 1627 & 40\end{array}$

$\begin{array}{ll}0 & 1721\end{array}$

$\begin{array}{ll}-35 & 1708\end{array}$

$\begin{array}{ll}0 & 1705\end{array}$

$4 \quad 1705$

$\begin{array}{ll}0 & 1650\end{array}$

$\begin{array}{lll}-14 & 1649 & 30\end{array}$

$\begin{array}{lll}-98 & 1649 & 215\end{array}$

1001649

$\begin{array}{lll}0 & 1648 & 57\end{array}$

$\begin{array}{lll}44 & 1648 & 158\end{array}$

$\begin{array}{lll}16 & 1648 & 135\end{array}$

$\begin{array}{ll}-60 & 1648\end{array}$

$\begin{array}{ll}-11 & 1630\end{array}$

$\begin{array}{lll}0 & 1630 \quad 95\end{array}$

$\begin{array}{ll}-222 & 1629\end{array}$

\begin{tabular}{ll}
$157 \quad 1629$ \\
\hline
\end{tabular}

$\begin{array}{ll}-9 & 1627\end{array}$

$\begin{array}{lll}107 & 1627 & 46\end{array}$

$\begin{array}{llll}0 & 1627 & 7\end{array}$

$\begin{array}{lll}-9 & 1627 & 22\end{array}$

$\begin{array}{lll}-22 & 1532 & 0\end{array}$

$-43$

$\begin{array}{lll}24 & 1532 & 10\end{array}$

$\begin{array}{lll}-3 & 1532 & 3\end{array}$

$\begin{array}{lll}-2 & 1532 & 20\end{array}$

$\begin{array}{lll}-7 & 1528 & 0\end{array}$

$\begin{array}{lll}-4 & 1528 & 2\end{array}$

$1530 \quad 0$

$\begin{array}{lll}3 & 1529 & 40 \\ 3 & 1528 & 0\end{array}$

$\begin{array}{lll}-17 & 1529 & 33\end{array}$

$\begin{array}{lllll}1529 & 94 & -19 & 1528 & 6\end{array}$ $\begin{array}{llll}0 & 1529 & 20\end{array}$

$\begin{array}{lll}-3 & 1528 & 41\end{array}$ $\begin{array}{ll}-7 & 1528\end{array}$

$\begin{array}{lll}18 & 1528 & 98\end{array}$ $\begin{array}{lll}0 & 1532\end{array}$

$\begin{array}{lll}57 & 1532 & 13\end{array}$

$\begin{array}{lll}-33 & 1532\end{array}$

$\begin{array}{ll}-27 & 1532\end{array}$

$\begin{array}{lll}0 & 1529\end{array}$

$\begin{array}{lll}-35 & 1528 & 53\end{array}$

$\begin{array}{lll}-12 & 1528 & 4\end{array}$

$\begin{array}{lll}47 & 1528 & 67\end{array}$ $\begin{array}{lll}25 & 3110 & 62\end{array}$

$\begin{array}{llll}-3 & 3110 & 62 & 11\end{array}$

$\begin{array}{llll}-45 & 3110 & 0 & 0\end{array}$

$\begin{array}{llll}62 & 3070 & 0 & 0\end{array}$

$\begin{array}{llll}-241 & 3069 & 0 & 0\end{array}$

$\begin{array}{llll}-100 & 3069 & 7 & -494\end{array}$

$\begin{array}{llll}-324 & 3069 & 7 & -494\end{array}$

$\begin{array}{llll}41 & 3054 & 0 & 0\end{array}$

$\begin{array}{llll}29 & 3054 & 66 & 355\end{array}$

$\begin{array}{llll}141 & 3054 & 66 & 355\end{array}$

$\begin{array}{llll}-22 & 3054 & 17 & -372\end{array}$

$\begin{array}{llll}-67 & 3054 & 0 & 0\end{array}$

$\begin{array}{llll}-113 & 3054 & 77 & -201\end{array}$

$\begin{array}{llll}21 & 3054 & 77 & -201\end{array}$

$\begin{array}{llll}-97 & 3054 & 0 & 0\end{array}$

0 


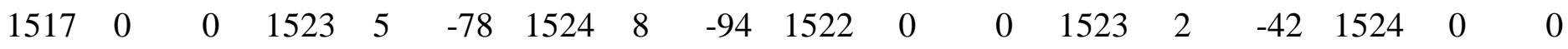

$\begin{array}{llllllllllllllllll}1517 & 56 & -37 & 1516 & 7 & 21 & 1523 & 3 & -60 & 1522 & 15 & -184 & 1522 & 11 & -148 & 1524 & 8 & -155\end{array}$

$\begin{array}{llllllllllllllllll}1517 & 56 & -37 & 1516 & 34 & -23 & 1518 & 3 & 5 & 1517 & 7 & 3 & 1522 & 1 & -33 & 1524 & 8 & -155\end{array}$

$\begin{array}{llllllllllllllllll}1517 & 0 & 0 & 1516 & 19 & -16 & 1517 & 27 & -17 & 1517 & 4 & 18 & 1516 & 1 & -11 & 1523 & 0 & 0\end{array}$

$\begin{array}{llllllllllllllllll}1514 & 0 & 0 & 1514 & 20 & 58 & 1517 & 21 & 70 & 1515 & 0 & 0 & 1516 & 1 & 14 & 1519 & 0 & 0\end{array}$

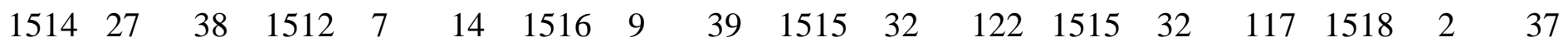

$\begin{array}{llllllllllllllllll}1514 & 27 & 38 & 1512 & 20 & 26 & 1514 & 10 & -27 & 1512 & 1 & 10 & 1514 & 0 & -4 & 1518 & 2 & 37\end{array}$

$\begin{array}{llllllllllllllllll}1514 & 0 & 5 & 1512 & 2 & -16 & 1514 & 14 & 20 & 1512 & 1 & -16 & 1513 & 39 & -2 & 1516 & 48 & 53\end{array}$

$\begin{array}{llllllllllllllllll}1509 & 0 & 0 & 1512 & 17 & -13 & 1514 & 4 & -1 & 1512 & 0 & 0 & 1512 & 0 & -1 & 1515 & 0 & 0\end{array}$

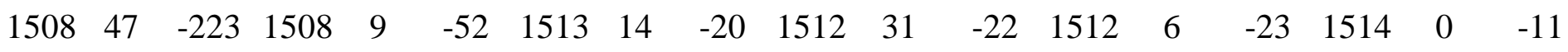

$\begin{array}{llllllllllllllllll}1508 & 47 & -223 & 1507 & 180 & 248 & 1508 & 2 & -30 & 1507 & 123 & 89 & 1511 & 1 & -8 & 1514 & 0 & -11\end{array}$

$\begin{array}{llllllllllllllllll}1505 & 0 & 0 & 1506 & 171 & -395 & 1507 & 395 & 393 & 1507 & 0 & 0 & 1507 & 358 & 842 & 1514 & 0 & 0\end{array}$

$\begin{array}{llllllllllllllllll}1502 & 11 & 30 & 1505 & 26 & -63 & 1506 & 250 & -319 & 1506 & 194 & 673 & 1506 & 423 & -461 & 1508 & 374 & 1356\end{array}$

$\begin{array}{lllllllllllllllllll}1502 & 25 & -41 & 1502 & 4 & 9 & 1506 & 27 & -113 & 1506 & 332 & -777 & 1506 & 138 & -121 & 1507 & 444 & -512\end{array}$

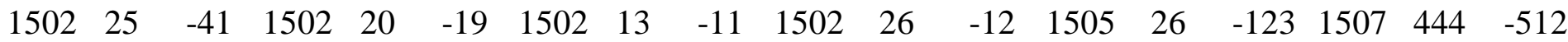

$\begin{array}{llllllllllllllllll}1502 & 0 & 0 & 1502 & 14 & -6 & 1502 & 13 & -1 & 1502 & 3 & 7 & 1502 & 16 & -3 & 1506 & 0 & 0\end{array}$

$\begin{array}{llllllllllllllllll}1501 & 0 & 0 & 1501 & 0 & -1 & 1501 & 0 & 2 & 1501 & 0 & 20 & 1501 & 0 & 15 & 1494 & 0 & 0\end{array}$

$\begin{array}{llllllllllllllllll}1501 & 4 & -58 & 1501 & 1 & -20 & 1501 & 3 & -35 & 1501 & 0 & 0 & 1497 & 196 & 52 & 1494 & 3 & 6\end{array}$

$\begin{array}{llllllllllllllllll}1501 & 4 & -58 & 1501 & 11 & -62 & 1498 & 228 & 38 & 1497 & 435 & 54 & 1494 & 4 & 5 & 1494 & 3 & 6\end{array}$

$\begin{array}{llllllllllllllllll}1501 & 0 & 0 & 1498 & 85 & 57 & 1494 & 2 & 4 & 1497 & 0 & 25 & 1494 & 3 & 5 & 1494 & 0 & 0\end{array}$

$\begin{array}{llllllllllllllllll}1499 & 0 & 0 & 1495 & 360 & 177 & 1494 & 130 & 191 & 1491 & 0 & 0 & 1490 & 0 & -1 & 1491 & 0 & 0\end{array}$

$\begin{array}{llllllllllllllllll}1494 & 398 & 357 & 1491 & 87 & 205 & 1491 & 0 & 3 & 1490 & 4 & 43 & 1490 & 0 & 1 & 1491 & 0 & -1\end{array}$

$\begin{array}{llllllllllllllllll}1494 & 398 & 357 & 1491 & 13 & 14 & 1491 & 2 & 1 & 1490 & 9 & 7 & 1490 & 0 & 0 & 1491 & 0 & -1\end{array}$

$\begin{array}{rlllllllllllllllll}1489 & 0 & 0 & 1489 & 16 & 27 & 1490 & 12 & 43 & 1489 & 0 & 0 & 1490 & 1 & 14 & 1491 & 0 & 0\end{array}$

$\begin{array}{llllllllllllllllll}1477 & 4 & 235 & 1475 & 156 & 286 & 1475 & 317 & 344 & 1474 & 38 & 550 & 1473 & 163 & 885 & 1472 & 253 & 1300\end{array}$

$\begin{array}{llllllllllllllllllll}1473 & 872 & -119 & 1472 & 901 & -138 & 1471 & 667 & -108 & 1471 & 946 & -72 & 1471 & 650 & -740 & 1470 & 692 & -756\end{array}$

$\begin{array}{llllllllllllllllllll}1473 & 872 & -119 & 1471 & 655 & -290 & 1471 & 718 & -420 & 1470 & 594 & -642 & 1470 & 838 & -372 & 1470 & 692 & -756\end{array}$

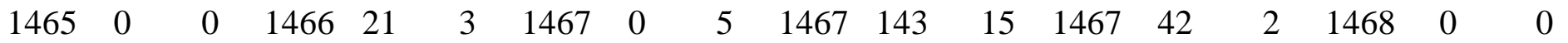

$\begin{array}{llllllllllllllllll}1464 & 0 & 0 & 1463 & 23 & -16 & 1462 & 55 & -52 & 1462 & 0 & 0 & 1461 & 36 & -62 & 1460 & 0 & 0\end{array}$

$\begin{array}{lllllllllllllllllll}1458 & 172 & -182 & 1458 & 195 & -175 & 1459 & 161 & 26 & 1460 & 171 & -295 & 1459 & 112 & -182 & 1459 & 132 & -196\end{array}$

$\begin{array}{llllllllllllllllllll}1458 & 172 & -182 & 1456 & 174 & -159 & 1458 & 157 & -180 & 1456 & 159 & -118 & 1458 & 195 & 196 & 1459 & 132 & -196\end{array}$

$\begin{array}{lllllllllllllllllll}1455 & 86 & 296 & 1454 & 100 & 337 & 1455 & 108 & 281 & 1455 & 108 & 423 & 1455 & 146 & 237 & 1458 & 231 & 741\end{array}$

$\begin{array}{llllllllllllllllll}1449 & 0 & 0 & 1446 & 4 & 4 & 1449 & 1 & 2 & 1447 & 0 & 0 & 1446 & 0 & 1 & 1449 & 0 & 0\end{array}$

$\begin{array}{llllllllllllllllll}1449 & 8 & 10 & 1446 & 1 & 4 & 1449 & 2 & 8 & 1447 & 7 & 9 & 1446 & 4 & 8 & 1449 & 0 & 3\end{array}$

$\begin{array}{llllllllllllllllll}1449 & 8 & 10 & 1445 & 5 & 6 & 1449 & 0 & -2 & 1447 & 1 & 4 & 1446 & 2 & 5 & 1449 & 0 & 3\end{array}$

$\begin{array}{llllllllllllllllll}1449 & 0 & 0 & 1445 & 5 & 5 & 1448 & 4 & 5 & 1447 & 0 & 0 & 1446 & 0 & 0 & 1448 & 0 & 0\end{array}$

$\begin{array}{llllllllllllllllll}1445 & 0 & 0 & 1442 & 0 & 0 & 1445 & 1 & -1 & 1442 & 0 & 0 & 1442 & 1 & -2 & 1445 & 0 & 0\end{array}$

$\begin{array}{llllllllllllllllll}1444 & 0 & 0 & 1442 & 1 & -4 & 1445 & 1 & -2 & 1442 & 3 & -6 & 1442 & 2 & -2 & 1445 & 2 & -7\end{array}$

$\begin{array}{lllllllllllllllllll}1444 & 0 & 0 & 1441 & 0 & 0 & 1444 & 0 & -1 & 1442 & 1 & 4 & 1442 & 1 & -3 & 1445 & 2 & -7\end{array}$

$\begin{array}{rlllllllllllllllll}1444 & 0 & 1 & 1441 & 0 & 0 & 1444 & 1 & -1 & 1441 & 1 & -2 & 1441 & 1 & -1 & 1445 & 5 & 3\end{array}$

$\begin{array}{llllllllllllllllll}1347 & 0 & 0 & 1346 & 572 & -93 & 1346 & 1046 & -168 & 1343 & 2106 & -231 & 1343 & 1477 & -887 & 1340 & 2836 & 7648\end{array}$

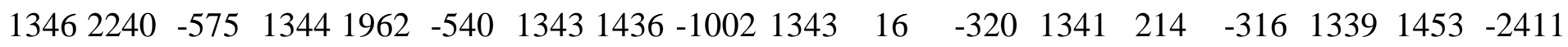

$\begin{array}{llllllllllllllllll}1346 & 2240 & -575 & 1343 & 583 & -107 & 1342 & 50 & 19 & 1341 & 39 & -21 & 1340 & 1851 & 4232 & 1339 & 1453 & -2411\end{array}$

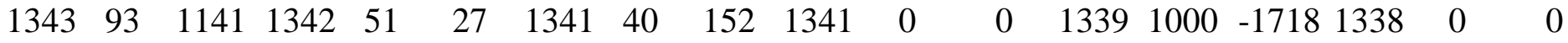

$\begin{array}{rlllllllllllllllll}1342 & 0 & 0 & 1341 & 27 & -11 & 1340 & 1654 & 2124 & 1336 & 1181 & 2746 & 1337 & 495 & 94 & 1337 & 0 & 0\end{array}$

$\begin{array}{lllllllllllllllllll}1342 & 10 & 26 & 1341 & 40 & 196 & 1337 & 813 & -1343 & 1336 & 1769 & -2791 & 1336 & 653 & -1100 & 1335 & 197 & -315\end{array}$

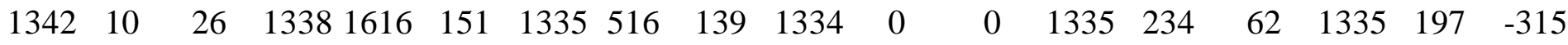


$\begin{array}{llllllllllllllllll}1342 & 0 & 0 & 1335 & 11 & -14 & 1335 & 11 & -15 & 1334 & 42 & -80 & 1335 & 4 & -5 & 1334 & 0 & 0\end{array}$ $\begin{array}{rlllllllllllllllll}1322 & 0 & 0 & 1333 & 12 & -39 & 1333 & 23 & 41 & 1333 & 0 & 0 & 1333 & 4 & 17 & 1333 & 0 & 0\end{array}$

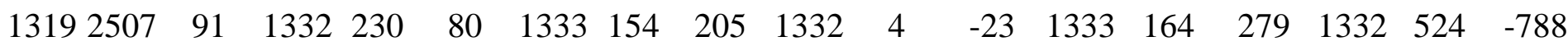

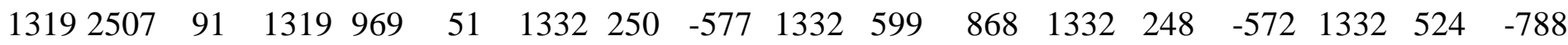
$\begin{array}{llllllllllllllllll}1314 & 0 & 0 & 1317 & 2728 & 603 & 1330 & 106 & 0 & 1332 & 269 & -815 & 1332 & 230 & -341 & 1332 & 0 & 0\end{array}$

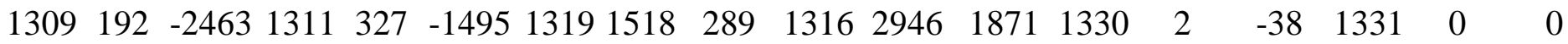
$\begin{array}{llllllllllllllllll}1308 & 505 & 1357 & 1311 & 225 & 447 & 1313 & 1210 & -595 & 1314 & 53 & -1040 & 1330 & 179 & -46 & 1331 & 326 & -77\end{array}$ $\begin{array}{llllllllllllllllll}1308 & 505 & 1357 & 1307 & 394 & 572 & 1311 & 118 & 397 & 1310 & 0 & 0 & 1314 & 1517 & 449 & 1330 & 22 & -202\end{array}$ $\begin{array}{rlllllllllllllllll}1307 & 0 & 0 & 1306 & 177 & 466 & 1307 & 202 & 492 & 1309 & 3 & 87 & 1308 & 0 & 6 & 1330 & 22 & -202\end{array}$ $\begin{array}{llllllllllllllllll}1288 & 0 & 0 & 1288 & 1 & -2 & 1288 & 2 & -6 & 1287 & 1 & -10 & 1287 & 0 & -2 & 1286 & 0 & 0\end{array}$ $\begin{array}{llllllllllllllllll}1288 & 6 & -14 & 1288 & 1 & -6 & 1287 & 6 & -6 & 1287 & 0 & -4 & 1287 & 8 & -5 & 1286 & 19 & -40\end{array}$ $\begin{array}{llllllllllllllllll}1288 & 6 & -14 & 1288 & 0 & 1 & 1287 & 6 & -8 & 1287 & 0 & 0 & 1287 & 11 & -22 & 1286 & 19 & -40\end{array}$ $\begin{array}{llllllllllllllllll}1288 & 0 & 0 & 1288 & 12 & -12 & 1286 & 10 & -20 & 1287 & 23 & -17 & 1287 & 10 & -20 & 1286 & 0 & 0\end{array}$ $\begin{array}{llllllllllllllllll}1279 & 87 & 18 & 1280 & 83 & -43 & 1279 & 28 & -14 & 1279 & 93 & -72 & 1279 & 49 & -29 & 1279 & 0 & 0\end{array}$ $\begin{array}{llllllllllllllllll}1279 & 87 & 18 & 1279 & 0 & 4 & 1279 & 52 & 29 & 1279 & 0 & 0 & 1279 & 4 & -3 & 1279 & 3 & 14\end{array}$ $\begin{array}{llllllllllllllllll}1279 & 0 & 0 & 1279 & 18 & 14 & 1279 & 16 & -18 & 1279 & 1 & 22 & 1279 & 2 & 9 & 1279 & 3 & 14\end{array}$ $\begin{array}{llllllllllllllllll}1279 & 1 & 49 & 1279 & 61 & 58 & 1279 & 10 & 12 & 1279 & 7 & -2 & 1279 & 4 & 6 & 1279 & 1 & -7\end{array}$ $\begin{array}{rrrrrrrrrrrrrrrrrr}1268 & 0 & 0 & 1269 & 85 & -65 & 1268 & 82 & -65 & 1266 & 0 & 0 & 1266 & 17 & -21 & 1264 & 0 & 0\end{array}$ $\begin{array}{llllllllllllllllll}1268 & 188 & -132 & 1268 & 101 & -74 & 1266 & 25 & 0 & 1265 & 156 & -46 & 1266 & 84 & -19 & 1264 & 37 & -17\end{array}$ $\begin{array}{llllllllllllllllll}1268 & 188 & -132 & 1266 & 100 & -41 & 1266 & 95 & -46 & 1265 & 37 & -45 & 1263 & 22 & -13 & 1264 & 37 & -17\end{array}$ $\begin{array}{rlllllllllllllllll}1267 & 0 & 0 & 1266 & 19 & -9 & 1264 & 18 & -9 & 1265 & 1 & 11 & 1263 & 17 & -6 & 1264 & 0 & 0\end{array}$ $\begin{array}{llllllllllllllllll}1220 & 0 & 0 & 1221 & 9 & 32 & 1220 & 19 & -6 & 1219 & 0 & 0 & 1219 & 5 & -15 & 1215 & 0 & 0\end{array}$ $\begin{array}{llllllllllllllllll}1220 & 21 & 76 & 1221 & 49 & -50 & 1218 & 6 & 18 & 1218 & 7 & -28 & 1218 & 51 & 5 & 1214 & 11 & 27\end{array}$ $\begin{array}{llllllllllllllllll}1220 & 21 & 76 & 1219 & 32 & 44 & 1218 & 65 & -37 & 1218 & 72 & 91 & 1215 & 5 & 10 & 1214 & 11 & 27\end{array}$ $\begin{array}{llllllllllllllllll}1219 & 98 & -213 & 1218 & 34 & -65 & 1216 & 31 & 4 & 1218 & 42 & -82 & 1214 & 13 & 8 & 1214 & 14 & -54\end{array}$ $\begin{array}{llllllllllllllllll}1216 & 0 & 0 & 1216 & 35 & 3 & 1214 & 9 & 9 & 1212 & 0 & 0 & 1213 & 3 & 12 & 1212 & 0 & 0\end{array}$ $\begin{array}{llllllllllllllllll}1216 & 60 & 7 & 1216 & 26 & 3 & 1212 & 8 & 17 & 1212 & 15 & 31 & 1213 & 19 & -32 & 1212 & 1 & 4\end{array}$ $\begin{array}{llllllllllllllllll}1216 & 60 & 7 & 1213 & 8 & 16 & 1212 & 12 & -34 & 1211 & 9 & 27 & 1212 & 3 & 7 & 1212 & 1 & 4\end{array}$ $\begin{array}{llllllllllllllllll}1215 & 0 & 0 & 1212 & 13 & -15 & 1212 & 3 & 10 & 1211 & 17 & -54 & 1212 & 1 & 1 & 1212 & 0 & 0\end{array}$ $\begin{array}{llllllllllllllllll}1198 & 0 & 0 & 1198 & 4 & -22 & 1197 & 2 & -24 & 1197 & 0 & 0 & 1197 & 0 & -2 & 1192 & 0 & 0\end{array}$ $\begin{array}{llllllllllllllllll}1197 & 14 & -77 & 1197 & 6 & -52 & 1197 & 4 & -33 & 1197 & 0 & 1 & 1196 & 113 & -17 & 1191 & 1 & 11\end{array}$ $\begin{array}{llllllllllllllllll}1197 & 14 & -77 & 1197 & 6 & -22 & 1196 & 83 & 93 & 1196 & 175 & 157 & 1191 & 1 & 4 & 1191 & 1 & 11\end{array}$ $\begin{array}{llllllllllllllllll}1197 & 0 & 0 & 1196 & 41 & 59 & 1195 & 130 & -68 & 1196 & 47 & -203 & 1191 & 3 & 9 & 1190 & 33 & 82\end{array}$ $\begin{array}{rlllllllllllllllll}1196 & 0 & 0 & 1196 & 174 & 190 & 1191 & 1 & 7 & 1190 & 0 & 0 & 1190 & 1 & 4 & 1190 & 0 & 0\end{array}$ $\begin{array}{llllllllllllllllll}1196 & 164 & 219 & 1195 & 107 & -205 & 1191 & 0 & 0 & 1190 & 1 & -18 & 1190 & 25 & 32 & 1189 & 28 & -88\end{array}$ $\begin{array}{llllllllllllllllll}1196 & 164 & 219 & 1191 & 1 & -8 & 1190 & 28 & 5 & 1189 & 22 & 41 & 1189 & 23 & -69 & 1189 & 28 & -88\end{array}$ $\begin{array}{llllllllllllllllll}1195 & 72 & -346 & 1189 & 22 & -6 & 1189 & 17 & -40 & 1189 & 25 & -54 & 1189 & 11 & -31 & 1189 & 0 & 0\end{array}$ $\begin{array}{llllllllllllllllll}1146 & 0 & 0 & 1147 & 6 & -22 & 1146 & 30 & 6 & 1143 & 0 & 0 & 1144 & 12 & -46 & 1140 & 0 & 0\end{array}$ $\begin{array}{llllllllllllllllll}1146 & 13 & -50 & 1147 & 56 & 44 & 1143 & 29 & -44 & 1143 & 12 & -38 & 1144 & 89 & 83 & 1140 & 30 & -98\end{array}$ $\begin{array}{llllllllllllllllll}1146 & 13 & -50 & 1144 & 6 & -19 & 1143 & 79 & 93 & 1143 & 26 & -100 & 1141 & 15 & -51 & 1140 & 30 & -98\end{array}$ $\begin{array}{llllllllllllllllll}1146 & 112 & 168 & 1144 & 113 & 90 & 1140 & 102 & 72 & 1143 & 189 & 253 & 1140 & 177 & 178 & 1139 & 305 & 408\end{array}$ $\begin{array}{rlllllllllllllllll}1128 & 0 & 0 & 1129 & 0 & 0 & 1127 & 0 & 0 & 1126 & 0 & 0 & 1126 & 8 & 16 & 1124 & 0 & 0\end{array}$ $\begin{array}{llllllllllllllllll}1128 & 0 & 0 & 1129 & 0 & 0 & 1125 & 5 & -3 & 1126 & 0 & 0 & 1126 & 7 & -10 & 1124 & 13 & -23\end{array}$ $\begin{array}{llllllllllllllllll}1128 & 0 & 0 & 1127 & 0 & 0 & 1125 & 12 & 9 & 1125 & 18 & 30 & 1125 & 7 & -13 & 1124 & 13 & -23\end{array}$ $\begin{array}{lllllllllllllllllr}1128 & 0 & 0 & 1126 & 16 & 4 & 1124 & 8 & -12 & 1125 & 15 & -22 & 1125 & 8 & -12 & 1124 & 0 & 0\end{array}$ $\begin{array}{llllllllllllllllll}1076 & 0 & 0 & 1075 & 4 & -2 & 1077 & 6 & -1 & 1076 & 3 & -3 & 1076 & 5 & 0 & 1077 & 0 & 0\end{array}$ $\begin{array}{llllllllllllllllll}1076 & 0 & 0 & 1075 & 7 & 2 & 1077 & 6 & -1 & 1076 & 0 & 0 & 1076 & 6 & 0 & 1077 & 0 & 0\end{array}$ 
$\begin{array}{llllllllllllllllll}1076 & 12 & -3 & 1074 & 4 & -1 & 1076 & 6 & 0 & 1076 & 2 & -17 & 1076 & 3 & -1 & 1077 & 11 & -2\end{array}$

$\begin{array}{llllllllllllllllll}1076 & 12 & -3 & 1074 & 8 & 1 & 1076 & 6 & -1 & 1076 & 28 & 13 & 1076 & 13 & 0 & 1077 & 11 & -2\end{array}$

$\begin{array}{llllllllllllllllll}1074 & 0 & 0 & 1074 & 23 & 12 & 1076 & 18 & -16 & 1074 & 0 & 0 & 1074 & 19 & 2 & 1076 & 0 & 0\end{array}$

$\begin{array}{llllllllllllllllll}1074 & 44 & 28 & 1074 & 22 & -39 & 1075 & 21 & -16 & 1074 & 39 & 0 & 1074 & 19 & -30 & 1076 & 35 & 2\end{array}$

$\begin{array}{llllllllllllllllll}1074 & 44 & 28 & 1073 & 22 & 18 & 1075 & 20 & -15 & 1074 & 36 & 31 & 1074 & 19 & 4 & 1076 & 35 & 2\end{array}$

$\begin{array}{llllllllllllllllll}1074 & 7 & -130 & 1073 & 28 & -62 & 1074 & 25 & -24 & 1074 & 4 & -96 & 1074 & 22 & -43 & 1076 & 3 & -75\end{array}$

$\begin{array}{llllllllllllllllll}1041 & 0 & 0 & 1040 & 1 & 24 & 1042 & 6 & -9 & 1040 & 0 & 0 & 1041 & 2 & -16 & 1042 & 0 & 0\end{array}$

$\begin{array}{llllllllllllllllll}1041 & 0 & 0 & 1040 & 14 & -35 & 1042 & 7 & 22 & 1040 & 1 & 42 & 1041 & 0 & -5 & 1042 & 1 & -35\end{array}$

$\begin{array}{llllllllllllllllll}1041 & 3 & 72 & 1040 & 1 & 27 & 1042 & 19 & 11 & 1040 & 16 & -28 & 1041 & 10 & -16 & 1042 & 1 & -35\end{array}$

$\begin{array}{llllllllllllllllll}1041 & 3 & 72 & 1039 & 6 & -8 & 1041 & 8 & -16 & 1040 & 11 & -35 & 1041 & 10 & 12 & 1042 & 0 & 0\end{array}$

$\begin{array}{llllllllllllllllll}1040 & 3 & 7 & 1039 & 0 & 3 & 1041 & 2 & 41 & 1040 & 0 & 0 & 1041 & 11 & 90 & 1042 & 0 & 0\end{array}$

$\begin{array}{llllllllllllllllll}1040 & 3 & 7 & 1039 & 6 & 63 & 1041 & 6 & 44 & 1040 & 12 & 138 & 1040 & 28 & -30 & 1042 & 23 & 138\end{array}$

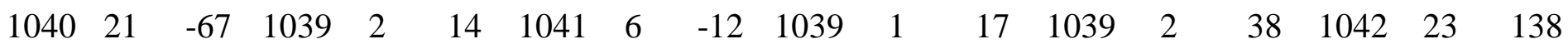

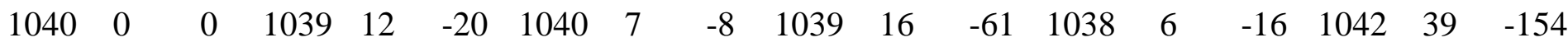

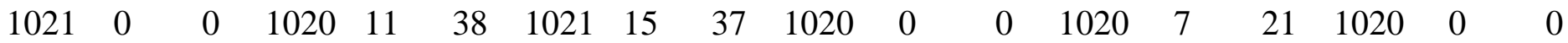

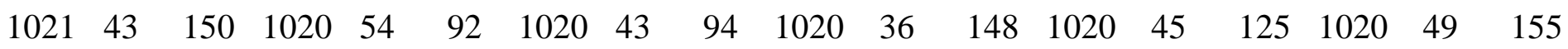

$\begin{array}{llllllllllllllllll}1021 & 43 & 150 & 1020 & 33 & 121 & 1020 & 41 & 107 & 1020 & 61 & 171 & 1020 & 49 & 148 & 1020 & 49 & 155\end{array}$

$\begin{array}{llllllllllllllllll}1020 & 78 & -202 & 1020 & 73 & -143 & 1020 & 67 & -137 & 1020 & 75 & -210 & 1020 & 69 & -184 & 1020 & 68 & -206\end{array}$

$\begin{array}{llllllllllllllllll}985 & 0 & 0 & 990 & 11 & -4 & 992 & 10 & 0 & 990 & 0 & 0 & 991 & 1 & -2 & 992 & 0 & 0\end{array}$

$\begin{array}{llllllllllllllllll}985 & 0 & -1 & 990 & 0 & 1 & 992 & 3 & -4 & 990 & 16 & -3 & 991 & 14 & -4 & 992 & 22 & -8\end{array}$

$\begin{array}{llllllllllllllllll}985 & 0 & -1 & 985 & 1 & 2 & 991 & 5 & -2 & 990 & 0 & 0 & 991 & 12 & -2 & 992 & 22 & -8\end{array}$

$\begin{array}{llllllllllllllllll}985 & 0 & 0 & 985 & 0 & -1 & 991 & 6 & -1 & 990 & 0 & 1 & 991 & 5 & -3 & 992 & 0 & 0\end{array}$

$\begin{array}{llllllllllllllllll}985 & 0 & 1 & 985 & 1 & 2 & 986 & 0 & 1 & 985 & 0 & 0 & 991 & 0 & -1 & 992 & 0 & 0\end{array}$

$\begin{array}{llllllllllllllllll}985 & 1 & 3 & 985 & 0 & 0 & 986 & 0 & 1 & 985 & 1 & -2 & 991 & 2 & 2 & 992 & 0 & 0\end{array}$

$\begin{array}{llllllllllllllllll}985 & 1 & 3 & 985 & 0 & -1 & 985 & 0 & 1 & 985 & 0 & 0 & 986 & 0 & -1 & 992 & 0 & 2\end{array}$

$\begin{array}{rlllllllllllllllll}985 & 0 & 0 & 985 & 1 & 1 & 985 & 1 & 1 & 985 & 3 & 5 & 986 & 1 & 2 & 992 & 0 & 2\end{array}$

$\begin{array}{rlllllllllllllllll}969 & 0 & 0 & 974 & 424 & -39 & 977 & 284 & -41 & 973 & 0 & 0 & 979 & 109 & -19 & 979 & 0 & 0\end{array}$

$\begin{array}{lllllllllllllllllll}961 & 1338 & 69 & 961 & 624 & -13 & 970 & 866 & -82 & 969 & 1306 & -106 & 972 & 1295 & -116 & 971 & 1294 & -186\end{array}$

$\begin{array}{llllllllllllllllll}961 & 1338 & 69 & 956 & 1332 & 49 & 962 & 1010 & 0 & 956 & 1345 & 49 & 970 & 593 & -71 & 971 & 1294 & -186\end{array}$

$\begin{array}{llllllllllllllllll}957 & 0 & 0 & 954 & 276 & 38 & 957 & 472 & 68 & 956 & 0 & 0 & 958 & 618 & 60 & 968 & 0 & 0\end{array}$

$\begin{array}{llllllllllllllllll}938 & 0 & 0 & 942 & 2 & 0 & 944 & 3 & 2 & 942 & 1 & -5 & 944 & 2 & 2 & 944 & 0 & 0\end{array}$

$\begin{array}{llllllllllllllllll}938 & 0 & 0 & 942 & 2 & 0 & 943 & 1 & -3 & 942 & 4 & 6 & 944 & 3 & 3 & 944 & 0 & 0\end{array}$

$\begin{array}{llllllllllllllllll}938 & 0 & 0 & 938 & 2 & -3 & 943 & 1 & -2 & 942 & 0 & 0 & 943 & 2 & -9 & 944 & 5 & 4\end{array}$

$\begin{array}{rlllllllllllllllll}938 & 0 & 0 & 938 & 0 & 0 & 943 & 2 & 0 & 942 & 4 & -2 & 943 & 0 & 0 & 944 & 5 & 4\end{array}$

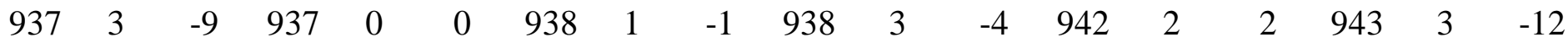

$\begin{array}{llllllllllllllllll}937 & 1 & 3 & 937 & 0 & 1 & 938 & 1 & -1 & 938 & 0 & 0 & 942 & 2 & -2 & 943 & 1 & -2\end{array}$

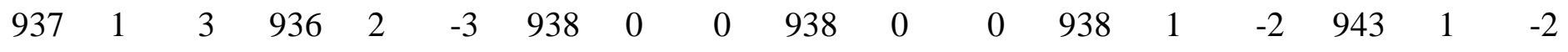

$\begin{array}{llllllllllllllllll}937 & 0 & 0 & 936 & 1 & 2 & 936 & 1 & 0 & 938 & 0 & 0 & 938 & 0 & 0 & 943 & 0 & 0\end{array}$

$\begin{array}{llllllllllllllllll}925 & 0 & 0 & 927 & 27 & 14 & 927 & 12 & -13 & 926 & 16 & 17 & 927 & 14 & 2 & 927 & 0 & 0\end{array}$

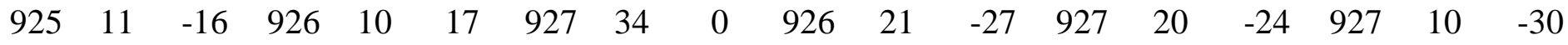

$\begin{array}{llllllllllllllllll}925 & 11 & -16 & 926 & 4 & 1 & 926 & 2 & 6 & 925 & 10 & 1 & 927 & 18 & 21 & 927 & 10 & -30\end{array}$

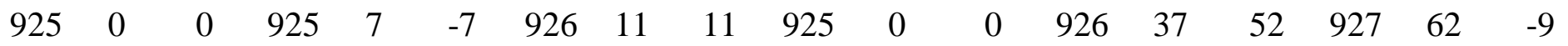

$\begin{array}{llllllllllllllllll}924 & 93 & 74 & 924 & 6 & -10 & 925 & 1 & 11 & 924 & 47 & 1 & 926 & 0 & -3 & 926 & 0 & 0\end{array}$

$\begin{array}{llllllllllllllllll}924 & 4 & -23 & 923 & 4 & -21 & 925 & 18 & 33 & 924 & 6 & 40 & 925 & 30 & 8 & 926 & 15 & 51\end{array}$

$\begin{array}{llllllllllllllllll}924 & 4 & -23 & 923 & 62 & 64 & 924 & 21 & -5 & 923 & 9 & -38 & 925 & 6 & 35 & 926 & 15 & 51\end{array}$

$\begin{array}{llllllllllllllllll}923 & 0 & 0 & 923 & 21 & -4 & 924 & 32 & 50 & 923 & 0 & 0 & 924 & 7 & 1 & 925 & 0 & 0\end{array}$

$\begin{array}{llllllllllllllllll}914 & 3 & -3 & 923 & 1 & -7 & 923 & 33 & 23 & 922 & 31 & 70 & 924 & 16 & 39 & 925 & 0 & 0\end{array}$ 
$\begin{array}{llllllllllllllllll}912 & 5 & -44 & 909 & 6 & -21 & 923 & 7 & 8 & 922 & 17 & 31 & 923 & 39 & 48 & 924 & 63 & 66\end{array}$ $\begin{array}{llllllllllllllllll}912 & 5 & -44 & 908 & 2 & -30 & 912 & 9 & -44 & 910 & 1 & -2 & 923 & 3 & 1 & 924 & 63 & 66\end{array}$

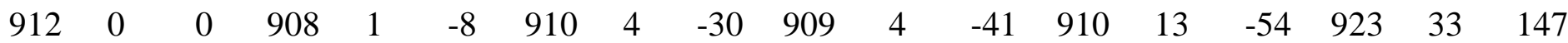
$\begin{array}{llllllllllllllllll}831 & 0 & 0 & 832 & 32 & 6 & 833 & 25 & 10 & 832 & 0 & 0 & 833 & 28 & 0 & 833 & 0 & 0\end{array}$ $\begin{array}{llllllllllllllllll}831 & 58 & 28 & 831 & 13 & 17 & 832 & 33 & -15 & 832 & 71 & 28 & 832 & 37 & 21 & 833 & 62 & 10\end{array}$ $\begin{array}{llllllllllllllllll}831 & 58 & 28 & 831 & 6 & -13 & 832 & 3 & 4 & 832 & 44 & 17 & 832 & 10 & -7 & 833 & 62 & 10\end{array}$ $\begin{array}{llllllllllllllllll}830 & 0 & 0 & 831 & 47 & -16 & 832 & 18 & 28 & 831 & 1 & -5 & 832 & 33 & -15 & 833 & 0 & 0\end{array}$ $\begin{array}{llllllllllllllllll}830 & 0 & 0 & 830 & 23 & 30 & 831 & 25 & -20 & 831 & 0 & 0 & 832 & 10 & 19 & 833 & 0 & 0\end{array}$ $\begin{array}{llllllllllllllllll}830 & 8 & -36 & 830 & 7 & 15 & 831 & 26 & -7 & 831 & 22 & -38 & 831 & 3 & 11 & 832 & 9 & -25\end{array}$ $\begin{array}{llllllllllllllllll}830 & 8 & -36 & 829 & 10 & -34 & 830 & 2 & 12 & 831 & 6 & -33 & 831 & 21 & -26 & 832 & 9 & -25\end{array}$ $\begin{array}{llllllllllllllllll}829 & 2 & 16 & 829 & 6 & -9 & 830 & 11 & -15 & 831 & 4 & 26 & 831 & 6 & -3 & 832 & 4 & 29\end{array}$ $\begin{array}{llllllllllllllllll}808 & 0 & 0 & 808 & 3 & 0 & 808 & 1 & 3 & 808 & 7 & -10 & 808 & 2 & 0 & 809 & 0 & 0\end{array}$

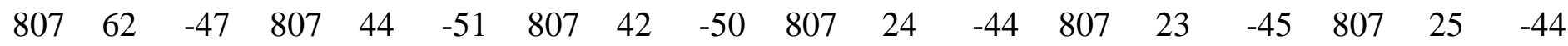
$\begin{array}{llllllllllllllllll}807 & 62 & -47 & 807 & 65 & -48 & 807 & 43 & -48 & 807 & 62 & -44 & 807 & 42 & -48 & 807 & 25 & -44\end{array}$ $\begin{array}{llllllllllllllllll}805 & 0 & 0 & 805 & 0 & 0 & 806 & 1 & -1 & 805 & 0 & 0 & 805 & 0 & 0 & 806 & 0 & 0\end{array}$ $\begin{array}{llllllllllllllllll}776 & 2 & 10 & 777 & 62 & -11 & 778 & 75 & -53 & 777 & 1 & -10 & 778 & 23 & -41 & 778 & 26 & -47\end{array}$ $\begin{array}{lllllllllllllllllll}774 & 316 & -183 & 776 & 9 & -34 & 777 & 156 & 94 & 776 & 445 & -111 & 777 & 278 & -97 & 777 & 320 & -139\end{array}$ $\begin{array}{llllllllllllllllll}774 & 316 & -183 & 775 & 308 & -105 & 776 & 24 & -21 & 776 & 0 & 0 & 776 & 45 & -99 & 777 & 320 & -139\end{array}$ $\begin{array}{llllllllllllllllll}774 & 0 & 0 & 775 & 317 & -89 & 776 & 127 & -107 & 776 & 4 & -33 & 776 & 55 & -51 & 777 & 0 & 0\end{array}$ $\begin{array}{rlllllllllllllllll}774 & 0 & 0 & 775 & 24 & 29 & 775 & 425 & 18 & 775 & 0 & 0 & 776 & 112 & 86 & 777 & 0 & 0\end{array}$ $\begin{array}{llllllllllllllllll}773 & 271 & 196 & 774 & 5 & 29 & 775 & 43 & -45 & 774 & 4 & -5 & 776 & 185 & 99 & 777 & 133 & 178\end{array}$

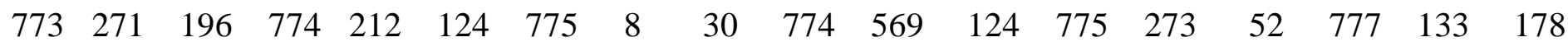

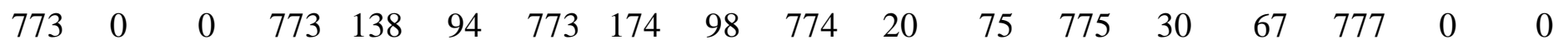
$\begin{array}{llllllllllllllllll}771 & 6 & -122 & 772 & 2 & 52 & 773 & 22 & 200 & 772 & 11 & 157 & 773 & 44 & 292 & 774 & 79 & 388\end{array}$ $\begin{array}{llllllllllllllllll}768 & 378 & 91 & 768 & 371 & -35 & 769 & 330 & -5 & 769 & 301 & -100 & 769 & 279 & -95 & 770 & 288 & -83\end{array}$ $\begin{array}{rllllllllllllllllll}768 & 378 & 91 & 768 & 374 & 97 & 769 & 360 & -32 & 768 & 325 & 113 & 768 & 315 & 6 & 770 & 288 & -83\end{array}$ $\begin{array}{llllllllllllllllll}761 & 0 & 0 & 761 & 0 & 0 & 761 & 0 & 0 & 761 & 0 & 0 & 761 & 0 & 0 & 762 & 0 & 0\end{array}$ $\begin{array}{llllllllllllllllll}739 & 0 & 0 & 740 & 26 & -13 & 742 & 21 & -18 & 741 & 59 & -25 & 742 & 20 & -14 & 744 & 0 & 0\end{array}$ $\begin{array}{llllllllllllllllll}737 & 8 & 6 & 737 & 8 & 7 & 738 & 12 & 13 & 738 & 10 & 9 & 739 & 49 & 17 & 740 & 58 & 26\end{array}$ $\begin{array}{llllllllllllllllll}737 & 8 & 6 & 736 & 24 & 18 & 738 & 27 & 16 & 738 & 86 & 7 & 739 & 23 & 21 & 740 & 58 & 26\end{array}$ $\begin{array}{llllllllllllllllll}736 & 0 & 0 & 735 & 2 & 1 & 736 & 8 & 5 & 737 & 0 & 0 & 737 & 18 & -2 & 738 & 0 & 0\end{array}$ $\begin{array}{llllllllllllllllll}734 & 0 & 0 & 734 & 11 & -34 & 736 & 8 & -12 & 735 & 24 & -58 & 736 & 7 & -15 & 737 & 0 & 0\end{array}$ $\begin{array}{llllllllllllllllll}734 & 0 & -4 & 734 & 10 & 16 & 736 & 6 & -10 & 734 & 22 & -35 & 735 & 10 & -9 & 736 & 1 & -10\end{array}$

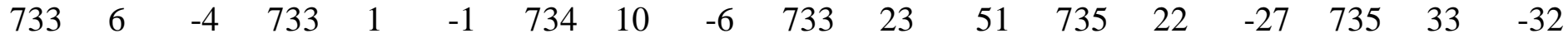

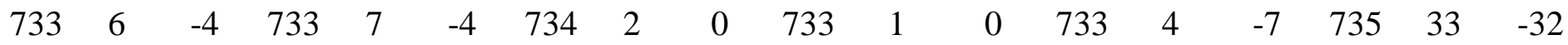
$\begin{array}{rlllllllllllllllll}733 & 0 & 0 & 732 & 3 & -2 & 733 & 5 & -2 & 733 & 0 & 0 & 733 & 2 & 1 & 735 & 0 & 0\end{array}$ $\begin{array}{lllllllllllllllllll}725 & 228 & 35 & 726 & 217 & -6 & 728 & 185 & -63 & 727 & 224 & -27 & 728 & 219 & -90 & 730 & 153 & -158\end{array}$ $\begin{array}{lllllllllllllllllll}725 & 228 & 35 & 725 & 212 & -32 & 727 & 225 & -73 & 726 & 141 & -90 & 728 & 143 & -125 & 730 & 153 & -158\end{array}$ $\begin{array}{llllllllllllllllll}716 & 0 & 0 & 716 & 10 & 8 & 718 & 17 & 9 & 718 & 0 & 0 & 719 & 10 & -1 & 722 & 0 & 0\end{array}$

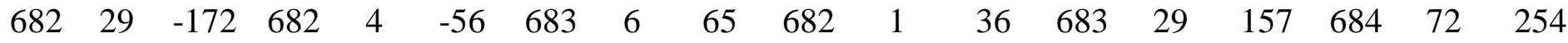
$\begin{array}{lllllllllllllllllll}678 & 173 & 56 & 678 & 180 & 102 & 680 & 152 & 46 & 679 & 193 & 151 & 680 & 177 & 87 & 681 & 138 & 41\end{array}$ $\begin{array}{lllllllllllllllllll}678 & 173 & 56 & 677 & 155 & 8 & 679 & 155 & 48 & 677 & 120 & -24 & 679 & 110 & 16 & 681 & 138 & 41\end{array}$ $\begin{array}{llllllllllllllllll}674 & 0 & 0 & 674 & 9 & -2 & 675 & 20 & -10 & 675 & 0 & 0 & 676 & 15 & -9 & 678 & 0 & 0\end{array}$ $\begin{array}{rlllllllllllllllll}617 & 0 & 0 & 616 & 122 & -34 & 616 & 238 & -55 & 615 & 12 & 82 & 615 & 114 & 7 & 616 & 0 & 0\end{array}$ $\begin{array}{llllllllllllllllll}615 & 259 & -159 & 614 & 6 & 39 & 615 & 0 & -3 & 614 & 109 & 50 & 614 & 131 & 54 & 614 & 153 & 58\end{array}$ $\begin{array}{llllllllllllllllll}615 & 259 & -159 & 614 & 233 & -153 & 614 & 8 & 51 & 613 & 300 & -194 & 613 & 12 & 20 & 614 & 153 & 58\end{array}$

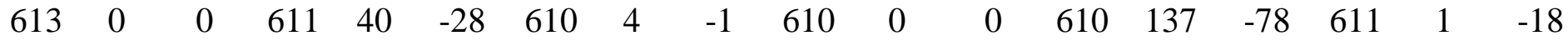


$\begin{array}{lllllllllllllllllll}607 & 139 & 235 & 608 & 195 & 23 & 609 & 277 & -100 & 609 & 30 & -1 & 609 & 18 & -5 & 608 & 0 & 0\end{array}$

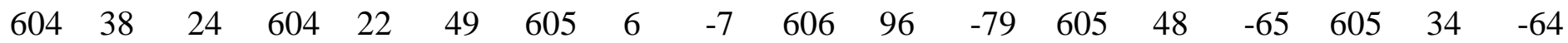
$\begin{array}{llllllllllllllllll}604 & 38 & 24 & 604 & 21 & 26 & 605 & 19 & 37 & 604 & 10 & 26 & 604 & 3 & -1 & 605 & 34 & -64\end{array}$ $\begin{array}{llllllllllllllllll}602 & 0 & 0 & 602 & 1 & -1 & 603 & 2 & 4 & 603 & 0 & 0 & 603 & 1 & 1 & 604 & 0 & 0\end{array}$ $\begin{array}{llllllllllllllllll}569 & 0 & 0 & 569 & 22 & 13 & 569 & 17 & 13 & 569 & 10 & 10 & 569 & 5 & 5 & 568 & 0 & 0\end{array}$ $\begin{array}{llllllllllllllllll}569 & 90 & 34 & 569 & 47 & 22 & 569 & 41 & 3 & 569 & 0 & 0 & 569 & 29 & -1 & 568 & 3 & -3\end{array}$ $\begin{array}{llllllllllllllllll}569 & 90 & 34 & 569 & 0 & 0 & 569 & 35 & 1 & 569 & 0 & -3 & 568 & 21 & -7 & 568 & 3 & -3\end{array}$ $\begin{array}{rlllllllllllllllll}569 & 0 & 0 & 569 & 73 & -4 & 568 & 7 & -5 & 569 & 106 & -11 & 568 & 1 & -2 & 568 & 0 & 0\end{array}$ $\begin{array}{lllllllllllllllllll}545 & 154 & 276 & 545 & 246 & 104 & 545 & 326 & -27 & 545 & 43 & 23 & 544 & 144 & -57 & 543 & 1 & -20\end{array}$ $\begin{array}{llllllllllllllllll}543 & 233 & -118 & 543 & 201 & -103 & 542 & 2 & -6 & 542 & 186 & -87 & 541 & 115 & 14 & 541 & 135 & 12\end{array}$

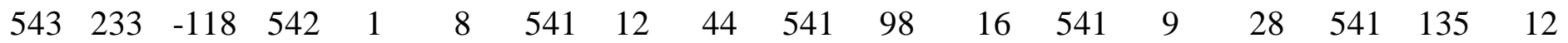
$\begin{array}{llllllllllllllllll}539 & 0 & 0 & 539 & 1 & -1 & 539 & 1 & 1 & 539 & 0 & 0 & 539 & 3 & 0 & 538 & 0 & 0\end{array}$ $\begin{array}{rllllllllllllllllll}525 & 0 & 0 & 524 & 202 & -3 & 524 & 475 & -97 & 524 & 60 & 176 & 524 & 316 & -12 & 525 & 0 & 0\end{array}$ $\begin{array}{llllllllllllllllll}522 & 164 & -54 & 522 & 151 & -50 & 522 & 81 & -3 & 524 & 111 & -32 & 523 & 51 & 52 & 522 & 713 & -114\end{array}$

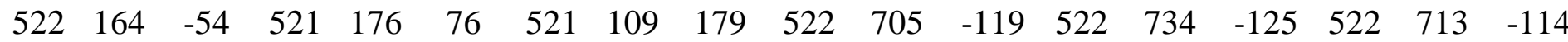
$\begin{array}{llllllllllllllllll}519 & 63 & 123 & 520 & 159 & 13 & 520 & 317 & -73 & 520 & 7 & 35 & 520 & 112 & 79 & 520 & 31 & 165\end{array}$ $\begin{array}{llllllllllllllllll}509 & 0 & 0 & 510 & 2 & 1 & 510 & 1 & 1 & 513 & 0 & 0 & 512 & 0 & 0 & 510 & 0 & 0\end{array}$

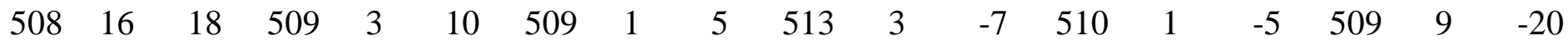
$\begin{array}{llllllllllllllllll}508 & 16 & 18 & 508 & 11 & 14 & 509 & 5 & 11 & 508 & 13 & 17 & 508 & 1 & 2 & 509 & 9 & -20\end{array}$ $\begin{array}{llllllllllllllllll}507 & 0 & 0 & 507 & 0 & 1 & 506 & 1 & -2 & 507 & 0 & 0 & 506 & 0 & 0 & 506 & 2 & -1\end{array}$ $\begin{array}{llllllllllllllllll}505 & 1 & -1 & 504 & 3 & 1 & 506 & 0 & -1 & 503 & 3 & 16 & 505 & 7 & -2 & 506 & 0 & 0\end{array}$ $\begin{array}{llllllllllllllllll}504 & 3 & -1 & 503 & 0 & -1 & 504 & 2 & 1 & 502 & 24 & 9 & 504 & 0 & 0 & 504 & 6 & 2\end{array}$ $\begin{array}{llllllllllllllllll}504 & 3 & -1 & 502 & 2 & 1 & 503 & 1 & 0 & 498 & 5 & 9 & 501 & 4 & 6 & 504 & 6 & 2\end{array}$ $\begin{array}{llllllllllllllllll}502 & 0 & 0 & 500 & 1 & 1 & 502 & 2 & 3 & 498 & 0 & 0 & 501 & 1 & 1 & 503 & 0 & 0\end{array}$ $\begin{array}{lllllllllllllllllll}496 & 0 & 0 & 494 & 44 & 5 & 495 & 107 & -19 & 494 & 11 & 42 & 494 & 91 & -5 & 495 & 0 & 0\end{array}$

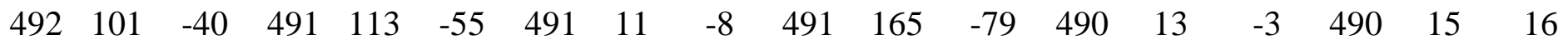

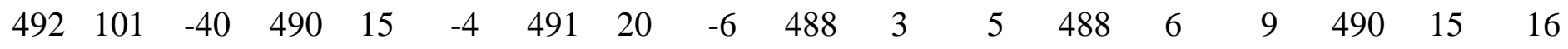
$\begin{array}{llllllllllllllllll}490 & 0 & 0 & 488 & 2 & -3 & 487 & 0 & 0 & 486 & 0 & 0 & 485 & 0 & 0 & 486 & 0 & 0\end{array}$ $\begin{array}{llllllllllllllllll}462 & 0 & 0 & 462 & 1 & -5 & 464 & 0 & -5 & 463 & 0 & 0 & 464 & 0 & 3 & 465 & 0 & 0\end{array}$ $\begin{array}{llllllllllllllllll}458 & 2 & -16 & 457 & 0 & -2 & 459 & 6 & -3 & 458 & 0 & -2 & 459 & 4 & 2 & 459 & 2 & 14\end{array}$

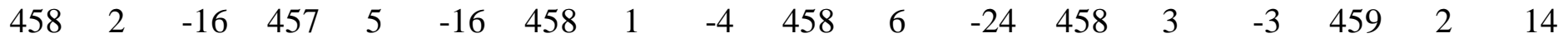
$\begin{array}{llllllllllllllllll}450 & 0 & 0 & 449 & 13 & -1 & 449 & 29 & 10 & 449 & 10 & -19 & 448 & 16 & 1 & 449 & 0 & 0\end{array}$ $\begin{array}{llllllllllllllllll}437 & 0 & 0 & 434 & 3 & -15 & 433 & 1 & -14 & 433 & 0 & 0 & 431 & 0 & 6 & 430 & 0 & 0\end{array}$ $\begin{array}{llllllllllllllllll}427 & 265 & -83 & 425 & 244 & 53 & 424 & 83 & 50 & 426 & 241 & 165 & 423 & 9 & 32 & 421 & 387 & -261\end{array}$

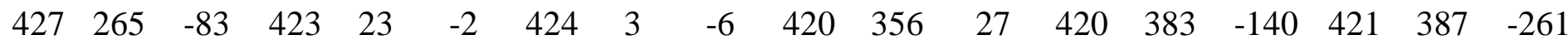
$\begin{array}{llllllllllllllllll}421 & 1 & 20 & 418 & 334 & -48 & 418 & 639 & -71 & 417 & 34 & -19 & 415 & 439 & 65 & 416 & 183 & 357\end{array}$ $\begin{array}{llllllllllllllllll}394 & 0 & 0 & 399 & 407 & 48 & 403 & 319 & 20 & 399 & 187 & 309 & 403 & 292 & 14 & 403 & 0 & 0\end{array}$ $\begin{array}{llllllllllllllllll}388 & 397 & -28 & 397 & 114 & -97 & 399 & 308 & -194 & 398 & 543 & -242 & 402 & 240 & 25 & 402 & 0 & 0\end{array}$

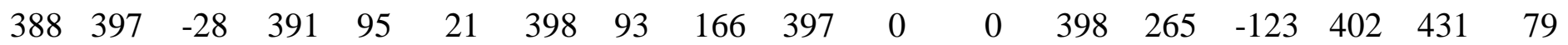
$\begin{array}{llllllllllllllllll}388 & 0 & 0 & 387 & 259 & -33 & 390 & 79 & 18 & 396 & 240 & -198 & 397 & 130 & 106 & 402 & 431 & 79\end{array}$ $\begin{array}{rlllllllllllllllll}384 & 18 & 15 & 384 & 72 & 19 & 389 & 121 & 4 & 383 & 212 & 6 & 390 & 19 & -33 & 391 & 0 & 0\end{array}$

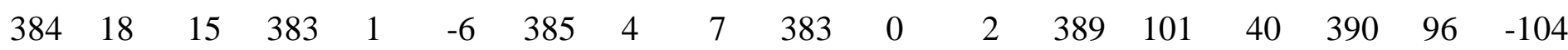

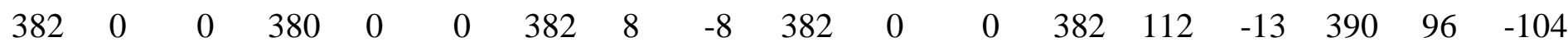
$\begin{array}{llllllllllllllllll}380 & 28 & 64 & 380 & 63 & 32 & 381 & 117 & -1 & 381 & 0 & 0 & 382 & 0 & 0 & 388 & 159 & 139\end{array}$ $\begin{array}{llllllllllllllllll}331 & 0 & 0 & 330 & 69 & 4 & 332 & 32 & 2 & 330 & 5 & 23 & 331 & 1 & 0 & 333 & 0 & 0\end{array}$ $\begin{array}{llllllllllllllllll}330 & 328 & 8 & 328 & 56 & 17 & 331 & 10 & 22 & 329 & 149 & -25 & 329 & 2 & -1 & 332 & 9 & 30\end{array}$ $\begin{array}{llllllllllllllllll}330 & 328 & 8 & 328 & 302 & -21 & 330 & 106 & -3 & 329 & 34 & 94 & 329 & 4 & 8 & 331 & 16 & 0\end{array}$ 
$\begin{array}{llllllllllllllllll}329 & 55 & 145 & 328 & 34 & 84 & 330 & 5 & 2 & 328 & 0 & 0 & 329 & 50 & 55 & 331 & 16 & 0\end{array}$ $\begin{array}{llllllllllllllllll}328 & 15 & -1 & 327 & 1 & 1 & 330 & 27 & -2 & 327 & 2 & -1 & 328 & 5 & 0 & 330 & 1 & -2\end{array}$ $\begin{array}{llllllllllllllllll}328 & 15 & -1 & 327 & 24 & 29 & 329 & 116 & 60 & 327 & 166 & -28 & 327 & 102 & -17 & 330 & 1 & -2\end{array}$ $\begin{array}{llllllllllllllllll}327 & 0 & 0 & 326 & 9 & -4 & 328 & 6 & -3 & 326 & 0 & 0 & 327 & 0 & 0 & 329 & 0 & 0\end{array}$ $\begin{array}{llllllllllllllllll}325 & 0 & 0 & 324 & 7 & 1 & 326 & 4 & 3 & 325 & 0 & 0 & 325 & 0 & 0 & 328 & 0 & 0\end{array}$ $\begin{array}{rlllllllllllllllll}308 & 0 & 0 & 307 & 291 & 37 & 307 & 696 & -36 & 306 & 49 & 131 & 306 & 405 & 7 & 307 & 0 & 0\end{array}$ $\begin{array}{llllllllllllllllll}306 & 0 & 0 & 305 & 117 & 71 & 305 & 32 & -50 & 304 & 510 & -258 & 303 & 418 & -194 & 302 & 523 & -153\end{array}$ $\begin{array}{llllllllllllllllll}305 & 226 & 131 & 304 & 96 & -42 & 303 & 32 & 50 & 303 & 359 & 74 & 302 & 11 & 27 & 302 & 523 & -153\end{array}$ $\begin{array}{llllllllllllllllll}305 & 226 & 131 & 302 & 165 & 23 & 300 & 0 & 0 & 302 & 0 & 0 & 298 & 108 & -15 & 298 & 0 & 0\end{array}$ $\begin{array}{llllllllllllllllll}266 & 509 & -173 & 268 & 179 & 43 & 271 & 670 & 70 & 269 & 68 & 11 & 270 & 549 & 127 & 271 & 261 & 350\end{array}$ $\begin{array}{llllllllllllllllll}266 & 509 & -173 & 265 & 209 & -70 & 268 & 8 & -13 & 268 & 224 & 68 & 268 & 212 & 46 & 269 & 230 & 26\end{array}$ $\begin{array}{llllllllllllllllll}266 & 0 & 0 & 265 & 257 & -63 & 266 & 4 & 2 & 265 & 390 & -84 & 266 & 26 & 6 & 269 & 230 & 26\end{array}$ $\begin{array}{llllllllllllllllll}264 & 5 & 37 & 264 & 290 & -72 & 265 & 274 & -68 & 265 & 0 & 0 & 265 & 11 & -2 & 266 & 0 & 0\end{array}$ $\begin{array}{llllllllllllllllll}218 & 1 & -11 & 220 & 1 & -1 & 223 & 1 & -3 & 220 & 3 & 5 & 221 & 0 & -4 & 223 & 1 & -8\end{array}$ $\begin{array}{llllllllllllllllll}218 & 2 & 3 & 218 & 1 & -8 & 222 & 1 & -1 & 220 & 3 & -16 & 220 & 0 & 2 & 223 & 0 & -1\end{array}$ $\begin{array}{llllllllllllllllll}218 & 2 & 3 & 218 & 4 & 4 & 219 & 1 & -7 & 218 & 17 & 5 & 219 & 2 & -6 & 223 & 0 & -1\end{array}$ $\begin{array}{llllllllllllllllll}217 & 0 & 0 & 217 & 3 & 1 & 219 & 2 & 2 & 218 & 0 & -1 & 217 & 4 & -1 & 222 & 0 & 0\end{array}$ $\begin{array}{llllllllllllllllll}215 & 0 & 0 & 216 & 17 & -3 & 218 & 9 & -1 & 216 & 0 & 0 & 216 & 2 & -1 & 219 & 0 & 0\end{array}$ $\begin{array}{rlllllllllllllllll}215 & 0 & 0 & 214 & 0 & 0 & 217 & 7 & -1 & 215 & 54 & -5 & 216 & 0 & 0 & 219 & 0 & 0\end{array}$ $\begin{array}{llllllllllllllllll}214 & 58 & -6 & 214 & 44 & -6 & 215 & 26 & -5 & 215 & 0 & 0 & 215 & 32 & -6 & 218 & 6 & -3\end{array}$ $\begin{array}{llllllllllllllllll}214 & 58 & -6 & 213 & 38 & -7 & 214 & 23 & -3 & 214 & 10 & -6 & 213 & 6 & -3 & 218 & 6 & -3\end{array}$ $\begin{array}{llllllllllllllllll}202 & 0 & 0 & 202 & 1 & 1 & 203 & 0 & 0 & 202 & 0 & 0 & 203 & 0 & 0 & 204 & 0 & 0\end{array}$ $\begin{array}{llllllllllllllllll}193 & 126 & 1 & 194 & 9 & 9 & 195 & 1 & 2 & 195 & 42 & -10 & 195 & 48 & -18 & 196 & 47 & -25\end{array}$ $\begin{array}{llllllllllllllllll}193 & 126 & 1 & 193 & 134 & 13 & 194 & 18 & 19 & 193 & 163 & 32 & 194 & 8 & 13 & 196 & 47 & -25\end{array}$ $\begin{array}{rlllllllllllllllll}185 & 0 & 0 & 185 & 53 & 0 & 186 & 116 & -9 & 185 & 12 & 16 & 185 & 78 & 1 & 187 & 0 & 0\end{array}$ $\begin{array}{llllllllllllllllll}158 & 3 & -4 & 151 & 4 & -1 & 165 & 3 & 1 & 152 & 4 & 3 & 151 & 5 & 5 & 163 & 10 & -10\end{array}$ $\begin{array}{llllllllllllllllll}158 & 3 & -4 & 150 & 7 & 4 & 163 & 9 & -1 & 151 & 9 & 3 & 151 & 1 & 2 & 163 & 10 & -10\end{array}$ $\begin{array}{rlllllllllllllllll}157 & 0 & 0 & 146 & 1 & 0 & 163 & 4 & -4 & 147 & 0 & 0 & 146 & 4 & -8 & 163 & 0 & 0\end{array}$ $\begin{array}{llllllllllllllllll}157 & 0 & 0 & 145 & 8 & 7 & 162 & 16 & -4 & 146 & 9 & 1 & 146 & 4 & -5 & 163 & 0 & 0\end{array}$ $\begin{array}{llllllllllllllllll}149 & 1 & 1 & 142 & 2 & 3 & 154 & 8 & 1 & 143 & 16 & 25 & 143 & 1 & 0 & 155 & 11 & -4\end{array}$ $\begin{array}{llllllllllllllllll}149 & 1 & 1 & 141 & 1 & 3 & 152 & 7 & -1 & 143 & 17 & -7 & 141 & 39 & -17 & 154 & 20 & 6\end{array}$ $\begin{array}{llllllllllllllllll}149 & 4 & -7 & 141 & 20 & -16 & 149 & 6 & 9 & 142 & 0 & 0 & 141 & 12 & -9 & 154 & 20 & 6\end{array}$ $\begin{array}{llllllllllllllllll}147 & 0 & 0 & 139 & 12 & -7 & 147 & 15 & 15 & 139 & 13 & -19 & 141 & 25 & 9 & 153 & 0 & 0\end{array}$ $\begin{array}{llllllllllllllllll}145 & 35 & 34 & 133 & 4 & 10 & 143 & 3 & 6 & 134 & 0 & 0 & 136 & 0 & 0 & 148 & 1 & 9\end{array}$ $\begin{array}{llllllllllllllllll}145 & 35 & 34 & 132 & 3 & 4 & 142 & 5 & 5 & 129 & 4 & 4 & 133 & 3 & 8 & 148 & 1 & 9\end{array}$ $\begin{array}{llllllllllllllllll}141 & 0 & 0 & 131 & 35 & 26 & 137 & 47 & 0 & 128 & 4 & -10 & 131 & 7 & 14 & 144 & 0 & 0\end{array}$ $\begin{array}{rlllllllllllllllll}135 & 0 & 0 & 127 & 26 & -1 & 136 & 10 & -6 & 123 & 14 & 24 & 131 & 8 & 11 & 137 & 0 & 0\end{array}$ $\begin{array}{llllllllllllllllll}134 & 0 & -3 & 126 & 0 & 0 & 131 & 32 & -8 & 123 & 0 & 0 & 130 & 10 & 5 & 136 & 40 & -6\end{array}$ $\begin{array}{rrrrrrrrrrrrrrrrrr}128 & 0 & 0 & 123 & 5 & -5 & 128 & 38 & 6 & 120 & 0 & -1 & 127 & 52 & 0 & 131 & 0 & 0\end{array}$ $\begin{array}{llllllllllllllllll}119 & 212 & -11 & 118 & 238 & 9 & 120 & 53 & 4 & 118 & 4 & -6 & 120 & 3 & -2 & 122 & 3 & -1\end{array}$ $\begin{array}{llllllllllllllllll}119 & 212 & -11 & 118 & 46 & 9 & 119 & 58 & 9 & 116 & 238 & 26 & 119 & 44 & 11 & 122 & 3 & -1\end{array}$ $\begin{array}{rlllllllllllllllll}115 & 0 & 0 & 114 & 2 & -2 & 114 & 0 & 0 & 114 & 0 & 0 & 113 & 0 & 0 & 114 & 0 & 0\end{array}$ $\begin{array}{llllllllllllllllll}107 & 1 & 8 & 107 & 0 & 2 & 108 & 0 & -2 & 107 & 0 & -2 & 107 & 0 & -2 & 108 & 0 & -2\end{array}$ $\begin{array}{llllllllllllllllll}107 & 1 & 8 & 107 & 0 & 3 & 108 & 0 & -2 & 106 & 3 & 5 & 107 & 0 & -1 & 108 & 0 & -2\end{array}$ $\begin{array}{llllllllllllllllll}101 & 0 & 0 & 101 & 0 & 0 & 102 & 0 & 1 & 101 & 0 & 0 & 101 & 0 & 1 & 103 & 0 & 0\end{array}$ $\begin{array}{llllllllllllllllll}88 & 4 & -9 & 89 & 3 & -6 & 89 & 0 & -2 & 89 & 3 & -5 & 90 & 0 & -2 & 90 & 1 & 5\end{array}$ $\begin{array}{llllllllllllllllll}88 & 4 & -9 & 88 & 7 & -13 & 89 & 3 & -7 & 89 & 11 & -19 & 89 & 1 & -5 & 90 & 1 & 5\end{array}$ 
$\begin{array}{lllllllllllllllllll}85 & 0 & 0 & 84 & 16 & -2 & 85 & 50 & -6 & 84 & 0 & 0 & 85 & 29 & 0 & 85 & 0 & 0\end{array}$ $\begin{array}{llllllllllllllllll}80 & 0 & 0 & 81 & 22 & 3 & 82 & 32 & 7 & 82 & 10 & 5 & 82 & 14 & 7 & 84 & 19 & 8\end{array}$ $\begin{array}{lllllllllllllllllll}77 & 0 & 0 & 76 & 19 & -2 & 81 & 3 & -1 & 77 & 0 & 0 & 80 & 10 & -1 & 83 & 0 & 0\end{array}$ $\begin{array}{llllllllllllllllll}76 & 28 & -1 & 74 & 4 & 2 & 78 & 10 & 1 & 75 & 69 & -5 & 78 & 32 & -1 & 80 & 3 & 1\end{array}$ $\begin{array}{llllllllllllllllll}76 & 28 & -1 & 73 & 36 & -1 & 77 & 3 & 0 & 75 & 38 & -3 & 77 & 5 & 1 & 80 & 3 & 1\end{array}$ $\begin{array}{llllllllllllllllll}74 & 0 & 0 & 72 & 1 & 0 & 75 & 27 & -1 & 72 & 6 & 4 & 73 & 3 & 0 & 78 & 0 & 0\end{array}$ $\begin{array}{llllllllllllllllll}73 & 0 & 0 & 71 & 19 & 0 & 73 & 3 & 1 & 71 & 0 & 0 & 71 & 1 & 0 & 74 & 0 & 0\end{array}$ $\begin{array}{llllllllllllllllll}66 & 61 & 6 & 65 & 24 & 3 & 67 & 6 & 2 & 65 & 0 & 0 & 67 & 30 & -2 & 68 & 5 & -3\end{array}$ $\begin{array}{llllllllllllllllll}66 & 61 & 6 & 64 & 25 & 4 & 67 & 6 & 2 & 65 & 29 & 3 & 66 & 4 & 1 & 68 & 5 & -3\end{array}$ $\begin{array}{llllllllllllllllll}62 & 0 & 0 & 61 & 21 & 2 & 63 & 75 & 6 & 64 & 53 & 3 & 66 & 13 & 4 & 65 & 0 & 0\end{array}$ $\begin{array}{llllllllllllllllll}61 & 0 & 0 & 60 & 19 & 1 & 63 & 81 & 3 & 62 & 0 & 0 & 64 & 8 & 0 & 64 & 0 & 0\end{array}$ $\begin{array}{llllllllllllllllll}61 & 90 & -1 & 60 & 54 & -1 & 62 & 8 & 0 & 62 & 0 & 0 & 61 & 43 & 7 & 61 & 127 & 9\end{array}$ $\begin{array}{rlllllllllllllllll}61 & 90 & -1 & 58 & 50 & -1 & 61 & 13 & 0 & 60 & 65 & 2 & 60 & 104 & 5 & 61 & 127 & 9\end{array}$ $\begin{array}{llllllllllllllllll}54 & 0 & 0 & 52 & 1 & -1 & 53 & 8 & -4 & 49 & 0 & -2 & 49 & 3 & -5 & 53 & 8 & -10\end{array}$ $\begin{array}{llllllllllllllllll}48 & 0 & 0 & 48 & 1 & 0 & 48 & 4 & 0 & 48 & 0 & 0 & 48 & 2 & 0 & 48 & 0 & 0\end{array}$ $\begin{array}{llllllllllllllllll}41 & 0 & 0 & 42 & 4 & 0 & 43 & 1 & 0 & 43 & 11 & 3 & 43 & 0 & 0 & 44 & 0 & 0\end{array}$ $\begin{array}{llllllllllllllllll}41 & 3 & 0 & 41 & 0 & 0 & 42 & 3 & -1 & 42 & 0 & 0 & 43 & 2 & 1 & 43 & 47 & -4\end{array}$ $\begin{array}{llllllllllllllllll}41 & 3 & 0 & 40 & 1 & 0 & 41 & 3 & 0 & 40 & 23 & 1 & 41 & 22 & 0 & 43 & 47 & -4\end{array}$ $\begin{array}{llllllllllllllllll}38 & 2 & 3 & 37 & 121 & -3 & 39 & 188 & -4 & 39 & 28 & -4 & 39 & 97 & 3 & 39 & 87 & 9\end{array}$ $\begin{array}{llllllllllllllllll}36 & 0 & 0 & 35 & 3 & 0 & 37 & 37 & -3 & 36 & 0 & 0 & 37 & 29 & -3 & 38 & 0 & 0\end{array}$ $\begin{array}{llllllllllllllllll}35 & 17 & 4 & 35 & 22 & 4 & 36 & 70 & -6 & 35 & 12 & 6 & 35 & 312 & -17 & 37 & 340 & -28\end{array}$ $\begin{array}{llllllllllllllllll}35 & 17 & 4 & 34 & 43 & -4 & 36 & 58 & -3 & 35 & 367 & -25 & 35 & 157 & -14 & 37 & 340 & -28\end{array}$ $\begin{array}{llllllllllllllllll}33 & 0 & 0 & 32 & 53 & 1 & 34 & 68 & 6 & 32 & 18 & 4 & 33 & 71 & 9 & 35 & 0 & 0\end{array}$ $\begin{array}{llllllllllllllllll}30 & 159 & 9 & 30 & 139 & 0 & 31 & 109 & 0 & 30 & 37 & 1 & 29 & 93 & 1 & 31 & 39 & -5\end{array}$ $\begin{array}{llllllllllllllllll}30 & 159 & 9 & 29 & 212 & 7 & 31 & 132 & -1 & 28 & 0 & 1 & 29 & 65 & -5 & 31 & 39 & -5\end{array}$ $\begin{array}{llllllllllllllllll}25 & 0 & 0 & 22 & 17 & 3 & 24 & 28 & -1 & 28 & 24 & 4 & 25 & 50 & -12 & 24 & 59 & 8\end{array}$ $\begin{array}{llllllllllllllllll}24 & 24 & 7 & 22 & 33 & -16 & 23 & 25 & 6 & 26 & 274 & -16 & 22 & 7 & 2 & 23 & 1 & 4\end{array}$ $\begin{array}{llllllllllllllllll}24 & 41 & -19 & 19 & 25 & -5 & 23 & 27 & -10 & 15 & 0 & 0 & 18 & 1 & -2 & 23 & 1 & 4\end{array}$ $\begin{array}{lllllllllllllllllll}24 & 41 & -19 & 18 & 10 & 2 & 22 & 27 & -5 & 13 & 42 & 5 & 15 & 50 & 4 & 22 & 0 & 0\end{array}$ $\begin{array}{llllllllllllllllll}18 & 0 & 0 & 17 & 0 & 0 & 18 & 0 & 0 & 12 & 2 & -3 & 11 & 0 & 0 & 19 & 0 & 0\end{array}$ $\begin{array}{llllllllllllllllll}11 & 0 & 0 & 10 & 0 & 0 & 11 & 1 & 0 & 11 & 3 & 0 & 10 & 4 & 0 & 10 & 0 & 0\end{array}$ $\begin{array}{llllllllllllllllll}7 & 0 & 0 & 3 & 1 & 0 & 7 & 0 & 0 & -3 & 0 & 0 & 5 & 0 & 0 & 8 & 0 & 0\end{array}$

${ }^{\mathrm{a}} \mathrm{v}$ in $\mathrm{cm}^{-1}, \mathrm{D}$ in $10^{-40} \mathrm{esu}^{2} \mathrm{~cm}^{2}, \mathrm{R}$ in $10^{-44} \mathrm{esu}^{2} \mathrm{~cm}^{2}$ 
Table S4: B3LYP/6-31G* structural parameters of conformations aaaa, aaab and bbbb of $(\mathrm{S}-1)_{4}{ }^{\mathrm{a}}$.

\begin{tabular}{|c|c|c|c|c|c|}
\hline Bond Lengths & aaaa & $\mathbf{a a a b} \mathbf{b}^{\mathrm{b}}$ & $\mathbf{a} a \mathbf{a} \mathbf{b}^{\mathrm{c}}$ & $\mathbf{a a a b} \mathbf{b}^{\mathrm{d}}$ & bbbb \\
\hline $\mathrm{C} 1 \mathrm{O} 2$ & 1.234 & 1.234 & 1.234 & 1.236 & 1.236 \\
\hline $\mathrm{O} 2 \mathrm{H} 3$ & 1.680 & 1.681 & 1.682 & 1.670 & 1.671 \\
\hline $\mathrm{O} 2 \ldots \mathrm{O} 4$ & 2.683 & 2.684 & 2.685 & 2.676 & 2.675 \\
\hline H3O4 & 1.003 & 1.004 & 1.003 & 1.006 & 1.006 \\
\hline O4C5 & 1.327 & 1.327 & 1.327 & 1.322 & 1.321 \\
\hline $\mathrm{C} 1 \mathrm{C} 9$ & 1.490 & 1.490 & 1.490 & 1.493 & 1.494 \\
\hline \multicolumn{6}{|l|}{ Bond Angles } \\
\hline $\mathrm{C} 1 \mathrm{O} 2 \mathrm{H} 3$ & 125.8 & 125.9 & 125.8 & 125.5 & 125.4 \\
\hline $\mathrm{O} 2 \mathrm{H} 3 \mathrm{O} 4$ & 177.5 & 177.6 & 177.4 & 177.4 & 176.7 \\
\hline $\mathrm{H} 3 \mathrm{O} 4 \mathrm{C} 5$ & 109.6 & 109.6 & 109.6 & 109.7 & 109.8 \\
\hline O4C5O6 & 122.7 & 122.7 & 122.7 & 122.7 & 122.8 \\
\hline \multicolumn{6}{|l|}{ Dihedral Angles } \\
\hline H3O4C5O6 & 0.9 & 1.0 & 1.0 & -0.4 & -0.8 \\
\hline O4C5O6H7 & 1.0 & 1.6 & 0.8 & -1.1 & 0.5 \\
\hline $\mathrm{O} 2 \mathrm{O} 8 \mathrm{O} 6 \mathrm{O} 4$ & -2.5 & -3.1 & -2.6 & 2.0 & 1.2 \\
\hline $\mathrm{O} 2 \mathrm{C} 1 \mathrm{C} 9 \mathrm{C} 10$ & 5.2 & 5.8 & 5.2 & -177.0 & -175.2 \\
\hline O8C1C9C10 & -175.4 & -174.9 & -175.5 & 3.5 & 5.6 \\
\hline $\mathrm{C} 9 \mathrm{C} 10 \mathrm{C} 10^{\prime} \mathrm{C} 9^{\prime}{ }^{\text {e }}$ & 94.0 & 94.6 & 94.6 & 93.9 & 92.8 \\
\hline
\end{tabular}

${ }^{\text {a }}$ Bond lengths in $\AA$, bond angles and dihedrals in degrees. Atom numbering as in Table 1 .

${ }^{\mathrm{b}}$ Structural parameters are for one of the two equivalent $\mathbf{a}$ bridges $c i$ s to the $\mathbf{b}$ bridge.

${ }^{c}$ Structural parameters are for the $\mathbf{a}$ bridge trans to the $\mathbf{b}$ bridge.

${ }^{\mathrm{d}}$ Structural parameters are for the $\mathbf{b}$ bridge.

${ }^{\mathrm{e}} \mathrm{C} 9$ ' and $\mathrm{C} 10$ ' are the carbon atoms corresponding to $\mathrm{C} 9$ and $\mathrm{C} 10$ in the adjacent phenyl group of the biphenyl moiety. 


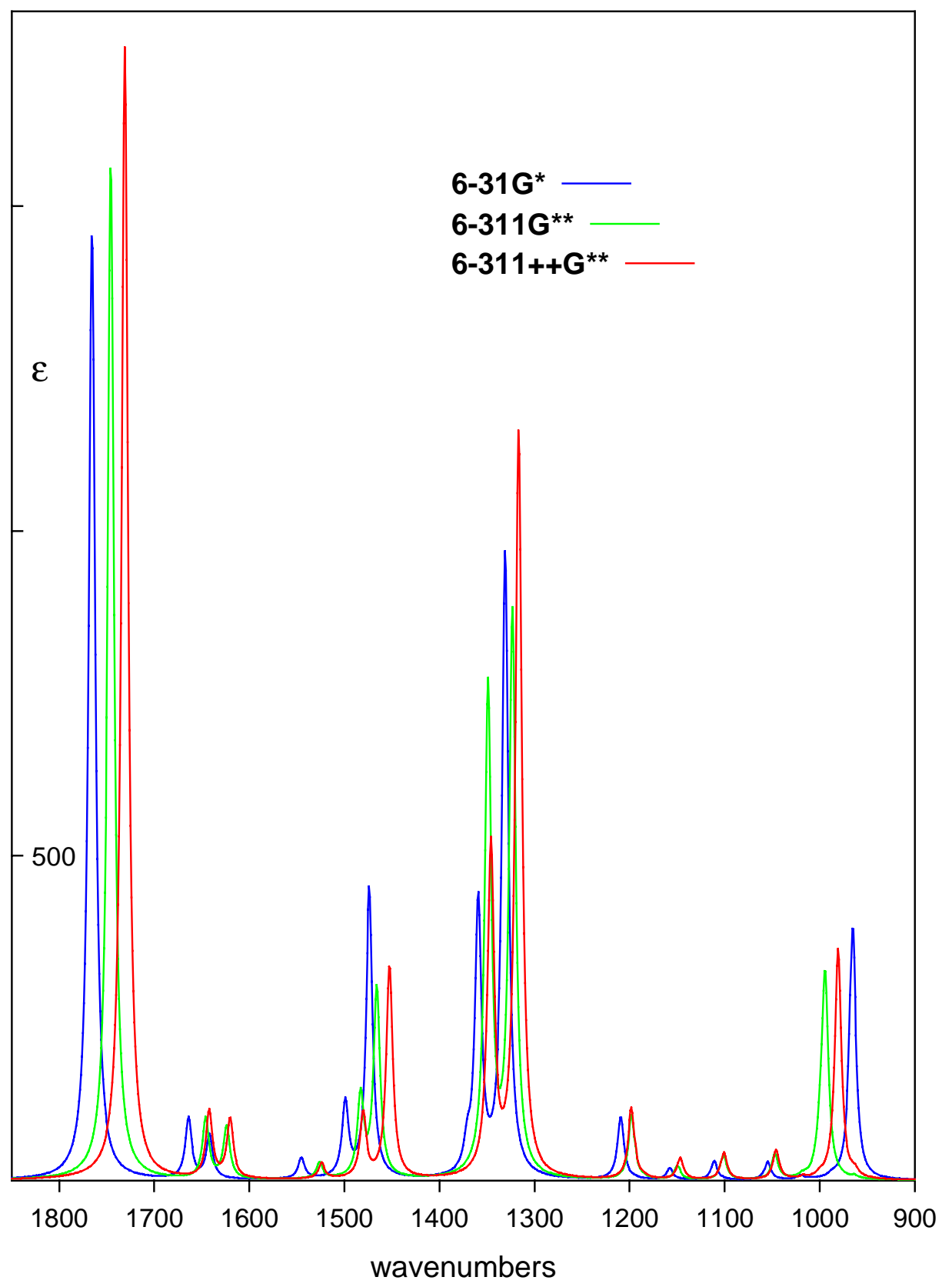

Fig. S1: Basis set dependence of the calculated IR spectrum of $(2)_{2}$. 


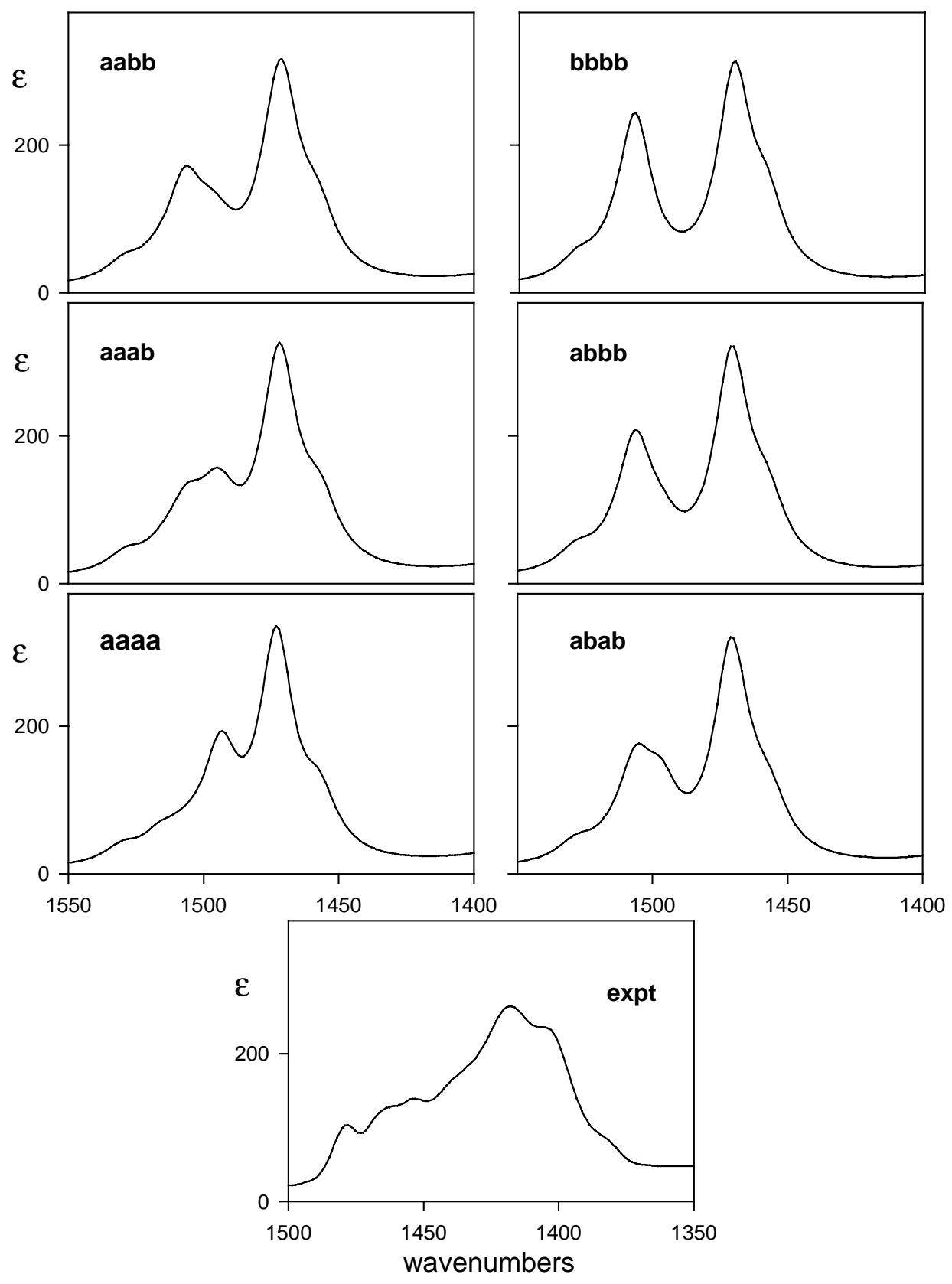

Fig. S2: B3LYP/6-31G* IR spectra of the six conformations of (S-1) 4 and the experimental IR spectrum $\left(\mathrm{CDCl}_{3} / 0.16 \mathrm{M}\right)$. The Lorentzian bandshape parameter $\gamma=7.5 \mathrm{~cm}^{-1}$ for all transitions. The dipole strengths of all transitions of aaab are plotted in Fig. S3. 

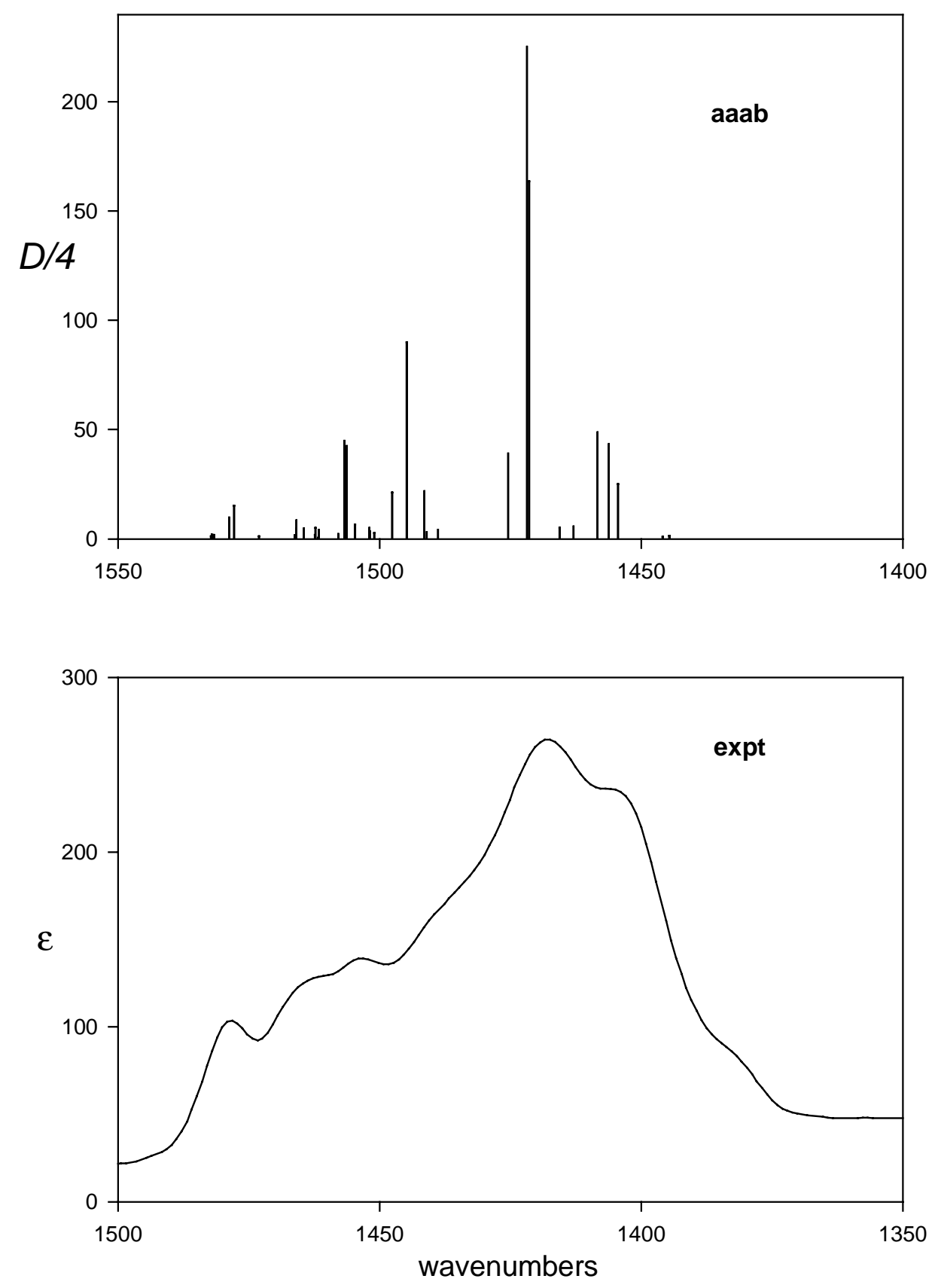

Fig. S3: Comparison of B3LYP/6-31G* frequencies and dipole strengths of conformation aaab of $(\mathrm{S}-\mathbf{1})_{4}$ and the experimental IR spectrum $\left(\mathrm{CDCl}_{3} / 0.16 \mathrm{M}\right)$. 


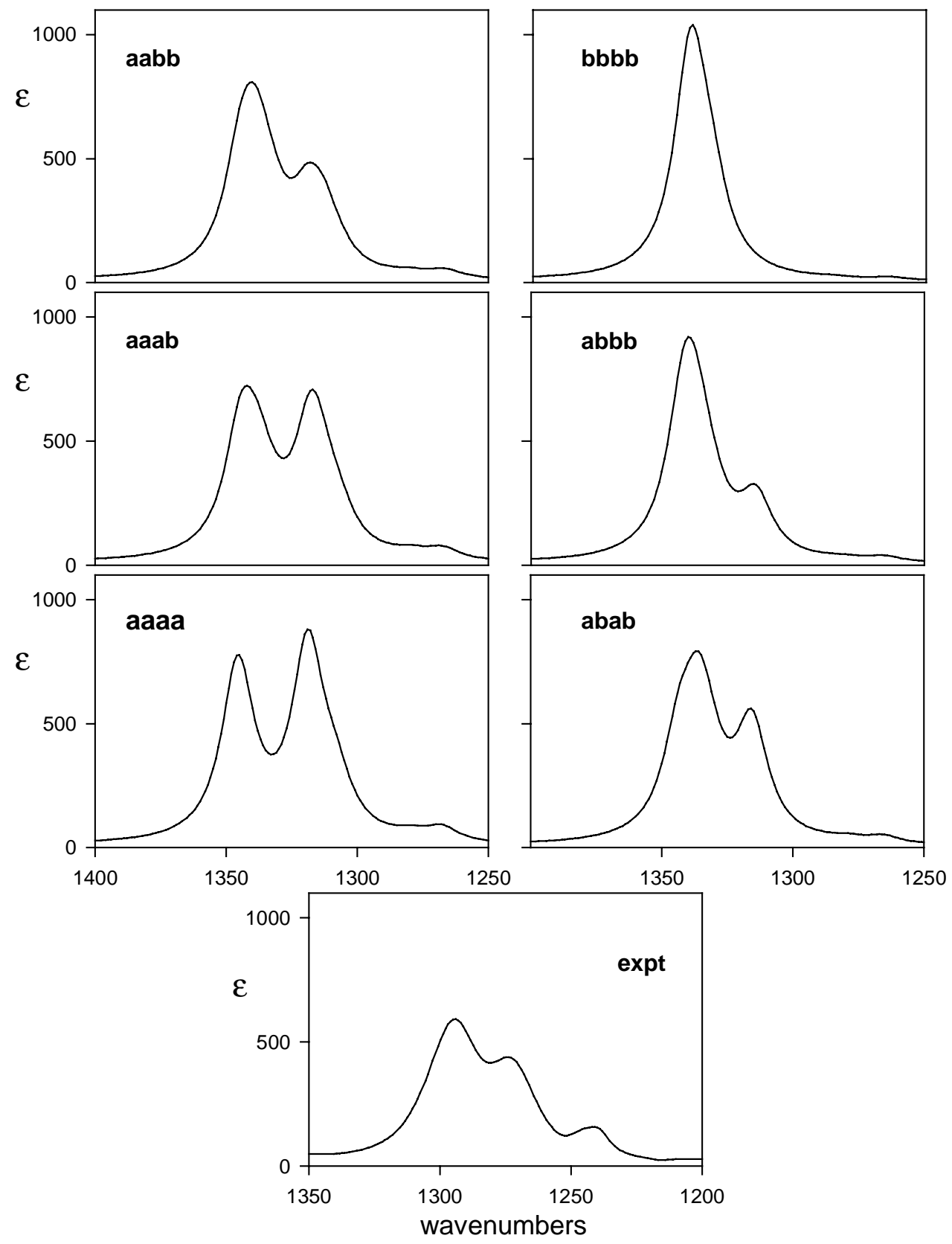

Fig. S4: B3LYP/6-31G* IR spectra of the six conformations of (S-1 $)_{4}$ and the experimental IR spectrum $\left(\mathrm{CDCl}_{3} / 0.16 \mathrm{M}\right)$. The Lorentzian bandshape parameter $\gamma=7.5 \mathrm{~cm}^{-1}$ for all transitions. The dipole strengths of all transitions of aaab are plotted in Fig. S5. 

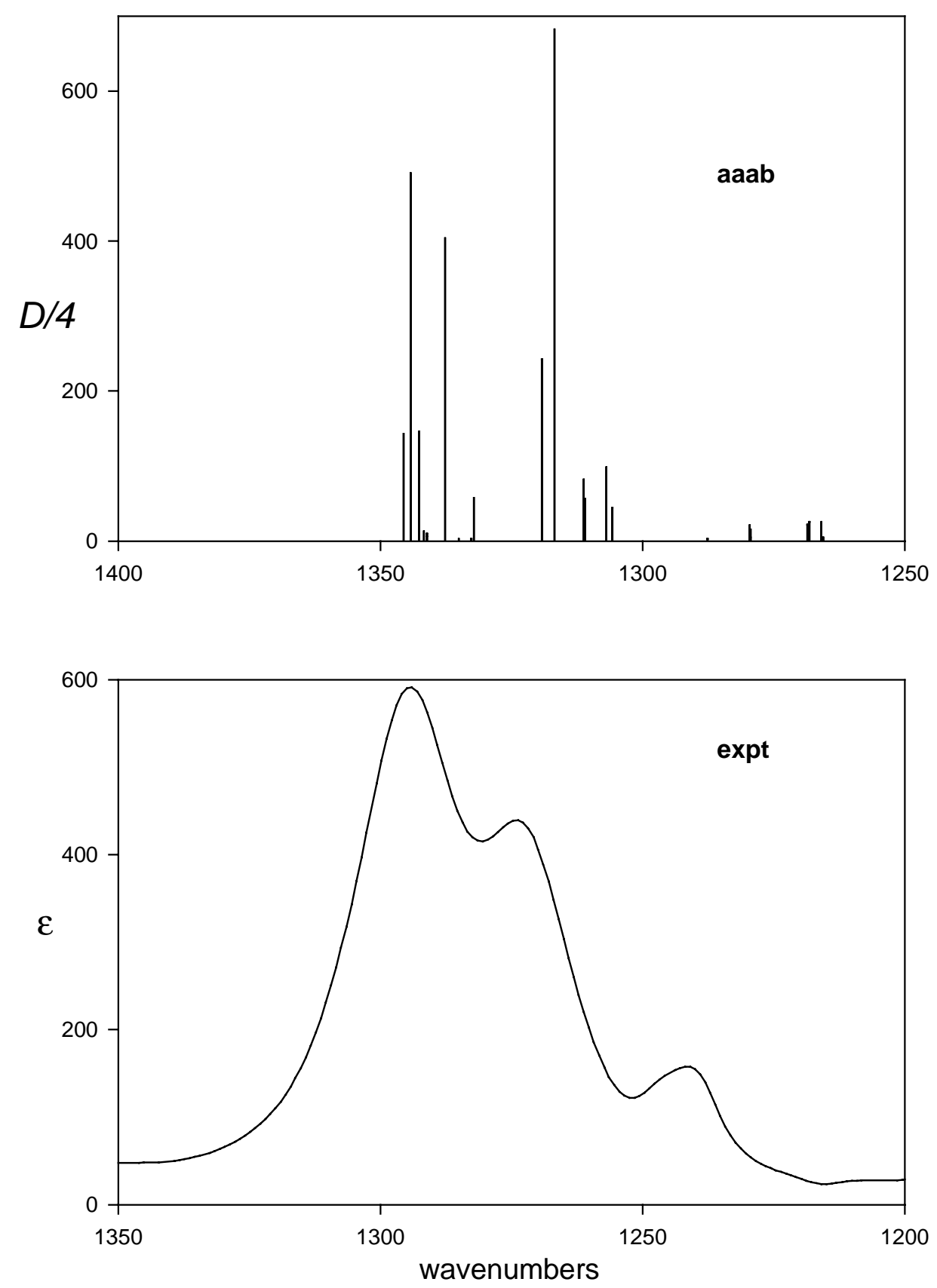

Fig. S5: Comparison of B3LYP/6-31G* frequencies and dipole strengths of conformation aaab of $(\mathrm{S}-1)_{4}$ and the experimental IR spectrum $\left(\mathrm{CDCl}_{3} / 0.16 \mathrm{M}\right)$. 


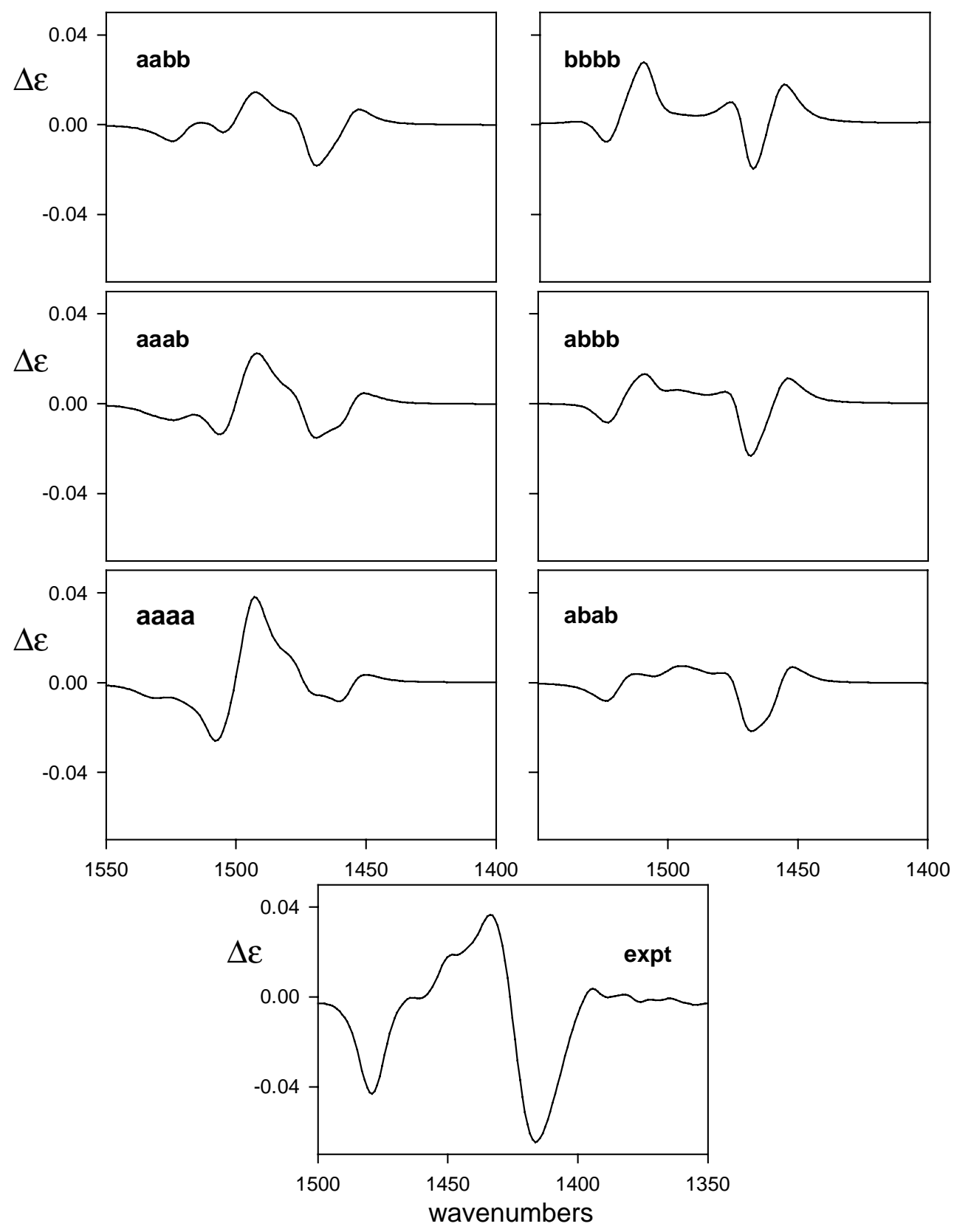

Fig. S6: B3LYP/6-31G* VCD spectra of the six conformations of $(\mathrm{S}-\mathbf{1})_{4}$ and the experimental VCD spectrum $\left(\mathrm{CDCl}_{3} / 0.16 \mathrm{M}\right)$. The Lorentzian bandshape parameter $\gamma=7.5 \mathrm{~cm}^{-1}$ for all transitions. The rotational strengths of all transitions of aaab are plotted in Fig. S7. 

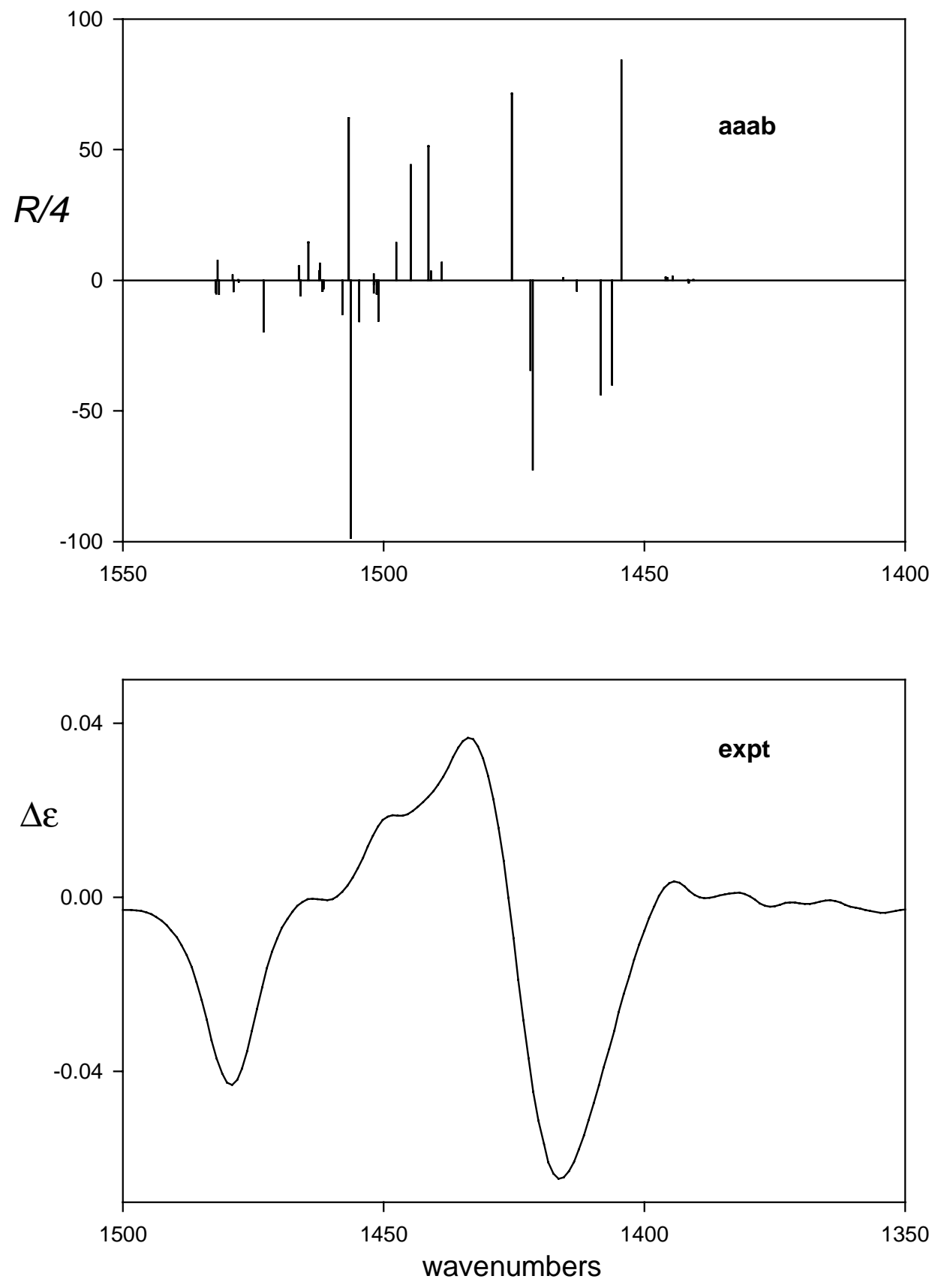

Fig. S7: Comparison of B3LYP/6-31G* frequencies and rotational strengths of conformation aaab of $(\mathrm{S}-1)_{4}$ and the experimental VCD spectrum $\left(\mathrm{CDCl}_{3} / 0.16 \mathrm{M}\right)$. 

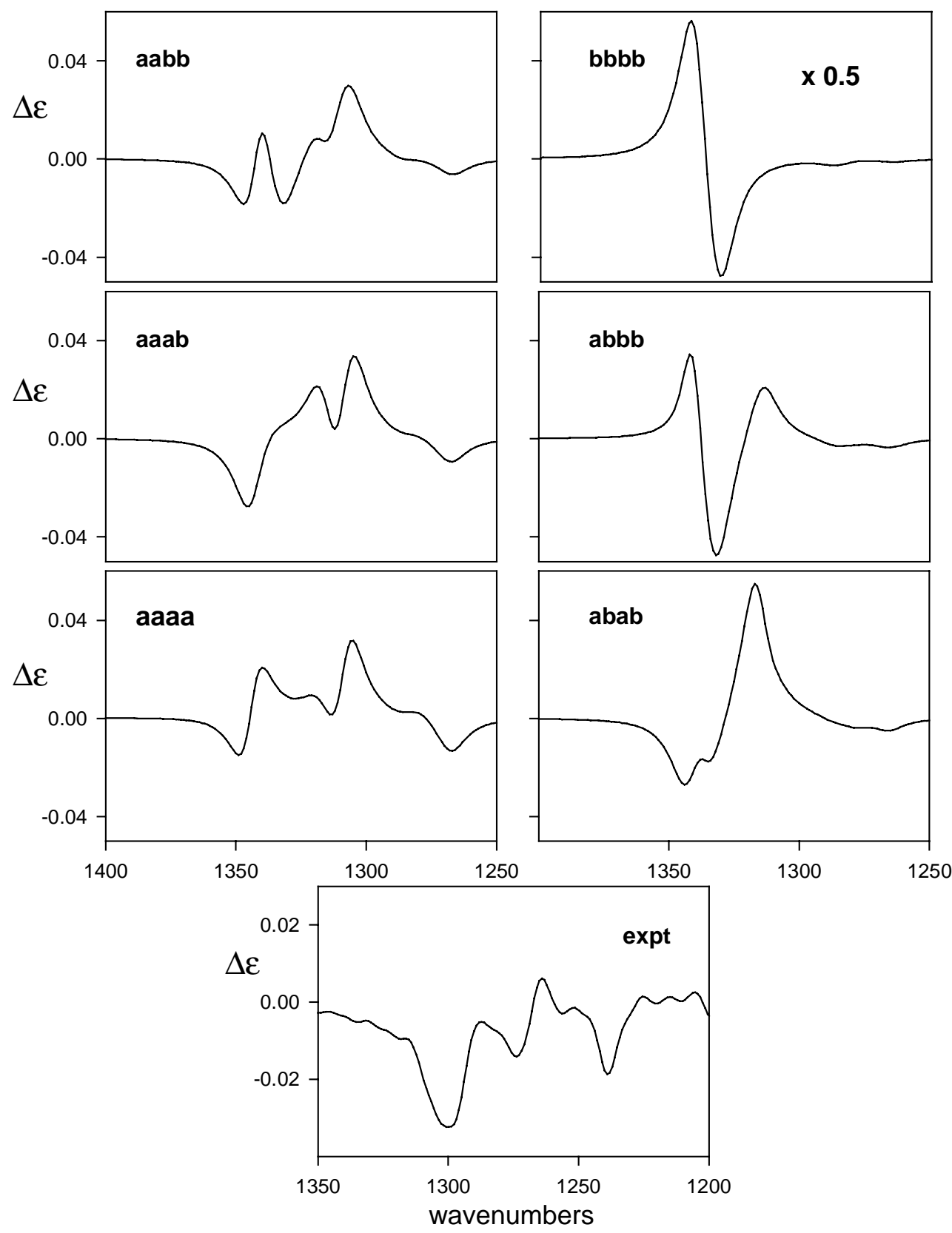

Fig. S8: B3LYP/6-31G* VCD spectra of the six conformations of $(\mathrm{S}-\mathbf{1})_{4}$ and the experimental VCD spectrum $\left(\mathrm{CDCl}_{3} / 0.16 \mathrm{M}\right)$. The Lorentzian bandshape parameter $\gamma=7.5 \mathrm{~cm}^{-1}$ for all transitions. The rotational strengths of all transitions of aaab are plotted in Fig. S9. 

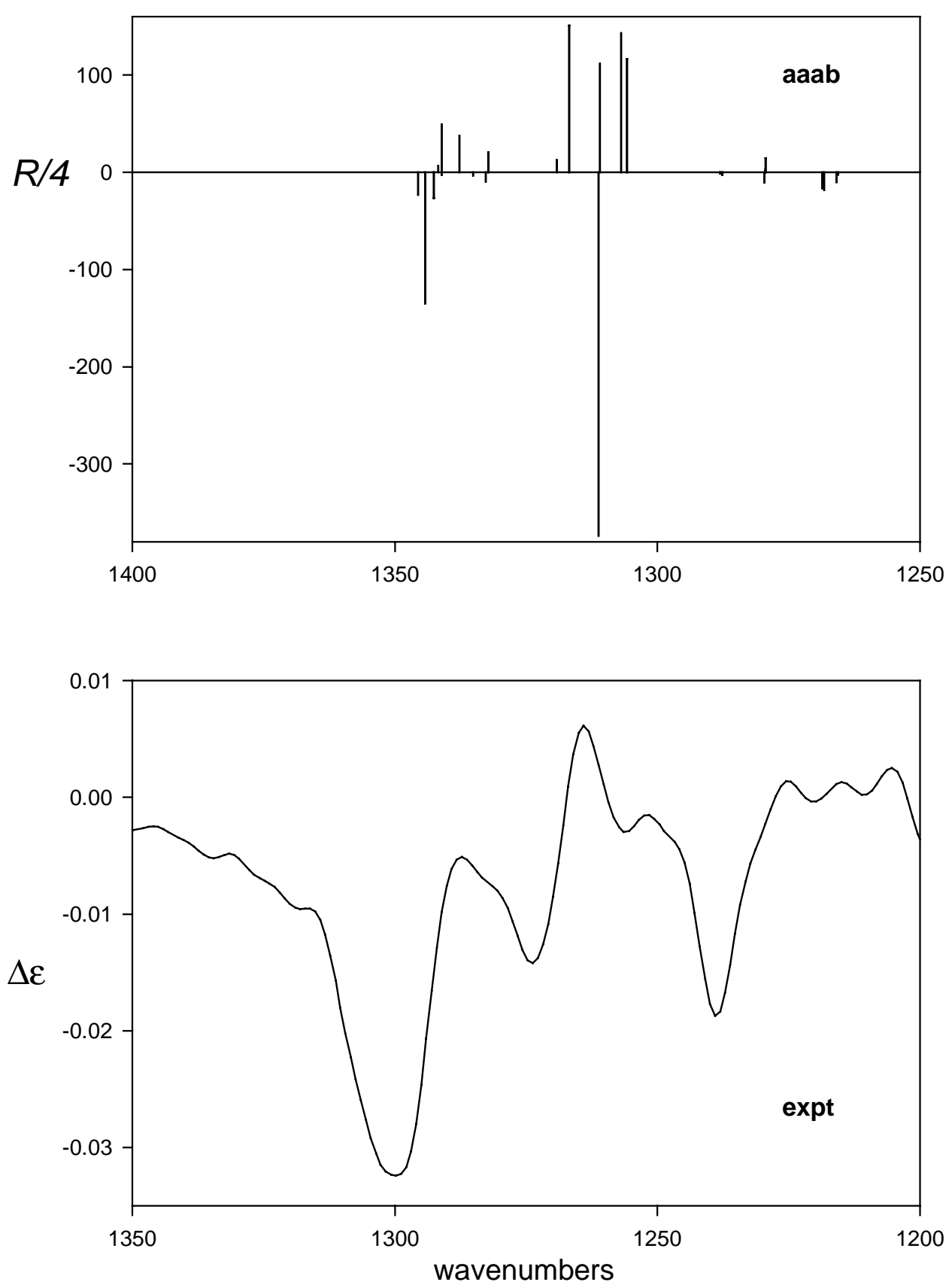

Fig. S9: Comparison of B3LYP/6-31G* frequencies and rotational strengths of conformation aaab of $(\mathrm{S}-1)_{4}$ and the experimental VCD spectrum $\left(\mathrm{CDCl}_{3} / 0.16 \mathrm{M}\right)$. 


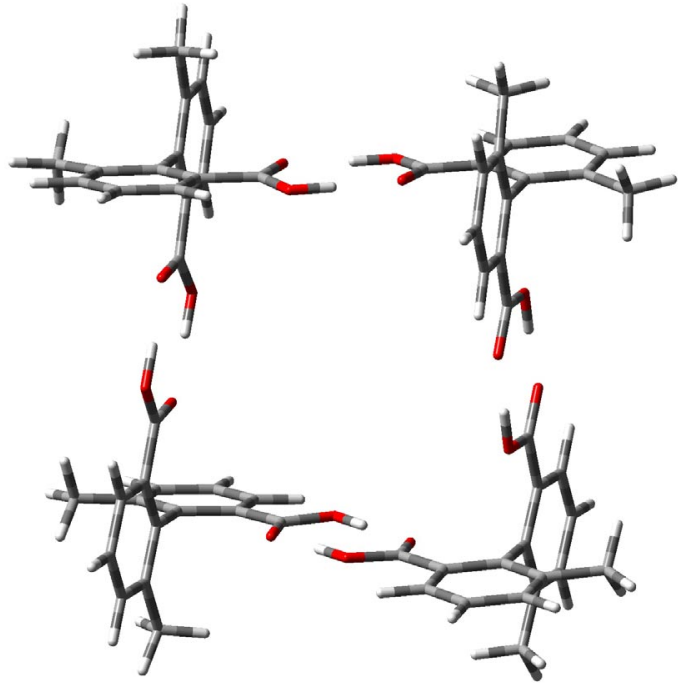

$(\mathrm{S}-1)_{4}:$ aaab (calc)
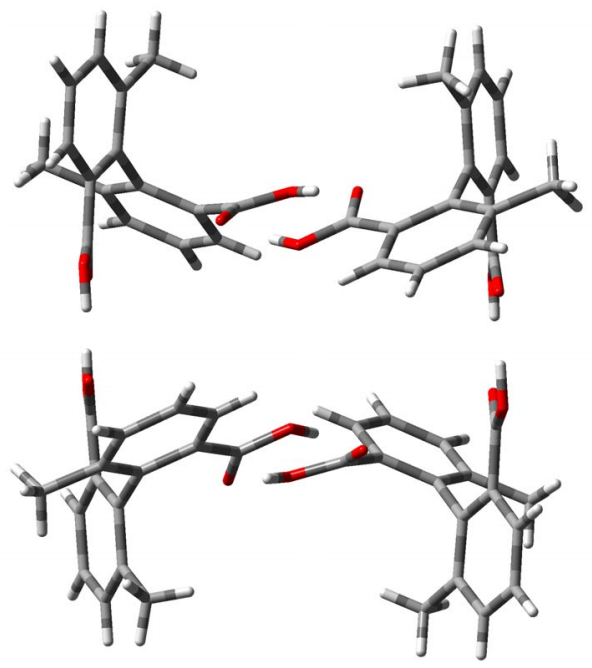

(R-1)4: aaaa (X-ray)

Fig. S10: The B3LYP/6-31G* structure of the aaab conformer of (S-1) $)_{4}$ and the X-ray structure of $(\mathrm{R}-\mathbf{1})_{4}$. 
$(\mathrm{S}-1)_{4}$ Conformer aaaa

B3LYP/6-31G* optimized geometry [E=-3676.40274446 hartrees] Cartesian coordinates (Angstroms)

\begin{tabular}{|c|c|c|c|}
\hline $\mathrm{O}$ & 2.51427400 & 3.55845500 & 1.69395500 \\
\hline C & 1.82109600 & 4.30398300 & 0.84305400 \\
\hline O & 1.93929000 & 4.20306600 & -0.38140900 \\
\hline $\mathrm{C}$ & 0.90382300 & 5.27602100 & 1.50089300 \\
\hline $\mathrm{C}$ & 0.01865100 & 6.08438800 & 0.75207900 \\
\hline $\mathrm{C}$ & -0.82912400 & 6.98541400 & 1.43391200 \\
\hline C & -0.77109500 & 7.05876600 & 2.82865000 \\
\hline C & 0.10001700 & 6.25789000 & 3.56528900 \\
\hline C & 0.93066600 & 5.36666400 & 2.90202200 \\
\hline $\mathrm{H}$ & 1.61186000 & 4.73080900 & 3.45400900 \\
\hline $\mathrm{H}$ & 0.12709100 & 6.32916300 & 4.64887800 \\
\hline $\mathrm{H}$ & -1.42820900 & 7.75533600 & 3.34432300 \\
\hline $\mathrm{C}$ & -1.82007600 & 7.84804700 & 0.68523300 \\
\hline $\mathrm{H}$ & -2.59385900 & 7.23337500 & 0.21018000 \\
\hline $\mathrm{H}$ & -1.34437200 & 8.43192700 & -0.11034300 \\
\hline $\mathrm{H}$ & -2.31837500 & 8.54471700 & 1.36620400 \\
\hline $\mathrm{C}$ & -0.01865100 & 6.08438800 & -0.75207900 \\
\hline C & -0.90382300 & 5.27602100 & -1.50089300 \\
\hline C & -1.82109600 & 4.30398300 & -0.84305400 \\
\hline O & -2.51427400 & 3.55845500 & -1.69395500 \\
\hline $\mathrm{H}$ & -3.12557000 & 2.94632500 & -1.18557800 \\
\hline O & -1.93929000 & 4.20306600 & 0.38140900 \\
\hline $\mathrm{C}$ & -0.93066600 & 5.36666400 & -2.90202200 \\
\hline $\mathrm{C}$ & -0.10001700 & 6.25789000 & -3.56528900 \\
\hline $\mathrm{C}$ & 0.77109500 & 7.05876600 & -2.82865000 \\
\hline $\mathrm{C}$ & 0.82912400 & 6.98541400 & -1.43391200 \\
\hline C & 1.82007600 & 7.84804700 & -0.68523300 \\
\hline $\mathrm{H}$ & 2.59385900 & 7.23337500 & -0.21018000 \\
\hline $\mathrm{H}$ & 1.34437200 & 8.43192700 & 0.11034300 \\
\hline $\mathrm{H}$ & 2.31837500 & 8.54471700 & -1.36620400 \\
\hline $\mathrm{H}$ & 1.42820900 & 7.75533600 & -3.34432300 \\
\hline $\mathrm{H}$ & -0.12709100 & 6.32916300 & -4.64887800 \\
\hline $\mathrm{H}$ & -1.61186000 & 4.73080900 & -3.45400900 \\
\hline $\mathrm{H}$ & 3.12557000 & 2.94632500 & 1.18557800 \\
\hline O & 3.55845500 & 2.51427400 & -1.69395500 \\
\hline $\mathrm{C}$ & 4.30398300 & 1.82109600 & -0.84305400 \\
\hline O & 4.20306600 & 1.93929000 & 0.38140900 \\
\hline $\mathrm{C}$ & 5.27602100 & 0.90382300 & -1.50089300 \\
\hline C & 6.08438800 & 0.01865100 & -0.75207900 \\
\hline C & 6.98541400 & -0.82912400 & -1.43391200 \\
\hline C & 7.05876600 & -0.77109500 & -2.82865000 \\
\hline $\mathrm{C}$ & 6.25789000 & 0.10001700 & -3.56528900 \\
\hline C & 5.36666400 & 0.93066600 & -2.90202200 \\
\hline $\mathrm{H}$ & 4.73080900 & 1.61186000 & -3.45400900 \\
\hline $\mathrm{H}$ & 6.32916300 & 0.12709100 & -4.64887800 \\
\hline $\mathrm{H}$ & 7.75533600 & -1.42820900 & -3.34432300 \\
\hline $\mathrm{C}$ & 7.84804700 & -1.82007600 & -0.68523300 \\
\hline $\mathrm{H}$ & 7.23337500 & -2.59385900 & -0.21018000 \\
\hline $\mathrm{H}$ & 8.43192700 & -1.34437200 & 0.11034300 \\
\hline $\mathrm{H}$ & 8.54471700 & -2.31837500 & -1.36620400 \\
\hline C & 6.08438800 & -0.01865100 & 0.75207900 \\
\hline $\mathrm{C}$ & 5.27602100 & -0.90382300 & 1.50089300 \\
\hline $\mathrm{C}$ & 4.30398300 & -1.82109600 & 0.84305400 \\
\hline O & 3.55845500 & -2.51427400 & 1.69395500 \\
\hline $\mathrm{H}$ & 2.94632500 & -3.12557000 & 1.18557800 \\
\hline O & 4.20306600 & -1.93929000 & -0.38140900 \\
\hline
\end{tabular}




\begin{tabular}{|c|c|c|c|}
\hline C & 5.36666400 & -0.93066600 & 2.90202200 \\
\hline & 6.25789000 & -0.10001700 & 3.56528900 \\
\hline & 7.05876600 & 0.77109500 & 2.82865000 \\
\hline & 6.98541400 & 0.82912400 & 1.43391200 \\
\hline$\gamma$ & 7.84804700 & 1.82007600 & 0.68523300 \\
\hline & 7.23337500 & 2.59385900 & 0.21018000 \\
\hline & 8.43192700 & 1.34437200 & -0.11034300 \\
\hline & 8.54471700 & 2.31837500 & 1.36620400 \\
\hline & 7.75533600 & 1.42820900 & 3.34432300 \\
\hline & 6.32916300 & -0.12709100 & 4.64887800 \\
\hline & 4.73080900 & -1.61186000 & 3.45400900 \\
\hline & 2.94632500 & 3.12557000 & -1.18557800 \\
\hline & -4.20306600 & 1.93929000 & -0.38140900 \\
\hline & -4.30398300 & 1.82109600 & 0.84305400 \\
\hline & -3.55845500 & 2.51427400 & 1.69395500 \\
\hline & -2.94632500 & 3.12557000 & 1.18557800 \\
\hline & -5.27602100 & 0.90382300 & 1.50089300 \\
\hline 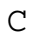 & -6.08438800 & 0.01865100 & 0.75207900 \\
\hline$C$ & -6.08438800 & -0.01865100 & -0.75207900 \\
\hline$C$ & -5.27602100 & -0.90382300 & -1.50089300 \\
\hline C & -4.30398300 & -1.82109600 & -0.84305400 \\
\hline o & -3.55845500 & -2.51427400 & -1.69395500 \\
\hline T & -2.94632500 & -3.12557000 & -1.18557800 \\
\hline O & -4.20306600 & -1.93929000 & 0.38140900 \\
\hline C & -5.36666400 & -0.93066600 & -2.90202200 \\
\hline C & -6.25789000 & -0.10001700 & -3.56528900 \\
\hline C & -7.05876600 & 0.77109500 & -2.82865000 \\
\hline C & -6.98541400 & 0.82912400 & -1.43391200 \\
\hline C & -7.84804700 & 1.82007600 & -0.68523300 \\
\hline $\mathrm{H}$ & -7.23337500 & 2.59385900 & -0.21018000 \\
\hline $\mathrm{H}$ & -8.43192700 & 1.34437200 & 0.11034300 \\
\hline $\mathrm{H}$ & -8.54471700 & 2.31837500 & -1.36620400 \\
\hline $\mathrm{H}$ & -7.75533600 & 1.42820900 & -3.34432300 \\
\hline $\mathrm{H}$ & -6.32916300 & -0.12709100 & -4.64887800 \\
\hline 世 & -4.73080900 & -1.61186000 & -3.45400900 \\
\hline C & -6.98541400 & -0.82912400 & 1.43391200 \\
\hline C & -7.05876600 & -0.77109500 & 2.82865000 \\
\hline C & -6.25789000 & 0.10001700 & 3.56528900 \\
\hline C & -5.36666400 & 0.93066600 & 2.90202200 \\
\hline $\mathrm{H}$ & -4.73080900 & 1.61186000 & 3.45400900 \\
\hline $\mathrm{H}$ & -6.32916300 & 0.12709100 & 4.64887800 \\
\hline $\mathrm{H}$ & -7.75533600 & -1.42820900 & 3.34432300 \\
\hline C & -7.84804700 & -1.82007600 & 0.68523300 \\
\hline $\mathrm{H}$ & -7.23337500 & -2.59385900 & 0.21018000 \\
\hline $\mathrm{H}$ & -8.43192700 & -1.34437200 & -0.11034300 \\
\hline $\mathrm{H}$ & -8.54471700 & -2.31837500 & 1.36620400 \\
\hline 0 & -2.51427400 & -3.55845500 & 1.69395500 \\
\hline C & -1.82109600 & -4.30398300 & 0.84305400 \\
\hline 0 & -1.93929000 & -4.20306600 & -0.38140900 \\
\hline C & -0.90382300 & -5.27602100 & 1.50089300 \\
\hline C & -0.01865100 & -6.08438800 & 0.75207900 \\
\hline C & 0.82912400 & -6.98541400 & 1.43391200 \\
\hline C & 0.77109500 & -7.05876600 & 2.82865000 \\
\hline C & -0.10001700 & -6.25789000 & 3.56528900 \\
\hline C & -0.93066600 & -5.36666400 & 2.90202200 \\
\hline $\mathrm{H}$ & -1.61186000 & -4.73080900 & 3.45400900 \\
\hline $\mathrm{H}$ & -0.12709100 & -6.32916300 & 4.64887800 \\
\hline $\mathrm{H}$ & 1.42820900 & -7.75533600 & 3.34432300 \\
\hline C & 1.82007600 & -7.84804700 & 0.68523300 \\
\hline & 2.59385900 & -7.23337500 & 0.21018000 \\
\hline
\end{tabular}




$\begin{array}{lrrr}\mathrm{H} & 1.34437200 & -8.43192700 & -0.11034300 \\ \mathrm{H} & 2.31837500 & -8.54471700 & 1.36620400 \\ \mathrm{C} & 0.01865100 & -6.08438800 & -0.75207900 \\ \mathrm{C} & 0.90382300 & -5.27602100 & -1.50089300 \\ \mathrm{C} & 1.82109600 & -4.30398300 & -0.84305400 \\ \mathrm{O} & 2.51427400 & -3.55845500 & -1.69395500 \\ \mathrm{H} & 3.12557000 & -2.94632500 & -1.18557800 \\ \mathrm{O} & 1.93929000 & -4.20306600 & 0.38140900 \\ \mathrm{C} & 0.93066600 & -5.36666400 & -2.90202200 \\ \mathrm{C} & 0.10001700 & -6.25789000 & -3.56528900 \\ \mathrm{C} & -0.77109500 & -7.05876600 & -2.82865000 \\ \mathrm{C} & -0.82912400 & -6.98541400 & -1.43391200 \\ \mathrm{C} & -1.82007600 & -7.84804700 & -0.68523300 \\ \mathrm{H} & -2.59385900 & -7.23337500 & -0.21018000 \\ \mathrm{H} & -1.34437200 & -8.43192700 & 0.11034300 \\ \mathrm{H} & -2.31837500 & -8.54471700 & -1.36620400 \\ \mathrm{H} & -1.42820900 & -7.75533600 & -3.34432300 \\ \mathrm{H} & 0.12709100 & -6.32916300 & -4.64887800 \\ \mathrm{H} & 1.61186000 & -4.73080900 & -3.45400900 \\ \mathrm{H} & -3.12557000 & -2.94632500 & 1.18557800\end{array}$

$(\mathrm{S}-1)_{4}$ conformer aaab B3LYP/6-31G* optimized geometry [E=-3676.40095337 hartrees ] Cartesian coordinates (Angstroms)

$\begin{array}{rrrr}\mathrm{O} & 1.68679200 & 0.75741600 & -4.28547600 \\ \mathrm{C} & 0.82501500 & 1.76560100 & -4.32497700 \\ \mathrm{O} & -0.39765700 & 1.59697200 & -4.33565500 \\ \mathrm{C} & 1.46831000 & 3.10879800 & -4.36995600 \\ \mathrm{C} & 0.70657100 & 4.29826200 & -4.32046000 \\ \mathrm{C} & 1.37398500 & 5.54246800 & -4.37038100 \\ \mathrm{C} & 2.76800700 & 5.56811400 & -4.46898000 \\ \mathrm{C} & 3.51776700 & 4.39374300 & -4.51112400 \\ \mathrm{C} & 2.86880400 & 3.16897700 & -4.45742800 \\ \mathrm{H} & 3.43155000 & 2.24394500 & -4.48449500 \\ \mathrm{H} & 4.60074000 & 4.43686500 & -4.58415700 \\ \mathrm{H} & 3.27326400 & 6.53049400 & -4.50685600 \\ \mathrm{C} & 0.61061800 & 6.84459900 & -4.28327600 \\ \mathrm{H} & 0.14766100 & 6.95966800 & -3.29603900 \\ \mathrm{H} & -0.19599000 & 6.90383700 & -5.02207500 \\ \mathrm{H} & 1.27878500 & 7.69685500 & -4.44024400 \\ \mathrm{C} & -0.79776400 & 4.30746800 & -4.28739300 \\ \mathrm{C} & -1.54139400 & 4.37424700 & -3.08739000 \\ \mathrm{C} & -0.87649200 & 4.37506600 & -1.75414600 \\ \mathrm{O} & -1.72132300 & 4.33345000 & -0.73184100 \\ \mathrm{H} & -1.20888400 & 4.36371200 & 0.12986700 \\ \mathrm{O} & 0.34791900 & 4.42292000 & -1.60509200 \\ \mathrm{C} & -2.94396800 & 4.43555900 & -3.12695200 \\ \mathrm{C} & -3.61313400 & 4.44712800 & -4.34195200 \\ \mathrm{C} & -2.88139500 & 4.38828000 & -5.52688200 \\ \mathrm{C} & -1.48560000 & 4.31421500 & -5.52138600 \\ \mathrm{C} & -0.74157500 & 4.20741700 & -6.83326900 \\ \mathrm{H} & -0.26311100 & 3.22575200 & -6.93159000 \\ \mathrm{H} & 0.05091300 & 4.95820500 & -6.92397100 \\ \mathrm{H} & -1.42587300 & 4.33144900 & -7.67811300 \\ \mathrm{H} & -3.40240500 & 4.39292300 & -6.48156800 \\ \mathrm{H} & -4.69776800 & 4.50036500 & -4.36924200 \\ \mathrm{H} & -3.49243900 & 4.47627400 & -2.19387600 \\ \mathrm{H} & 1.18744500 & -0.11268800 & -4.28346700\end{array}$




\begin{tabular}{|c|c|c|c|}
\hline O & -1.68679200 & -0.75741600 & -4.28547600 \\
\hline $\mathrm{C}$ & -0.82501500 & -1.76560100 & -4.32497700 \\
\hline O & 0.39765700 & -1.59697200 & -4.33565500 \\
\hline $\mathrm{C}$ & -1.46831000 & -3.10879800 & -4.36995600 \\
\hline $\mathrm{C}$ & -0.70657100 & -4.29826200 & -4.32046000 \\
\hline $\mathrm{C}$ & -1.37398500 & -5.54246800 & -4.37038100 \\
\hline $\mathrm{C}$ & -2.76800700 & -5.56811400 & -4.46898000 \\
\hline $\mathrm{C}$ & -3.51776700 & -4.39374300 & -4.51112400 \\
\hline $\mathrm{C}$ & -2.86880400 & -3.16897700 & -4.45742800 \\
\hline $\mathrm{H}$ & -3.43155000 & -2.24394500 & -4.48449500 \\
\hline $\mathrm{H}$ & -4.60074000 & -4.43686500 & -4.58415700 \\
\hline $\mathrm{H}$ & -3.27326400 & -6.53049400 & -4.50685600 \\
\hline $\mathrm{C}$ & -0.61061800 & -6.84459900 & -4.28327600 \\
\hline $\mathrm{H}$ & -0.14766100 & -6.95966800 & -3.29603900 \\
\hline $\mathrm{H}$ & 0.19599000 & -6.90383700 & -5.02207500 \\
\hline $\mathrm{H}$ & -1.27878500 & -7.69685500 & -4.44024400 \\
\hline $\mathrm{C}$ & 0.79776400 & -4.30746800 & -4.28739300 \\
\hline $\mathrm{C}$ & 1.54139400 & -4.37424700 & -3.08739000 \\
\hline $\mathrm{C}$ & 0.87649200 & -4.37506600 & -1.75414600 \\
\hline O & 1.72132300 & -4.33345000 & -0.73184100 \\
\hline $\mathrm{H}$ & 1.20888400 & -4.36371200 & 0.12986700 \\
\hline O & -0.34791900 & -4.42292000 & -1.60509200 \\
\hline $\mathrm{C}$ & 2.94396800 & -4.43555900 & -3.12695200 \\
\hline $\mathrm{C}$ & 3.61313400 & -4.44712800 & -4.34195200 \\
\hline $\mathrm{C}$ & 2.88139500 & -4.38828000 & -5.52688200 \\
\hline $\mathrm{C}$ & 1.48560000 & -4.31421500 & -5.52138600 \\
\hline $\mathrm{C}$ & 0.74157500 & -4.20741700 & -6.83326900 \\
\hline $\mathrm{H}$ & 0.26311100 & -3.22575200 & -6.93159000 \\
\hline $\mathrm{H}$ & -0.05091300 & -4.95820500 & -6.92397100 \\
\hline $\mathrm{H}$ & 1.42587300 & -4.33144900 & -7.67811300 \\
\hline $\mathrm{H}$ & 3.40240500 & -4.39292300 & -6.48156800 \\
\hline $\mathrm{H}$ & 4.69776800 & -4.50036500 & -4.36924200 \\
\hline $\mathrm{H}$ & 3.49243900 & -4.47627400 & -2.19387600 \\
\hline $\mathrm{H}$ & -1.18744500 & 0.11268800 & -4.28346700 \\
\hline 0 & -0.39640800 & 4.46495000 & 1.59950900 \\
\hline $\mathrm{C}$ & 0.82860800 & 4.47369800 & 1.74826900 \\
\hline 0 & 1.67525600 & 4.43264600 & 0.72772500 \\
\hline $\mathrm{H}$ & 1.16242800 & 4.41149900 & -0.13454900 \\
\hline $\mathrm{C}$ & 1.49194800 & 4.54623100 & 3.08096500 \\
\hline $\mathrm{C}$ & 0.75263100 & 4.46311300 & 4.28256300 \\
\hline $\mathrm{C}$ & -0.74767000 & 4.35255000 & 4.31732300 \\
\hline $\mathrm{C}$ & -1.43876900 & 3.12095900 & 4.38749900 \\
\hline $\mathrm{C}$ & -0.79189500 & 1.77549000 & 4.35669900 \\
\hline O & 0.52943400 & 1.76451200 & 4.32866500 \\
\hline $\mathrm{H}$ & 0.86084200 & 0.81491700 & 4.32596700 \\
\hline O & -1.46831000 & 0.74056000 & 4.36830100 \\
\hline $\mathrm{C}$ & -2.84020400 & 3.10361000 & 4.47888500 \\
\hline $\mathrm{C}$ & -3.56223800 & 4.28606600 & 4.51524800 \\
\hline $\mathrm{C}$ & -2.88288300 & 5.50183800 & 4.45118700 \\
\hline $\mathrm{C}$ & -1.49040000 & 5.55551100 & 4.34907900 \\
\hline C & -0.80632400 & 6.89953700 & 4.23792600 \\
\hline $\mathrm{H}$ & -0.34308700 & 7.02062800 & 3.25161100 \\
\hline $\mathrm{H}$ & -0.01110700 & 7.02473900 & 4.98094000 \\
\hline $\mathrm{H}$ & -1.52627100 & 7.71277900 & 4.37101500 \\
\hline $\mathrm{H}$ & -3.44310500 & 6.43377900 & 4.47345100 \\
\hline $\mathrm{H}$ & -4.64572300 & 4.26568900 & 4.59058400 \\
\hline $\mathrm{H}$ & -3.33641500 & 2.14101900 & 4.52046600 \\
\hline $\mathrm{C}$ & 1.43739300 & 4.53812000 & 5.51563700 \\
\hline $\mathrm{C}$ & 2.82679500 & 4.69554300 & 5.51884800 \\
\hline $\mathrm{C}$ & 3.55417500 & 4.77200500 & 4.33262400 \\
\hline
\end{tabular}




$\begin{array}{lrrr}\mathrm{C} & 2.88786100 & 4.69302300 & 3.11816400 \\ \mathrm{H} & 3.43365600 & 4.74461200 & 2.18402800 \\ \mathrm{H} & 4.63365900 & 4.89067000 & 4.35803300 \\ \mathrm{H} & 3.34583300 & 4.75229900 & 6.47287800 \\ \mathrm{C} & 0.70001100 & 4.41951000 & 6.83052700 \\ \mathrm{H} & 0.27286100 & 3.41709200 & 6.95263300 \\ \mathrm{H} & -0.13116000 & 5.12913600 & 6.90520400 \\ \mathrm{H} & 1.37676600 & 4.59856700 & 7.67152200 \\ \mathrm{O} & -0.52943400 & -1.76451200 & 4.32866500 \\ \mathrm{C} & 0.79189500 & -1.77549000 & 4.35669900 \\ \mathrm{O} & 1.46831000 & -0.74056000 & 4.36830100 \\ \mathrm{C} & 1.43876900 & -3.12095900 & 4.38749900 \\ \mathrm{C} & 0.74767000 & -4.35255000 & 4.31732300 \\ \mathrm{C} & 1.49040000 & -5.55551100 & 4.34907900 \\ \mathrm{C} & 2.88288300 & -5.50183800 & 4.45118700 \\ \mathrm{C} & 3.56223800 & -4.28606600 & 4.51524800 \\ \mathrm{C} & 2.84020400 & -3.10361000 & 4.47888500 \\ \mathrm{H} & 3.33641500 & -2.14101900 & 4.52046600 \\ \mathrm{H} & 4.64572300 & -4.26568900 & 4.59058400 \\ \mathrm{H} & 3.44310500 & -6.43377900 & 4.47345100 \\ \mathrm{C} & 0.80632400 & -6.89953700 & 4.23792600 \\ \mathrm{H} & 0.34308700 & -7.02062800 & 3.25161100 \\ \mathrm{H} & 0.01110700 & -7.02473900 & 4.98094000 \\ \mathrm{H} & 1.52627100 & -7.71277900 & 4.37101500 \\ \mathrm{C} & -0.75263100 & -4.46311300 & 4.28256300 \\ \mathrm{C} & -1.49194800 & -4.54623100 & 3.08096500 \\ \mathrm{C} & -0.82860800 & -4.47369800 & 1.74826900 \\ \mathrm{O} & -1.67525600 & -4.43264600 & 0.72772500 \\ \mathrm{H} & -1.16242800 & -4.41149900 & -0.13454900 \\ \mathrm{O} & 0.39640800 & -4.46495000 & 1.59950900 \\ \mathrm{C} & -2.88786100 & -4.69302300 & 3.11816400 \\ \mathrm{C} & -3.55417500 & -4.77200500 & 4.33262400 \\ \mathrm{C} & -2.82679500 & -4.69554300 & 5.51884800 \\ \mathrm{C} & -1.43739300 & -4.53812000 & 5.51563700 \\ \mathrm{C} & -0.70001100 & -4.41951000 & 6.83052700 \\ \mathrm{H} & -0.27286100 & -3.41709200 & 6.95263300 \\ \mathrm{H} & 0.13116000 & -5.12913600 & 6.90520400 \\ \mathrm{H} & -1.37676600 & -4.59856700 & 7.67152200 \\ \mathrm{H} & -3.34583300 & -4.75229900 & 6.47287800 \\ \mathrm{H} & -4.63365900 & -4.89067000 & 4.35803300 \\ \mathrm{H} & -3.43365600 & -4.74461200 & 2.18402800 \\ \mathrm{H} & -0.86084200 & -0.81491700 & 4.32596700\end{array}$

$(\mathrm{S}-1)_{4}$ conformer aabb

B3LYP/6-31G* optimized geometry [E=-3676.39962855 hartrees] Cartesian coordinates (Angstroms)

$\begin{array}{lrrr}\mathrm{O} & 3.62218000 & -2.42135700 & 1.66409900 \\ \mathrm{C} & 4.36968900 & -1.72147900 & 0.82035600 \\ \mathrm{O} & 4.29993400 & -1.85943000 & -0.40426600 \\ \mathrm{C} & 5.30601700 & -0.77374700 & 1.48700600 \\ \mathrm{C} & 6.08939600 & 0.14035600 & 0.74640300 \\ \mathrm{C} & 6.96524500 & 1.00801500 & 1.43611600 \\ \mathrm{C} & 7.03794800 & 0.94167400 & 2.83048900 \\ \mathrm{C} & 6.25952200 & 0.04401700 & 3.55926000 \\ \mathrm{C} & 5.39358100 & -0.80693500 & 2.88825300 \\ \mathrm{H} & 4.77681100 & -1.51022400 & 3.43416500 \\ \mathrm{H} & 6.32926400 & 0.01137800 & 4.64279900 \\ \mathrm{H} & 7.71540200 & 1.61375000 & 3.35229900\end{array}$




\begin{tabular}{|c|c|c|c|}
\hline C & 7.80080800 & 2.02845800 & 0.69648200 \\
\hline $\mathrm{H}$ & 7.16558600 & 2.79088400 & 0.23015300 \\
\hline $\mathrm{H}$ & 8.39538500 & 1.57610000 & -0.10469400 \\
\hline $\mathrm{H}$ & 8.48536300 & 2.53776600 & 1.38157700 \\
\hline $\mathrm{C}$ & 6.08608800 & 0.19292100 & -0.75722000 \\
\hline C & 5.25479500 & 1.06596900 & -1.49520000 \\
\hline C & 4.26560600 & 1.95726100 & -0.82667200 \\
\hline & 3.50410300 & 2.64269100 & -1.66997200 \\
\hline & 2.88565900 & 3.24139800 & -1.15512200 \\
\hline 0 & 4.16544000 & 2.06385600 & 0.39888800 \\
\hline C & 5.34194400 & 1.10903700 & -2.89644000 \\
\hline C & 6.25185900 & 0.30730300 & -3.56961700 \\
\hline C & 7.07598700 & -0.55088600 & -2.84332900 \\
\hline C & 7.00696100 & -0.62483700 & -1.44934300 \\
\hline C & 7.89426400 & -1.60191900 & -0.71174100 \\
\hline $\mathrm{H}$ & 7.29813900 & -2.39572400 & -0.24633300 \\
\hline $\mathrm{H}$ & 8.46577800 & -1.12087500 & 0.08959400 \\
\hline $\mathrm{H}$ & 8.60348700 & -2.07510000 & -1.39771300 \\
\hline $\mathrm{H}$ & 7.78761500 & -1.18507500 & -3.36703600 \\
\hline $\mathrm{H}$ & 6.32015600 & 0.34707100 & -4.65301100 \\
\hline $\mathrm{H}$ & 4.68898100 & 1.78039100 & -3.44042000 \\
\hline $\mathrm{H}$ & 3.04235800 & -3.05924900 & 1.15136600 \\
\hline 0 & 2.70987400 & -3.56547500 & -1.73261300 \\
\hline C & 2.02417900 & -4.32333500 & -0.88650400 \\
\hline 0 & 2.09991200 & -4.19221500 & 0.33807800 \\
\hline $\mathrm{C}$ & 1.17125800 & -5.34946200 & -1.55075200 \\
\hline C & 0.29213700 & -6.17443800 & -0.81301800 \\
\hline C & -0.49368500 & -7.12614900 & -1.49984200 \\
\hline C & -0.38146300 & -7.23261900 & -2.88945700 \\
\hline C & 0.48457800 & -6.41669900 & -3.61506100 \\
\hline C & 1.25448000 & -5.47562300 & -2.94678000 \\
\hline $\mathrm{H}$ & 1.92991500 & -4.82715900 & -3.49115400 \\
\hline $\mathrm{H}$ & 0.55479900 & -6.51456300 & -4.69463500 \\
\hline $\mathrm{H}$ & -0.99116400 & -7.96773100 & -3.40970100 \\
\hline C & -1.47461700 & -8.01052800 & -0.76327700 \\
\hline $\mathrm{H}$ & -2.28379900 & -7.41769400 & -0.32079700 \\
\hline $\mathrm{H}$ & -1.00082700 & -8.56175600 & 0.05646000 \\
\hline $\mathrm{H}$ & -1.92725500 & -8.73761800 & -1.44433800 \\
\hline C & 0.19313800 & -6.13221700 & 0.68783200 \\
\hline C & -0.75048300 & -5.34508500 & 1.38739500 \\
\hline C & -1.71858400 & -4.40520200 & 0.74843400 \\
\hline 0 & -1.72228600 & -4.38245500 & -0.57309600 \\
\hline $\mathrm{H}$ & -2.42134700 & -3.73769300 & -0.89960100 \\
\hline 0 & -2.47608300 & -3.70558800 & 1.43058000 \\
\hline C & -0.81752500 & -5.40496100 & 2.78885600 \\
\hline C & 0.02503900 & -6.24240100 & 3.50300100 \\
\hline C & 0.95227300 & -7.02396800 & 2.81532400 \\
\hline C & 1.05352600 & -6.98088100 & 1.42226900 \\
\hline C & 2.10421600 & -7.81919200 & 0.72905600 \\
\hline $\mathrm{H}$ & 2.86385800 & -7.18453800 & 0.25759000 \\
\hline $\mathrm{H}$ & 1.67939200 & -8.44744500 & -0.06145100 \\
\hline $\mathrm{H}$ & 2.61245100 & -8.47229900 & 1.44500600 \\
\hline $\mathrm{H}$ & 1.62004900 & -7.67978900 & 3.36922200 \\
\hline $\mathrm{H}$ & -0.03527900 & -6.28779000 & 4.58665500 \\
\hline $\mathrm{H}$ & -1.54635000 & -4.77994800 & 3.29159700 \\
\hline $\mathrm{H}$ & 3.28056400 & -2.91907100 & -1.21948200 \\
\hline 0 & 1.87758800 & 4.31137700 & -0.33625200 \\
\hline C & 1.77640800 & 4.41520900 & 0.88916200 \\
\hline 0 & 2.47893900 & 3.67022300 & 1.73268200 \\
\hline $\mathrm{H}$ & 3.08596200 & 3.05929300 & 1.21772900 \\
\hline
\end{tabular}

S30 


\begin{tabular}{|c|c|c|c|}
\hline $\mathrm{C}$ & 0.87202400 & 5.39346400 & 1.55722100 \\
\hline $\mathrm{C}$ & -0.04332800 & 6.17994800 & 0.82157800 \\
\hline $\mathrm{C}$ & -0.14154200 & 6.13625900 & -0.67928900 \\
\hline $\mathrm{C}$ & -1.04417800 & 5.30318100 & -1.37976600 \\
\hline $\mathrm{C}$ & -1.95855500 & 4.30944700 & -0.74289000 \\
\hline O & -1.96391700 & 4.28614000 & 0.57856400 \\
\hline $\mathrm{H}$ & -2.62361800 & 3.60038200 & 0.90347800 \\
\hline O & -2.67274600 & 3.56736200 & -1.42688500 \\
\hline $\mathrm{C}$ & -1.11733400 & 5.36441800 & -2.78097900 \\
\hline $\mathrm{C}$ & -0.32087600 & 6.24677800 & -3.49385500 \\
\hline $\mathrm{C}$ & 0.56685100 & 7.07226700 & -2.80528200 \\
\hline $\mathrm{C}$ & 0.67292500 & 7.02993200 & -1.41266300 \\
\hline $\mathrm{C}$ & 1.68136200 & 7.91774200 & -0.71837600 \\
\hline $\mathrm{H}$ & 2.47414800 & 7.31998300 & -0.25331400 \\
\hline $\mathrm{H}$ & 1.22760800 & 8.51909000 & 0.07699200 \\
\hline $\mathrm{H}$ & 2.15289200 & 8.59997400 & -1.43232000 \\
\hline $\mathrm{H}$ & 1.19921500 & 7.76308100 & -3.35819600 \\
\hline $\mathrm{H}$ & -0.38599100 & 6.29284400 & -4.57720100 \\
\hline $\mathrm{H}$ & -1.81360800 & 4.70386000 & -3.28441100 \\
\hline $\mathrm{C}$ & -0.87323200 & 7.09119700 & 1.51133800 \\
\hline $\mathrm{C}$ & -0.76756100 & 7.19696700 & 2.90158100 \\
\hline $\mathrm{C}$ & 0.13323200 & 6.41776200 & 3.62517300 \\
\hline $\mathrm{C}$ & 0.94639800 & 5.51592600 & 2.95398500 \\
\hline $\mathrm{H}$ & 1.65011700 & 4.89666500 & 3.49654000 \\
\hline $\mathrm{H}$ & 0.19712500 & 6.51375300 & 4.70530500 \\
\hline $\mathrm{H}$ & -1.41083500 & 7.90139800 & 3.42388100 \\
\hline $\mathrm{C}$ & -1.89347100 & 7.93239100 & 0.77754100 \\
\hline $\mathrm{H}$ & -2.67599100 & 7.30488300 & 0.33479000 \\
\hline $\mathrm{H}$ & -1.44557600 & 8.50554900 & -0.04164500 \\
\hline $\mathrm{H}$ & -2.37712800 & 8.63717300 & 1.46067200 \\
\hline O & -4.41281300 & 1.75694300 & -0.50788800 \\
\hline $\mathrm{C}$ & -4.45914100 & 1.77185300 & 0.81244400 \\
\hline O & -3.74757600 & 2.51429900 & 1.49851500 \\
\hline $\mathrm{C}$ & -5.44292000 & 0.84354100 & 1.44594600 \\
\hline $\mathrm{C}$ & -6.23104000 & -0.09861800 & 0.74568400 \\
\hline $\mathrm{C}$ & -7.11904700 & -0.92305900 & 1.47429700 \\
\hline $\mathrm{C}$ & -7.20129200 & -0.78670900 & 2.86299700 \\
\hline $\mathrm{C}$ & -6.42057600 & 0.14055300 & 3.55110600 \\
\hline $\mathrm{C}$ & -5.54304200 & 0.94677200 & 2.84261600 \\
\hline $\mathrm{H}$ & -4.91599200 & 1.67308700 & 3.34645900 \\
\hline $\mathrm{H}$ & -6.49676400 & 0.22864800 & 4.63112300 \\
\hline $\mathrm{H}$ & -7.88736200 & -1.42689600 & 3.41274400 \\
\hline $\mathrm{C}$ & -7.96023800 & -1.97175700 & 0.78121300 \\
\hline $\mathrm{H}$ & -7.33042300 & -2.75235700 & 0.33843400 \\
\hline $\mathrm{H}$ & -8.56111200 & -1.55024200 & -0.03208500 \\
\hline $\mathrm{H}$ & -8.63985800 & -2.45350200 & 1.49073300 \\
\hline $\mathrm{C}$ & -6.22485400 & -0.23984000 & -0.75248800 \\
\hline $\mathrm{C}$ & -5.39109400 & -1.14440200 & -1.44948800 \\
\hline $\mathrm{C}$ & -4.36189300 & -2.02026600 & -0.81312800 \\
\hline O & -4.32176700 & -2.00538000 & 0.50737100 \\
\hline $\mathrm{H}$ & -3.60898400 & -2.63602300 & 0.83326000 \\
\hline O & -3.60826000 & -2.72167800 & -1.49747300 \\
\hline C & -5.48323500 & -1.25448300 & -2.84621900 \\
\hline C & -6.39589900 & -0.49129900 & -3.55799200 \\
\hline C & -7.22011000 & 0.40014100 & -2.87327700 \\
\hline C & -7.14811300 & 0.54170500 & -1.48456100 \\
\hline C & -8.03884300 & 1.55108000 & -0.79492100 \\
\hline $\mathrm{H}$ & -7.44712100 & 2.36075600 & -0.35183700 \\
\hline $\mathrm{H}$ & -8.62157000 & 1.10318900 & 0.01750200 \\
\hline $\mathrm{H}$ & -8.73840400 & 1.99994800 & -1.50666300 \\
\hline
\end{tabular}

S31 


$\begin{array}{lrrr}\mathrm{H} & -7.93354400 & 1.00720500 & -3.42574000 \\ \mathrm{H} & -6.46542600 & -0.58460800 & -4.63802300 \\ \mathrm{H} & -4.82112300 & -1.95078300 & -3.34759100 \\ \mathrm{H} & -3.73761200 & 2.42844000 & -0.83219100\end{array}$

$(\mathrm{S}-1)_{4}$ conformer abab B3LYP/6-31G* optimized geometry [E=-3676.39939593 hartrees] Cartesian coordinates (Angstroms)

\begin{tabular}{|c|c|c|c|}
\hline O & 4.52288300 & 0.81060200 & 1.66331200 \\
\hline C & 4.56766200 & 1.78954000 & 0.76918800 \\
\hline $\mathrm{O}$ & 4.57645700 & 1.58259300 & -0.44740700 \\
\hline C & 4.62514200 & 3.15260900 & 1.36887000 \\
\hline C & 4.48779900 & 4.31624100 & 0.57875100 \\
\hline $\mathrm{C}$ & 4.55477400 & 5.58113900 & 1.20352700 \\
\hline C & 4.75898300 & 5.65221700 & 2.58517100 \\
\hline C & 4.88865500 & 4.50316400 & 3.36274300 \\
\hline C & 4.81605500 & 3.25742600 & 2.75569600 \\
\hline $\mathrm{H}$ & 4.90765300 & 2.35093600 & 3.34146300 \\
\hline $\mathrm{H}$ & 5.04320100 & 4.58139700 & 4.43513000 \\
\hline $\mathrm{H}$ & 4.81062100 & 6.63032600 & 3.05784400 \\
\hline $\mathrm{C}$ & 4.38223700 & 6.85818400 & 0.41189200 \\
\hline $\mathrm{H}$ & 3.36611100 & 6.93922900 & 0.00793800 \\
\hline $\mathrm{H}$ & 5.06660300 & 6.91179200 & -0.44186300 \\
\hline $\mathrm{H}$ & 4.55976400 & 7.73273200 & 1.04516600 \\
\hline C & 4.32058800 & 4.27890000 & -0.91613600 \\
\hline C & 3.06210100 & 4.28967400 & -1.56075600 \\
\hline C & 1.74323700 & 4.25498100 & -0.86107600 \\
\hline O & 0.68235700 & 4.26351600 & -1.49606300 \\
\hline $\mathrm{H}$ & -0.84911800 & 4.21907700 & -0.82821400 \\
\hline O & 1.78475300 & 4.22251800 & 0.45948100 \\
\hline C & 2.98912200 & 4.32393200 & -2.96318300 \\
\hline C & 4.14193200 & 4.35826500 & -3.73159500 \\
\hline C & 5.38406800 & 4.35122600 & -3.09815100 \\
\hline C & 5.49322300 & 4.30950500 & -1.70600400 \\
\hline C & 6.86440800 & 4.26116100 & -1.07059500 \\
\hline $\mathrm{H}$ & 7.02329500 & 3.30340400 & -0.56147500 \\
\hline $\mathrm{H}$ & 7.00439000 & 5.04832200 & -0.32154200 \\
\hline $\mathrm{H}$ & 7.64641800 & 4.37134500 & -1.82807600 \\
\hline $\mathrm{H}$ & 6.29317300 & 4.37183600 & -3.69473800 \\
\hline $\mathrm{H}$ & 4.07846100 & 4.38777000 & -4.81563000 \\
\hline $\mathrm{H}$ & 2.00752900 & 4.32332600 & -3.42248800 \\
\hline $\mathrm{H}$ & 4.52049100 & -0.07524300 & 1.19243300 \\
\hline O & 4.52288300 & -0.81060200 & -1.66331200 \\
\hline C & 4.56766200 & -1.78954000 & -0.76918800 \\
\hline O & 4.57645700 & -1.58259300 & 0.44740700 \\
\hline $\mathrm{C}$ & 4.62514200 & -3.15260900 & -1.36887000 \\
\hline C & 4.48779900 & -4.31624100 & -0.57875100 \\
\hline C & 4.55477400 & -5.58113900 & -1.20352700 \\
\hline $\mathrm{C}$ & 4.75898300 & -5.65221700 & -2.58517100 \\
\hline $\mathrm{C}$ & 4.88865500 & -4.50316400 & -3.36274300 \\
\hline $\mathrm{C}$ & 4.81605500 & -3.25742600 & -2.75569600 \\
\hline $\mathrm{H}$ & 4.90765300 & -2.35093600 & -3.34146300 \\
\hline $\mathrm{H}$ & 5.04320100 & -4.58139700 & -4.43513000 \\
\hline $\mathrm{H}$ & 4.81062100 & -6.63032600 & -3.05784400 \\
\hline C & 4.38223700 & -6.85818400 & -0.41189200 \\
\hline $\mathrm{H}$ & 3.36611100 & -6.93922900 & -0.00793800 \\
\hline $\mathrm{H}$ & 5.06660300 & -6.91179200 & 0.44186300 \\
\hline $\mathrm{H}$ & 4.55976400 & -7.73273200 & -1.04516600 \\
\hline
\end{tabular}




\begin{tabular}{|c|c|c|c|}
\hline C & 4.32058800 & -4.27890000 & 0.91613600 \\
\hline $\mathrm{C}$ & 3.06210100 & -4.28967400 & 1.56075600 \\
\hline C & 1.74323700 & -4.25498100 & 0.86107600 \\
\hline O & 0.68235700 & -4.26351600 & 1.49606300 \\
\hline $\mathrm{H}$ & -0.84911800 & -4.21907700 & 0.82821400 \\
\hline O & 1.78475300 & -4.22251800 & -0.45948100 \\
\hline C & 2.98912200 & -4.32393200 & 2.96318300 \\
\hline C & 4.14193200 & -4.35826500 & 3.73159500 \\
\hline C & 5.38406800 & -4.35122600 & 3.09815100 \\
\hline C & 5.49322300 & -4.30950500 & 1.70600400 \\
\hline $\mathrm{C}$ & 6.86440800 & -4.26116100 & 1.07059500 \\
\hline $\mathrm{H}$ & 7.02329500 & -3.30340400 & 0.56147500 \\
\hline $\mathrm{H}$ & 7.00439000 & -5.04832200 & 0.32154200 \\
\hline $\mathrm{H}$ & 7.64641800 & -4.37134500 & 1.82807600 \\
\hline $\mathrm{H}$ & 6.29317300 & -4.37183600 & 3.69473800 \\
\hline $\mathrm{H}$ & 4.07846100 & -4.38777000 & 4.81563000 \\
\hline $\mathrm{H}$ & 2.00752900 & -4.32332600 & 3.42248800 \\
\hline $\mathrm{H}$ & 4.52049100 & 0.07524300 & -1.19243300 \\
\hline O & -1.78475300 & 4.22251800 & -0.45948100 \\
\hline $\mathrm{C}$ & -1.74323700 & 4.25498100 & 0.86107600 \\
\hline O & -0.68235700 & 4.26351600 & 1.49606300 \\
\hline $\mathrm{H}$ & 0.84911800 & 4.21907700 & 0.82821400 \\
\hline $\mathrm{C}$ & -3.06210100 & 4.28967400 & 1.56075600 \\
\hline $\mathrm{C}$ & -4.32058800 & 4.27890000 & 0.91613600 \\
\hline $\mathrm{C}$ & -4.48779900 & 4.31624100 & -0.57875100 \\
\hline $\mathrm{C}$ & -4.62514200 & 3.15260900 & -1.36887000 \\
\hline $\mathrm{C}$ & -4.56766200 & 1.78954000 & -0.76918800 \\
\hline O & -4.52288300 & 0.81060200 & -1.66331200 \\
\hline $\mathrm{H}$ & -4.52049100 & -0.07524300 & -1.19243300 \\
\hline O & -4.57645700 & 1.58259300 & 0.44740700 \\
\hline $\mathrm{C}$ & -4.81605500 & 3.25742600 & -2.75569600 \\
\hline $\mathrm{C}$ & -4.88865500 & 4.50316400 & -3.36274300 \\
\hline $\mathrm{C}$ & -4.75898300 & 5.65221700 & -2.58517100 \\
\hline $\mathrm{C}$ & -4.55477400 & 5.58113900 & -1.20352700 \\
\hline $\mathrm{C}$ & -4.38223700 & 6.85818400 & -0.41189200 \\
\hline $\mathrm{H}$ & -3.36611100 & 6.93922900 & -0.00793800 \\
\hline $\mathrm{H}$ & -5.06660300 & 6.91179200 & 0.44186300 \\
\hline $\mathrm{H}$ & -4.55976400 & 7.73273200 & -1.04516600 \\
\hline $\mathrm{H}$ & -4.81062100 & 6.63032600 & -3.05784400 \\
\hline $\mathrm{H}$ & -5.04320100 & 4.58139700 & -4.43513000 \\
\hline $\mathrm{H}$ & -4.90765300 & 2.35093600 & -3.34146300 \\
\hline $\mathrm{C}$ & -5.49322300 & 4.30950500 & 1.70600400 \\
\hline $\mathrm{C}$ & -5.38406800 & 4.35122600 & 3.09815100 \\
\hline $\mathrm{C}$ & -4.14193200 & 4.35826500 & 3.73159500 \\
\hline $\mathrm{C}$ & -2.98912200 & 4.32393200 & 2.96318300 \\
\hline $\mathrm{H}$ & -2.00752900 & 4.32332600 & 3.42248800 \\
\hline $\mathrm{H}$ & -4.07846100 & 4.38777000 & 4.81563000 \\
\hline $\mathrm{H}$ & -6.29317300 & 4.37183600 & 3.69473800 \\
\hline $\mathrm{C}$ & -6.86440800 & 4.26116100 & 1.07059500 \\
\hline $\mathrm{H}$ & -7.02329500 & 3.30340400 & 0.56147500 \\
\hline $\mathrm{H}$ & -7.00439000 & 5.04832200 & 0.32154200 \\
\hline $\mathrm{H}$ & -7.64641800 & 4.37134500 & 1.82807600 \\
\hline $\mathrm{O}$ & -4.52288300 & -0.81060200 & 1.66331200 \\
\hline $\mathrm{C}$ & -4.56766200 & -1.78954000 & 0.76918800 \\
\hline $\mathrm{O}$ & -4.57645700 & -1.58259300 & -0.44740700 \\
\hline $\mathrm{C}$ & -4.62514200 & -3.15260900 & 1.36887000 \\
\hline $\mathrm{C}$ & -4.48779900 & -4.31624100 & 0.57875100 \\
\hline $\mathrm{C}$ & -4.55477400 & -5.58113900 & 1.20352700 \\
\hline $\mathrm{C}$ & -4.75898300 & -5.65221700 & 2.58517100 \\
\hline $\mathrm{C}$ & -4.88865500 & -4.50316400 & 3.36274300 \\
\hline
\end{tabular}




$\begin{array}{lrrr}\mathrm{C} & -4.81605500 & -3.25742600 & 2.75569600 \\ \mathrm{H} & -4.90765300 & -2.35093600 & 3.34146300 \\ \mathrm{H} & -5.04320100 & -4.58139700 & 4.43513000 \\ \mathrm{H} & -4.81062100 & -6.63032600 & 3.05784400 \\ \mathrm{C} & -4.38223700 & -6.85818400 & 0.41189200 \\ \mathrm{H} & -3.36611100 & -6.93922900 & 0.00793800 \\ \mathrm{H} & -5.06660300 & -6.91179200 & -0.44186300 \\ \mathrm{H} & -4.55976400 & -7.73273200 & 1.04516600 \\ \mathrm{C} & -4.32058800 & -4.27890000 & -0.91613600 \\ \mathrm{C} & -3.06210100 & -4.28967400 & -1.56075600 \\ \mathrm{C} & -1.74323700 & -4.25498100 & -0.86107600 \\ \mathrm{O} & -0.68235700 & -4.26351600 & -1.49606300 \\ \mathrm{H} & 0.84911800 & -4.21907700 & -0.82821400 \\ \mathrm{O} & -1.78475300 & -4.22251800 & 0.45948100 \\ \mathrm{C} & -2.98912200 & -4.32393200 & -2.96318300 \\ \mathrm{C} & -4.14193200 & -4.35826500 & -3.73159500 \\ \mathrm{C} & -5.38406800 & -4.35122600 & -3.09815100 \\ \mathrm{C} & -5.49322300 & -4.30950500 & -1.70600400 \\ \mathrm{C} & -6.86440800 & -4.26116100 & -1.07059500 \\ \mathrm{H} & -7.02329500 & -3.30340400 & -0.56147500 \\ \mathrm{H} & -7.00439000 & -5.04832200 & -0.32154200 \\ \mathrm{H} & -7.64641800 & -4.37134500 & -1.82807600 \\ \mathrm{H} & -6.29317300 & -4.37183600 & -3.69473800 \\ \mathrm{H} & -4.07846100 & -4.38777000 & -4.81563000 \\ \mathrm{H} & -2.00752900 & -4.32332600 & -3.42248800 \\ \mathrm{H} & -4.52049100 & 0.07524300 & 1.19243300\end{array}$

$(\mathrm{S}-1)_{4}$ conformer $\mathrm{abbb}$

B3LYP/6-31G* optimized geometry [E=-3676.39778119 hartrees] Cartesian coordinates (Angstroms)

$\begin{array}{lrll}\mathrm{O} & -1.85629700 & 4.01576500 & 1.77384900 \\ \mathrm{C} & -0.61932600 & 4.47902600 & 1.75371300 \\ \mathrm{O} & 0.00000000 & 4.68609300 & 0.70422700 \\ \mathrm{C} & 0.00000000 & 4.75893300 & 3.08426700 \\ \mathrm{C} & -0.60772700 & 4.48952100 & 4.33247300 \\ \mathrm{C} & 0.09778700 & 4.80092500 & 5.51755000 \\ \mathrm{C} & 1.37175600 & 5.36905500 & 5.43048600 \\ \mathrm{C} & 1.97004500 & 5.62991600 & 4.19879100 \\ \mathrm{C} & 1.28592300 & 5.32089900 & 3.03345400 \\ \mathrm{H} & 1.72413700 & 5.50417300 & 2.05933900 \\ \mathrm{H} & 2.96294300 & 6.06815300 & 4.15258600 \\ \mathrm{H} & 1.90511700 & 5.60336700 & 6.34885500 \\ \mathrm{C} & -0.48728200 & 4.49841700 & 6.87878200 \\ \mathrm{H} & -0.59697700 & 3.41763700 & 7.02656200 \\ \mathrm{H} & -1.48120900 & 4.93962300 & 7.01160500 \\ \mathrm{H} & 0.16222600 & 4.87996700 & 7.67246700 \\ \mathrm{C} & -2.00159600 & 3.94091400 & 4.47777500 \\ \mathrm{C} & -2.29202000 & 2.56190400 & 4.59141400 \\ \mathrm{C} & -1.27783600 & 1.46708900 & 4.53020700 \\ \mathrm{O} & -0.01203400 & 1.84665700 & 4.51418500 \\ \mathrm{H} & 0.58423400 & 1.03722100 & 4.49146400 \\ \mathrm{O} & -1.61984400 & 0.27941300 & 4.51573100 \\ \mathrm{C} & -3.61792300 & 2.13262200 & 4.76238900 \\ \mathrm{C} & -4.65469600 & 3.04970500 & 4.83948500 \\ \mathrm{C} & -4.37066200 & 4.41018700 & 4.73452700 \\ \mathrm{C} & -3.06386600 & 4.87117800 & 4.55056900 \\ \mathrm{C} & -2.81646200 & 6.35612400 & 4.40360200 \\ \mathrm{H} & -2.44341400 & 6.59678300 & 3.40121900\end{array}$




\begin{tabular}{|c|c|c|c|}
\hline $\mathrm{H}$ & -2.07059900 & 6.72495800 & 5.11647000 \\
\hline $\mathrm{H}$ & -3.74172000 & 6.91907000 & 4.55941700 \\
\hline $\mathrm{H}$ & -5.17945700 & 5.13522100 & 4.78865900 \\
\hline $\mathrm{H}$ & -5.67765800 & 2.71132900 & 4.97754600 \\
\hline $\mathrm{H}$ & -3.80431700 & 1.06726300 & 4.83195200 \\
\hline $\mathrm{H}$ & -2.18182400 & 3.87512200 & 0.83222600 \\
\hline O & -0.92014300 & 4.28891100 & -1.77973600 \\
\hline $\mathrm{C}$ & -2.17188300 & 3.86557300 & -1.75657500 \\
\hline O & -2.79042300 & 3.66753500 & -0.70451700 \\
\hline $\mathrm{C}$ & -2.81889300 & 3.64660900 & -3.08452900 \\
\hline $\mathrm{C}$ & -2.17594100 & 3.80261200 & -4.33422000 \\
\hline $\mathrm{C}$ & -2.91235400 & 3.55857400 & -5.51668000 \\
\hline $\mathrm{C}$ & -4.25202400 & 3.17247400 & -5.42488100 \\
\hline C & -4.88207300 & 3.01384200 & -4.19127500 \\
\hline $\mathrm{C}$ & -4.16429600 & 3.24665300 & -3.02888700 \\
\hline $\mathrm{H}$ & -4.62090700 & 3.12781300 & -2.05327800 \\
\hline $\mathrm{H}$ & -5.92355900 & 2.70908800 & -4.14188300 \\
\hline $\mathrm{H}$ & -4.80776300 & 2.98619500 & -6.34104800 \\
\hline $\mathrm{C}$ & -2.26568900 & 3.67304100 & -6.87869000 \\
\hline $\mathrm{H}$ & -1.49056000 & 2.90847500 & -7.00742300 \\
\hline $\mathrm{H}$ & -1.78365100 & 4.64498300 & -7.03086700 \\
\hline $\mathrm{H}$ & -3.00696400 & 3.53426200 & -7.67160200 \\
\hline $\mathrm{C}$ & -0.76005100 & 4.28930900 & -4.48469200 \\
\hline $\mathrm{C}$ & 0.34851300 & 3.41794700 & -4.57985300 \\
\hline C & 0.19071500 & 1.93842800 & -4.49333800 \\
\hline $\mathrm{O}$ & 1.34008500 & 1.27798300 & -4.43921900 \\
\hline $\mathrm{H}$ & 1.16176600 & 0.29094100 & -4.42471300 \\
\hline $\mathrm{O}$ & -0.90567400 & 1.37212400 & -4.48882100 \\
\hline $\mathrm{C}$ & 1.64129700 & 3.93524400 & -4.76055200 \\
\hline $\mathrm{C}$ & 1.84248000 & 5.30424300 & -4.86355900 \\
\hline $\mathrm{C}$ & 0.75023600 & 6.16520700 & -4.77602900 \\
\hline $\mathrm{C}$ & -0.54791400 & 5.68225300 & -4.58403400 \\
\hline $\mathrm{C}$ & -1.69364100 & 6.66133700 & -4.45894700 \\
\hline $\mathrm{H}$ & -2.12459800 & 6.63803700 & -3.45107800 \\
\hline $\mathrm{H}$ & -2.50776100 & 6.43711300 & -5.15687100 \\
\hline $\mathrm{H}$ & -1.35242300 & 7.68289900 & -4.65230400 \\
\hline $\mathrm{H}$ & 0.90421600 & 7.23912500 & -4.85162200 \\
\hline $\mathrm{H}$ & 2.84312100 & 5.70100100 & -5.00955200 \\
\hline $\mathrm{H}$ & 2.47631600 & 3.24801700 & -4.82104500 \\
\hline $\mathrm{H}$ & -0.58794500 & 4.41597800 & -0.83913600 \\
\hline O & 1.61984400 & -0.27941300 & 4.51573100 \\
\hline $\mathrm{C}$ & 1.27783600 & -1.46708900 & 4.53020700 \\
\hline O & 0.01203400 & -1.84665700 & 4.51418500 \\
\hline $\mathrm{H}$ & -0.58423400 & -1.03722100 & 4.49146400 \\
\hline $\mathrm{C}$ & 2.29202000 & -2.56190400 & 4.59141400 \\
\hline $\mathrm{C}$ & 2.00159600 & -3.94091400 & 4.47777500 \\
\hline $\mathrm{C}$ & 0.60772700 & -4.48952100 & 4.33247300 \\
\hline $\mathrm{C}$ & 0.00000000 & -4.75893300 & 3.08426700 \\
\hline C & 0.61932600 & -4.47902600 & 1.75371300 \\
\hline $\mathrm{O}$ & 1.85629700 & -4.01576500 & 1.77384900 \\
\hline $\mathrm{H}$ & 2.18182400 & -3.87512200 & 0.83222600 \\
\hline $\mathrm{O}$ & 0.00000000 & -4.68609300 & 0.70422700 \\
\hline $\mathrm{C}$ & -1.28592300 & -5.32089900 & 3.03345400 \\
\hline $\mathrm{C}$ & -1.97004500 & -5.62991600 & 4.19879100 \\
\hline $\mathrm{C}$ & -1.37175600 & -5.36905500 & 5.43048600 \\
\hline $\mathrm{C}$ & -0.09778700 & -4.80092500 & 5.51755000 \\
\hline $\mathrm{C}$ & 0.48728200 & -4.49841700 & 6.87878200 \\
\hline $\mathrm{H}$ & 0.59697700 & -3.41763700 & 7.02656200 \\
\hline $\mathrm{H}$ & 1.48120900 & -4.93962300 & 7.01160500 \\
\hline $\mathrm{H}$ & -0.16222600 & -4.87996700 & 7.6724670 \\
\hline
\end{tabular}




\begin{tabular}{|c|c|c|c|}
\hline $\mathrm{H}$ & -1.90511700 & -5.60336700 & 6.34885500 \\
\hline $\mathrm{H}$ & -2.96294300 & -6.06815300 & 4.15258600 \\
\hline $\mathrm{H}$ & -1.72413700 & -5.50417300 & 2.05933900 \\
\hline C & 3.06386600 & -4.87117800 & 4.55056900 \\
\hline C & 4.37066200 & -4.41018700 & 4.73452700 \\
\hline C & 4.65469600 & -3.04970500 & 4.83948500 \\
\hline C & 3.61792300 & -2.13262200 & 4.76238900 \\
\hline $\mathrm{H}$ & 3.80431700 & -1.06726300 & 4.83195200 \\
\hline $\mathrm{H}$ & 5.67765800 & -2.71132900 & 4.97754600 \\
\hline $\mathrm{H}$ & 5.17945700 & -5.13522100 & 4.78865900 \\
\hline C & 2.81646200 & -6.35612400 & 4.40360200 \\
\hline $\mathrm{H}$ & 2.44341400 & -6.59678300 & 3.40121900 \\
\hline $\mathrm{H}$ & 2.07059900 & -6.72495800 & 5.11647000 \\
\hline $\mathrm{H}$ & 3.74172000 & -6.91907000 & 4.55941700 \\
\hline $\mathrm{O}$ & 0.92014300 & -4.28891100 & -1.77973600 \\
\hline C & 2.17188300 & -3.86557300 & -1.75657500 \\
\hline $\mathrm{O}$ & 2.79042300 & -3.66753500 & -0.70451700 \\
\hline C & 2.81889300 & -3.64660900 & -3.08452900 \\
\hline C & 2.17594100 & -3.80261200 & -4.33422000 \\
\hline C & 2.91235400 & -3.55857400 & -5.51668000 \\
\hline C & 4.25202400 & -3.17247400 & -5.42488100 \\
\hline $\mathrm{C}$ & 4.88207300 & -3.01384200 & -4.19127500 \\
\hline C & 4.16429600 & -3.24665300 & -3.02888700 \\
\hline $\mathrm{H}$ & 4.62090700 & -3.12781300 & -2.05327800 \\
\hline $\mathrm{H}$ & 5.92355900 & -2.70908800 & -4.14188300 \\
\hline $\mathrm{H}$ & 4.80776300 & -2.98619500 & -6.34104800 \\
\hline C & 2.26568900 & -3.67304100 & -6.87869000 \\
\hline $\mathrm{H}$ & 1.49056000 & -2.90847500 & -7.00742300 \\
\hline $\mathrm{H}$ & 1.78365100 & -4.64498300 & -7.03086700 \\
\hline $\mathrm{H}$ & 3.00696400 & -3.53426200 & -7.67160200 \\
\hline C & 0.76005100 & -4.28930900 & -4.48469200 \\
\hline $\mathrm{C}$ & -0.34851300 & -3.41794700 & -4.57985300 \\
\hline $\mathrm{C}$ & -0.19071500 & -1.93842800 & -4.49333800 \\
\hline O & -1.34008500 & -1.27798300 & -4.43921900 \\
\hline $\mathrm{H}$ & -1.16176600 & -0.29094100 & -4.42471300 \\
\hline O & 0.90567400 & -1.37212400 & -4.48882100 \\
\hline C & -1.64129700 & -3.93524400 & -4.76055200 \\
\hline $\mathrm{C}$ & -1.84248000 & -5.30424300 & -4.86355900 \\
\hline $\mathrm{C}$ & -0.75023600 & -6.16520700 & -4.77602900 \\
\hline $\mathrm{C}$ & 0.54791400 & -5.68225300 & -4.58403400 \\
\hline $\mathrm{C}$ & 1.69364100 & -6.66133700 & -4.45894700 \\
\hline $\mathrm{H}$ & 2.12459800 & -6.63803700 & -3.45107800 \\
\hline $\mathrm{H}$ & 2.50776100 & -6.43711300 & -5.15687100 \\
\hline $\mathrm{H}$ & 1.35242300 & -7.68289900 & -4.65230400 \\
\hline $\mathrm{H}$ & -0.90421600 & -7.23912500 & -4.85162200 \\
\hline $\mathrm{H}$ & -2.84312100 & -5.70100100 & -5.00955200 \\
\hline $\mathrm{H}$ & -2.47631600 & -3.24801700 & -4.82104500 \\
\hline $\mathrm{H}$ & 0.58794500 & -4.41597800 & -0.83913600 \\
\hline
\end{tabular}

$(\mathrm{S}-1)_{4}$ conformer bbbb

B3LYP/6-31G* optimized geometry [E=-3676.39646655 hartrees] Cartesian coordinates (Angstroms)

$\begin{array}{ll}\mathrm{O} & -1.89298400 \\ \mathrm{C} & -1.90846200 \\ \mathrm{O} & -2.63313300 \\ \mathrm{C} & -1.00576600 \\ \mathrm{C} & -0.07703900 \\ \mathrm{C} & 0.72035700\end{array}$
4.38704700
4.41738600
3. 67977800
5.41740400
6.23043100
7. 13486600
0.52794400
$-0.79294000$
$-1.47043900$
$-1.43799700$
$-0.74838000$
$-1.48674300$ 


\begin{tabular}{|c|c|c|c|}
\hline $\mathrm{C}$ & 0.57219300 & 7.20728400 & -2.87469600 \\
\hline $\mathrm{C}$ & -0.34071300 & 6.40106600 & -3.55260200 \\
\hline C & -1.12103000 & 5.50812900 & -2.83443700 \\
\hline $\mathrm{H}$ & -1.83585000 & 4.86172300 & -3.33022300 \\
\hline $\mathrm{H}$ & -0.43805400 & 6.46984700 & -4.63231400 \\
\hline $\mathrm{H}$ & 1.19167700 & 7.90593800 & -3.43222600 \\
\hline $\mathrm{C}$ & 1.75369400 & 8.00355800 & -0.80467800 \\
\hline $\mathrm{H}$ & 2.55427600 & 7.39440300 & -0.36872800 \\
\hline $\mathrm{H}$ & 1.32458400 & 8.59455800 & 0.01185400 \\
\hline $\mathrm{H}$ & 2.21138600 & 8.69430200 & -1.51939300 \\
\hline $\mathrm{C}$ & 0.07703900 & 6.23043100 & 0.74838000 \\
\hline $\mathrm{C}$ & 1.00576600 & 5.41740400 & 1.43799700 \\
\hline $\mathrm{C}$ & 1.90846200 & 4.41738600 & 0.79294000 \\
\hline O & 1.89298400 & 4.38704700 & -0.52794400 \\
\hline $\mathrm{H}$ & 2.55067800 & 3.70279700 & -0.86079300 \\
\hline O & 2.63313300 & 3.67977800 & 1.47043900 \\
\hline $\mathrm{C}$ & 1.12103000 & 5.50812900 & 2.83443700 \\
\hline $\mathrm{C}$ & 0.34071300 & 6.40106600 & 3.55260200 \\
\hline $\mathrm{C}$ & -0.57219300 & 7.20728400 & 2.87469600 \\
\hline $\mathrm{C}$ & -0.72035700 & 7.13486600 & 1.48674300 \\
\hline $\mathrm{C}$ & -1.75369400 & 8.00355800 & 0.80467800 \\
\hline $\mathrm{H}$ & -2.55427600 & 7.39440300 & 0.36872800 \\
\hline $\mathrm{H}$ & -1.32458400 & 8.59455800 & -0.01185400 \\
\hline $\mathrm{H}$ & -2.21138600 & 8.69430200 & 1.51939300 \\
\hline $\mathrm{H}$ & -1.19167700 & 7.90593800 & 3.43222600 \\
\hline $\mathrm{H}$ & 0.43805400 & 6.46984700 & 4.63231400 \\
\hline $\mathrm{H}$ & 1.83585000 & 4.86172300 & 3.33022300 \\
\hline $\mathrm{H}$ & -2.55067800 & 3.70279700 & 0.86079300 \\
\hline O & -4.38704700 & 1.89298400 & -0.52794400 \\
\hline $\mathrm{C}$ & -4.41738600 & 1.90846200 & 0.79294000 \\
\hline O & -3.67977800 & 2.63313300 & 1.47043900 \\
\hline $\mathrm{C}$ & -5.41740400 & 1.00576600 & 1.43799700 \\
\hline $\mathrm{C}$ & -6.23043100 & 0.07703900 & 0.74838000 \\
\hline $\mathrm{C}$ & -7.13486600 & -0.72035700 & 1.48674300 \\
\hline $\mathrm{C}$ & -7.20728400 & -0.57219300 & 2.87469600 \\
\hline $\mathrm{C}$ & -6.40106600 & 0.34071300 & 3.55260200 \\
\hline $\mathrm{C}$ & -5.50812900 & 1.12103000 & 2.83443700 \\
\hline $\mathrm{H}$ & -4.86172300 & 1.83585000 & 3.33022300 \\
\hline $\mathrm{H}$ & -6.46984700 & 0.43805400 & 4.63231400 \\
\hline $\mathrm{H}$ & -7.90593800 & -1.19167700 & 3.43222600 \\
\hline $\mathrm{C}$ & -8.00355800 & -1.75369400 & 0.80467800 \\
\hline $\mathrm{H}$ & -7.39440300 & -2.55427600 & 0.36872800 \\
\hline $\mathrm{H}$ & -8.59455800 & -1.32458400 & -0.01185400 \\
\hline $\mathrm{H}$ & -8.69430200 & -2.21138600 & 1.51939300 \\
\hline $\mathrm{C}$ & -6.23043100 & -0.07703900 & -0.74838000 \\
\hline $\mathrm{C}$ & -5.41740400 & -1.00576600 & -1.43799700 \\
\hline $\mathrm{C}$ & -4.41738600 & -1.90846200 & -0.79294000 \\
\hline O & -4.38704700 & -1.89298400 & 0.52794400 \\
\hline $\mathrm{H}$ & -3.70279700 & -2.55067800 & 0.86079300 \\
\hline O & -3.67977800 & -2.63313300 & -1.47043900 \\
\hline $\mathrm{C}$ & -5.50812900 & -1.12103000 & -2.83443700 \\
\hline $\mathrm{C}$ & -6.40106600 & -0.34071300 & -3.55260200 \\
\hline $\mathrm{C}$ & -7.20728400 & 0.57219300 & -2.87469600 \\
\hline $\mathrm{C}$ & -7.13486600 & 0.72035700 & -1.48674300 \\
\hline $\mathrm{C}$ & -8.00355800 & 1.75369400 & -0.80467800 \\
\hline $\mathrm{H}$ & -7.39440300 & 2.55427600 & -0.36872800 \\
\hline $\mathrm{H}$ & -8.59455800 & 1.32458400 & 0.01185400 \\
\hline $\mathrm{H}$ & -8.69430200 & 2.21138600 & -1.51939300 \\
\hline $\mathrm{H}$ & -7.90593800 & 1.19167700 & -3.43222600 \\
\hline $\mathrm{H}$ & -6.46984700 & -0.43805400 & -4.63231400 \\
\hline
\end{tabular}




\begin{tabular}{|c|c|c|c|}
\hline $\mathrm{H}$ & -4.86172300 & -1.83585000 & -3.33022300 \\
\hline $\mathrm{H}$ & -3.70279700 & 2.55067800 & -0.86079300 \\
\hline O & 3.67977800 & 2.63313300 & -1.47043900 \\
\hline $\mathrm{C}$ & 4.41738600 & 1.90846200 & -0.79294000 \\
\hline O & 4.38704700 & 1.89298400 & 0.52794400 \\
\hline $\mathrm{H}$ & 3.70279700 & 2.55067800 & 0.86079300 \\
\hline $\mathrm{C}$ & 5.41740400 & 1.00576600 & -1.43799700 \\
\hline $\mathrm{C}$ & 6.23043100 & 0.07703900 & -0.74838000 \\
\hline $\mathrm{C}$ & 6.23043100 & -0.07703900 & 0.74838000 \\
\hline $\mathrm{C}$ & 5.41740400 & -1.00576600 & 1.43799700 \\
\hline $\mathrm{C}$ & 4.41738600 & -1.90846200 & 0.79294000 \\
\hline O & 4.38704700 & -1.89298400 & -0.52794400 \\
\hline $\mathrm{H}$ & 3.70279700 & -2.55067800 & -0.86079300 \\
\hline O & 3.67977800 & -2.63313300 & 1.47043900 \\
\hline $\mathrm{C}$ & 5.50812900 & -1.12103000 & 2.83443700 \\
\hline $\mathrm{C}$ & 6.40106600 & -0.34071300 & 3.55260200 \\
\hline $\mathrm{C}$ & 7.20728400 & 0.57219300 & 2.87469600 \\
\hline C & 7.13486600 & 0.72035700 & 1.48674300 \\
\hline $\mathrm{C}$ & 8.00355800 & 1.75369400 & 0.80467800 \\
\hline $\mathrm{H}$ & 7.39440300 & 2.55427600 & 0.36872800 \\
\hline $\mathrm{H}$ & 8.59455800 & 1.32458400 & -0.01185400 \\
\hline $\mathrm{H}$ & 8.69430200 & 2.21138600 & 1.51939300 \\
\hline $\mathrm{H}$ & 7.90593800 & 1.19167700 & 3.43222600 \\
\hline $\mathrm{H}$ & 6.46984700 & -0.43805400 & 4.63231400 \\
\hline $\mathrm{H}$ & 4.86172300 & -1.83585000 & 3.33022300 \\
\hline $\mathrm{C}$ & 7.13486600 & -0.72035700 & -1.48674300 \\
\hline $\mathrm{C}$ & 7.20728400 & -0.57219300 & -2.87469600 \\
\hline C & 6.40106600 & 0.34071300 & -3.55260200 \\
\hline $\mathrm{C}$ & 5.50812900 & 1.12103000 & -2.83443700 \\
\hline $\mathrm{H}$ & 4.86172300 & 1.83585000 & -3.33022300 \\
\hline $\mathrm{H}$ & 6.46984700 & 0.43805400 & -4.63231400 \\
\hline $\mathrm{H}$ & 7.90593800 & -1.19167700 & -3.43222600 \\
\hline $\mathrm{C}$ & 8.00355800 & -1.75369400 & -0.80467800 \\
\hline $\mathrm{H}$ & 7.39440300 & -2.55427600 & -0.36872800 \\
\hline $\mathrm{H}$ & 8.59455800 & -1.32458400 & 0.01185400 \\
\hline $\mathrm{H}$ & 8.69430200 & -2.21138600 & -1.51939300 \\
\hline O & 1.89298400 & -4.38704700 & 0.52794400 \\
\hline $\mathrm{C}$ & 1.90846200 & -4.41738600 & -0.79294000 \\
\hline O & 2.63313300 & -3.67977800 & -1.47043900 \\
\hline $\mathrm{C}$ & 1.00576600 & -5.41740400 & -1.43799700 \\
\hline $\mathrm{C}$ & 0.07703900 & -6.23043100 & -0.74838000 \\
\hline $\mathrm{C}$ & -0.72035700 & -7.13486600 & -1.48674300 \\
\hline $\mathrm{C}$ & -0.57219300 & -7.20728400 & -2.87469600 \\
\hline $\mathrm{C}$ & 0.34071300 & -6.40106600 & -3.55260200 \\
\hline $\mathrm{C}$ & 1.12103000 & -5.50812900 & -2.83443700 \\
\hline $\mathrm{H}$ & 1.83585000 & -4.86172300 & -3.33022300 \\
\hline $\mathrm{H}$ & 0.43805400 & -6.46984700 & -4.63231400 \\
\hline $\mathrm{H}$ & -1.19167700 & -7.90593800 & -3.43222600 \\
\hline C & -1.75369400 & -8.00355800 & -0.80467800 \\
\hline $\mathrm{H}$ & -2.55427600 & -7.39440300 & -0.36872800 \\
\hline $\mathrm{H}$ & -1.32458400 & -8.59455800 & 0.01185400 \\
\hline $\mathrm{H}$ & -2.21138600 & -8.69430200 & -1.51939300 \\
\hline $\mathrm{C}$ & -0.07703900 & -6.23043100 & 0.74838000 \\
\hline $\mathrm{C}$ & -1.00576600 & -5.41740400 & 1.43799700 \\
\hline $\mathrm{C}$ & -1.90846200 & -4.41738600 & 0.79294000 \\
\hline O & -1.89298400 & -4.38704700 & -0.52794400 \\
\hline $\mathrm{H}$ & -2.55067800 & -3.70279700 & -0.86079300 \\
\hline O & -2.63313300 & -3.67977800 & 1.47043900 \\
\hline $\mathrm{C}$ & -1.12103000 & -5.50812900 & 2.83443700 \\
\hline $\mathrm{C}$ & -0.34071300 & -6.40106600 & 3.5526020 \\
\hline
\end{tabular}

S38 


$\begin{array}{lrrr}\mathrm{C} & 0.57219300 & -7.20728400 & 2.87469600 \\ \mathrm{C} & 0.72035700 & -7.13486600 & 1.48674300 \\ \mathrm{C} & 1.75369400 & -8.00355800 & 0.80467800 \\ \mathrm{H} & 2.55427600 & -7.39440300 & 0.36872800 \\ \mathrm{H} & 1.32458400 & -8.59455800 & -0.01185400 \\ \mathrm{H} & 2.21138600 & -8.69430200 & 1.51939300 \\ \mathrm{H} & 1.19167700 & -7.90593800 & 3.43222600 \\ \mathrm{H} & -0.43805400 & -6.46984700 & 4.63231400 \\ \mathrm{H} & -1.83585000 & -4.86172300 & 3.33022300 \\ \mathrm{H} & 2.55067800 & -3.70279700 & 0.86079300\end{array}$




\section{METHODS}

S-(+)-2,2'-dimethylbiphenyl-6,6'-dicarboxylic acid, S-1, synthesized and characterized as described previously [1], was obtained from Dr. M. Tichý of the Institute of Organic Chemistry and Biochemistry of the Academy of Sciences of the Czech Republic. The optically pure sample exhibited $[\alpha]_{\mathrm{D}}=+21.7(\mathrm{c} 1, \mathrm{MeOH})$. Benzoic acid, 2, was obtained from Aldrich.

IR and VCD spectra of $\mathrm{CDCl}_{3}$ and DMSO- $\mathrm{d}_{6}$ solutions of S-1 were measured at room temperature using a Bruker IFS66/S FTIR spectrometer, equipped with a VCD/IRRAS module PMA 37 and a $\mathrm{CaF}_{2}$ demountable cell (Bruker, A145) [2]. Concentrations were in the range $0.001 \mathrm{M}-0.2 \mathrm{M}$. Pathlengths were in the range 50-1000 $\mu$. IR and VCD spectra were measured

at $4 \mathrm{~cm}^{-1}$ resolution. VCD scan times were $1.5 \mathrm{hr}$. Solvent IR and VCD spectra were used as the baselines for IR and VCD spectra of S-1. IR spectra of $\mathrm{CHCl}_{3}$ solutions of 2 were measured using a Nicolet MX-1 spectrometer. Concentrations were in the range 0.004-0.4 M. Pathlengths were in the range $45-1000 \mu$.

Vibrational frequencies, dipole strengths and rotational strengths were obtained from experimental IR and VCD spectra of S-1 assuming Lorentzian band shapes [5a,b] using the PeakFit (Version 4) software [Systat Software, Inc].

$A b$ initio DFT calculations were carried out using the programs GAUSSIAN 98 and GAUSSIAN 03 [3]. The B3LYP functional was used throughout together with the basis sets 6$31 \mathrm{G}^{*}, 6-311 \mathrm{G}^{* *}$ and $6-311++\mathrm{G}^{* *}$. Harmonic vibrational frequencies, dipole strengths and rotational strengths were obtained from Hessians (force fields), Atomic Polar Tensors (APTs) and Atomic Axial Tensors (AATs) [4]. AATs were calculated using Gauge-Invariant (Including) Atomic Orbitals (GIAOs); as a result, rotational strengths are origin-independent. IR and VCD spectra were obtained from vibrational frequencies, dipole strengths and rotational strengths assuming Lorentzian band shapes [4].

Experimental $\mathrm{A}$ and $\Delta \mathrm{A}$ values for $\mathrm{S}-\mathbf{1}$ are converted to $\varepsilon$ and $\Delta \varepsilon$ values using concentrations calculated assuming that $\mathrm{S}-\mathbf{1}$ is present as a monomer species; $\mathrm{D}$ and $\mathrm{R}$ values obtained thence reflect this choice. Calculated $\varepsilon$ and $\Delta \varepsilon$ values for (S-1) $)_{4}$ tetramers are obtained from calculated $\mathrm{D}$ and $\mathrm{R}$ values and then divided by 4 for consistency with the experimental spectra. Likewise, calculated $\mathrm{D}$ and $\mathrm{R}$ values are divided by 4 for comparison to experimental values. The IR spectrum of $\mathbf{2}$ is treated analogously. 


\section{REFERENCES}

1. Kanoh, S.; Muramoto, H.; Kobayashi, N.; Motoi, M.; Suda, H. Bull. Chem. Soc. Jpn 1987, 60, 3659-3662.

2. Urbanová, M.; Setnička, V.; Volka. K. Chirality 2000, 12, 199-203.

3. Frisch, M. J.; Trucks, G. W.; Schlegel, H. B.; Scuseria, G. E.; Robb, M. A.; Cheeseman, J. R.; Montgomery, Jr., J. A.; Vreven, T.; Kudin, K. N.; Burant, J. C.; Millam, J. M.; Iyengar, S. S.; Tomasi, J.; Barone, V.; Mennucci, B.; Cossi, M.; Scalmani, G.; Rega, N.; Petersson, G. A.; Nakatsuji, H.; Hada, M.; Ehara, M.; Toyota, K.; Fukuda, R.; Hasegawa, J.; Ishida, M.; Nakajima, T.; Honda, Y.; Kitao, O.; Nakai, H.; Klene, M.; Li, X.; Knox, J. E.; Hratchian, H. P.; Cross, J. B.; Bakken, V.; Adamo, C.; Jaramillo, J.; Gomperts, R.; Stratmann, R. E.; Yazyev, O.; Austin, A. J.; Cammi, R.; Pomelli, C.; Ochterski, J. W.; Ayala, P. Y.; Morokuma, K.; Voth, G. A.; Salvador, P.; Dannenberg, J. J.; Zakrzewski, V. G.; Dapprich, S.; Daniels, A. D.; Strain, M. C.; Farkas, O.; Malick, D. K.; Rabuck, A. D.; Raghavachari, K.; Foresman, J. B.; Ortiz, J. V.; Cui, Q.; Baboul, A. G.; Clifford, S.; Cioslowski, J.; Stefanov, B. B.; Liu, G.; Liashenko, A.; Piskorz, P.; Komaromi, I.; Martin, R. L.; Fox, D. J.; Keith, T.; Al-Laham, M. A.; Peng, C. Y.; Nanayakkara, A.; Challacombe, M.; Gill, P. M. W.; Johnson, B.; Chen, W.; Wong, M. W.; Gonzalez, C.; and Pople, J. A. Gaussian 98 and 03; Gaussian, Inc.: Pittsburgh, PA.

4. a) Devlin, F. J.; Stephens, P. J.; Cheeseman, J. R.; Frisch, M. J. J. Phys. Chem. 1997, 101, 6322-6333. (b) Devlin, F. J.; Stephens, P. J.; Cheeseman, J. R.; Frisch, M. J. J. Phys.

Chem. 1997, 101, 9912-9924. 